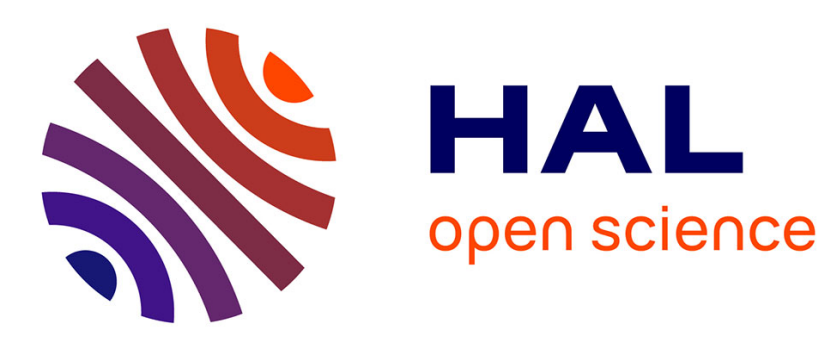

\title{
Witten non abelian localization for equivariant K-theory, and the $[\mathrm{Q}, \mathrm{R}]=0$ theorem
}

\author{
Paul-Emile Paradan, Michèle Vergne
}

\section{To cite this version:}

Paul-Emile Paradan, Michèle Vergne. Witten non abelian localization for equivariant K-theory, and the $[\mathrm{Q}, \mathrm{R}]=0$ theorem. Memoirs of the American Mathematical Society, In press, 10.1090/memo/1257 . hal-01145354v2

\section{HAL Id: hal-01145354 \\ https://hal.science/hal-01145354v2}

Submitted on 8 Jan 2016

HAL is a multi-disciplinary open access archive for the deposit and dissemination of scientific research documents, whether they are published or not. The documents may come from teaching and research institutions in France or abroad, or from public or private research centers.
L'archive ouverte pluridisciplinaire HAL, est destinée au dépôt et à la diffusion de documents scientifiques de niveau recherche, publiés ou non, émanant des établissements d'enseignement et de recherche français ou étrangers, des laboratoires publics ou privés. 


\section{Witten non abelian localization for equivariant $K$-theory, and the $[Q, R]=0$ theorem

\author{
Paul-Emile PARADAN* and Michèle VERGNE ${ }^{\dagger}$
}

January 8, 2016

\section{Contents}

1 Introduction 3

2 Notations $\quad 9$

3 Elliptic and transversally elliptic symbols $\quad 10$

4 Functoriality 14

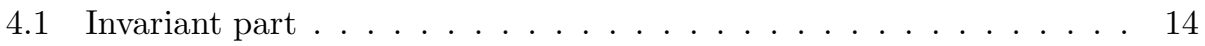

4.2 Free action . . . . . . . . . . . . . . . . . 15

4.3 Multiplicative property . . . . . . . . . . . . . . . . . 15

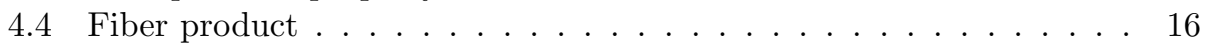

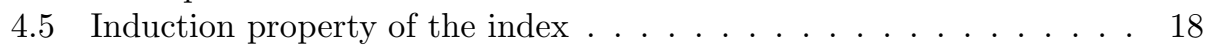

$5 \quad$ Clifford bundles and Dirac operators $\quad 18$

5.1 Clifford algebra . . . . . . . . . . . . . . . . . 18

5.2 Euclidean vector bundles and Clifford bundles . . . . . . . . . . . . 22

5.3 Choice of metrics . . . . . . . . . . . . . . . . . . . . . 23

5.4 The Bott symbol and direct image . . . . . . . . . . . . . . . 24

5.5 Dirac operators and equivariant indices _. . . . . . . . . 25

5.6 Horizontal Clifford bundle . . . . . . . . . . . . . . . . . . . 26

${ }^{*}$ Institut Montpelliérain Alexander Grothendieck, CNRS UMR 5149, Université de Montpellier, paul-emile.paradan@umontpellier.fr

${ }^{\dagger}$ Institut de Mathématiques de Jussieu, CNRS UMR 7586, Université Paris 7, vergne@math.jussieu.fr 
6 Deformation à la Witten of Dirac operators 27

6.1 Kirwan vector field . . . . . . . . . . . . . . . . . 28

6.2 Deforming a symbol . . . . . . . . . . . . . . . . . . . . . . . . . . . . . . . 31

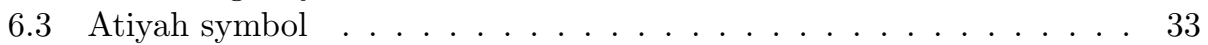

7 Abelian Localization formula $\quad 36$

7.1 Atiyah-Segal-Singer localization formula . . . . . . . . . . . . 36

7.2 Deformed Atiyah-Segal-Singer localization formula . . . . . . . . . . 38

8 Non abelian localization formula 39

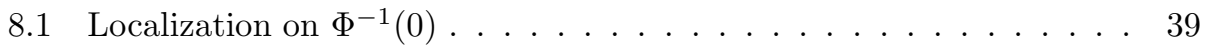

8.2 Coadjoint orbits and slices . . . . . . . . . . . . . . . . . . . . 41

8.3 Localization on $Z_{\beta}$. . . . . . . . . . . . . . . . . . . . . . . . . . . . 42

8.4 The non abelian localization theorem . . . . . . . . . . . . . . . 44

8.5 Example of $\mathrm{T}^{*} \tilde{K} \ldots \ldots \ldots \ldots \ldots$. . . . . . . . . . . . . . . . . . . . . . . .

$9[Q, R]=0$ Theorem $\quad 47$

9.1 A preliminary formula . . . . . . . . . . . . . . . 47

$9.2 \Phi$-positivity . . . . . . . . . . . . . . . . . . . . 49

$9.3[Q, R]=0$ in the asymptotic sense . . . . . . . . . . 51

$10[Q, R]=0$ for almost complex manifolds $\quad \mathbf{5 1}$

10.1 Statement of the results . . . . . . . . . . . . . . 52

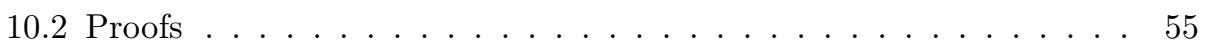

$10.3[Q, R]=0$ and semi-ample line bundles . . . . . . . . . 58

11 A slice theorem for deformed symbol 59

11.1 An induction formula . . . . . . . . . . . . . . . . 59

11.2 The shifting formula . . . . . . . . . . . . . . . . 61

12 The Hamiltonian setting $\quad 62$

$12.1[Q, R]=0$ Theorem of Meinrenken-Sjamaar . . . . . . . . . . 63

12.2 Localization on $\Phi^{-1}(0) \ldots \ldots \ldots$. . . . . . . . . . . . . . . . . . . . . . . . . . 65

12.2.1 Regular case .................... 65

12.2.2 Reduction in stage . . . . . . . . . . . . . . . 66

12.2.3 Quasi regular case ................ . . . 68

12.3 Riemann-Roch number on symplectic reduction . . . . . . . . . . . 75

12.4 Multiplicities as Riemann-Roch numbers . . . . . . . . . . . . . . 77

12.5 Quasi polynomial behavior . . . . . . . . . . . . . . 78

12.6 Multiplicities on a face . . . . . . . . . . . . . . . . 80

13 Branching laws $\quad 81$

13.1 Quasi polynomial behavior ................ . . . . . . 83

13.2 Multiplicities on a face . . . . . . . . . . . . . . 85 


\section{Introduction}

Let $M$ be a manifold provided with an action of a compact Lie group $K$ with Lie algebra $\mathfrak{k}$. Our main additional data is a $K$-invariant map $\Phi: M \rightarrow \mathfrak{k}^{*}$. This gives rise to a $K$-invariant vector field $\kappa_{\Phi}$, the Kirwan vector field, on $M$. In [50], Witten proposed a non abelian localization procedure on the zero set of the Kirwan vector field for the integration of equivariant classes in the Hamiltonian context. The $K$-theoretic analogue of the Witten non abelian localization procedure was introduced by the first author [32], still in the Hamiltonian context, in order to give a topological proof of the $[Q, R]=0$ theorem, after the first input of the second author in [48]. The $[Q, R]=0$ theorem, conjectured by Guillemin-Sternberg and proved by [29], is a remarkable result on the geometric quantization of symplectic manifolds, and we describe it below. Still with the aim of understanding the $[Q, R]=0$ theorem, this type of non abelian localization procedure has been transposed recently in the setting of equivariant $K$-homology by Y. Song [45]. The analytic approach of the Witten deformation has been studied by several people, see $[47,11,26,24,12,25]$.

The purpose of the present paper is three-fold.

First, we develop our K-theoretical analog of Witten deformation, when $M$ is any even dimensional oriented compact manifold, $\Phi$ a moment map in a weak sense, and $\sigma$ a symbol of any twisted Dirac operator. We use the vector field $\kappa_{\Phi}$ to deform an element $\sigma$ of the equivariant $\mathbf{K}$-theory of $\mathrm{T}^{*} M$. We obtain a general non abelian localization theorem, see Section 8.4, in equivariant $K$-theory. It localizes the study of the equivariant index of any $\sigma \in \mathbf{K}_{K}^{0}\left(\mathrm{~T}^{*} M\right)$ to the study of the equivariant index of the deformed element on a neighborhood of the zeroes of the map $\Phi$, together with the study of the same scenario on lower dimensional manifolds. In this deformation, an elliptic symbol is deformed in a sum of transversally elliptic symbols.

Our second purpose is to illustrate the power of this tool by reproving the $[Q, R]=0$ theorem of Meinrenken-Sjamaar [29] in a straightforward way. In other words, we clarify the strategy used by the first author in [32]. We also prove some generalizations, in the context of almost complex manifolds. In particular, we prove an asymptotic $[Q, R]=0$ theorem for Clifford bundles (Theorem 9.32) which plays an essential role when one studies the asymptotic behavior of branching law coefficients (see [37]) and the notion of stability.

As another application of our $K$-theoretical non-abelian localization theorem, we have obtained a geometric description of the multiplicities of the index of general twisted Dirac operators on general $K$-manifolds. This is 
the object of a separate article [40], as the proof requires further delicate information on admissible orbits, see [41].

Finally, our third purpose is to describe in detail some applications of the $[Q, R]=0$ theorem on multiplicities. If $M$ is a symplectic manifold and $L$ a Kostant line bundle on $M$, the geometric quantization $R R_{K}(M, L)$ of the symplectic manifold $M$ is a (virtual) representation space of $K$. We write $R R_{K}(M, L)=\sum_{\mu \in \hat{K}} \mathrm{~m}_{\mu}(L) V_{\mu}$. We give the proofs on the piecewise quasi-polynomial behavior of the multiplicity function $\mathrm{m}_{\mu}(L)$ as well as their "reduction behavior" on faces. All these results follow from understanding the geometric description of $\mathrm{m}_{\mu}(L)$ given by Meinrenken-Sjamaar, but this is sometimes not so easy to see while reading [29]. So we give a complete proof of all this facts here. We feel that it is worthwhile to explain a unified proof of these results, via Hamiltonian geometry and the $[Q, R]=0$ theorem. It seems that indeed these consequences were unnoticed, and other interesting proofs were given for interesting cases by several authors [22, 44, 14]. For example, the quasi polynomial behavior in $k$ of stretched LittlewoodRichardson coefficients [14], or of Kronecker coefficients [30], the reduction properties of these coefficients on faces [44], the factorization theorem of LR coefficients [20], the locally quasi-polynomial behavior of plethysm coefficients [18], are consequences of the geometric description of multiplicities obtained by Meinrenken-Sjamaar. Another interesting consequence of the asymptotic $[Q, R]=0$ theorem for Clifford bundles (Theorem 9.32) plays an essential role when one studies the asymptotic behavior of branching law coefficients. This is the object of another article by the first author [37].

Before describing our localization result, and its consequences, in more details, let us present some motivation and previous results. Let $A$ be a $K$ invariant elliptic operator on $M$ with principal symbol $\sigma$ and let $\operatorname{Index}_{K}^{M}(\sigma)(k)$ its index, a central function of $K$. Write $\operatorname{Index}_{K}^{M}(\sigma)(k)=\sum_{\mu \in \hat{K}} \mathrm{~m}(\mu, \sigma) \operatorname{Tr}_{\mu}(k)$ its Fourier expansion in terms of irreducible characters of $K$. The numbers $\mathrm{m}(\mu, \sigma) \in \mathbb{Z}$ are the multiplicities of the irreducible representation $V_{\mu}$ in the virtual space of solutions of $A$. In [16], Guillemin-Sternberg formulated the $[Q, R]=0$ principle (quantization commutes with reduction), which gave a conjectural geometric receipe to compute the multiplicities $\mathrm{m}\left(\mu, \sigma_{L}\right)$, in the case where $M$ is a Hamiltonian manifold equipped with a Kostant-Souriau line bundle $L$, in terms of the fibers of the moment map $\Phi: M \rightarrow \mathfrak{k}^{*}$ given by the Hamiltonian structure. Here $\sigma_{L}$ is the symbol of the Dolbeaut-Dirac operator with coefficients in $L$. Introduce the space $M_{\text {red }}=\Phi^{-1}(0) / K$ (and assume to simplify that $M_{\text {red }}$ is smooth). The space $M_{\text {red }}$ is the reduced Marsden-Weinstein symplectic space. It plays an important role, as for 
example many moduli spaces appear naturally as Marsden-Weinstein quotients. Also when $M$ is a projective manifold, the reduced manifold $M_{r e d}$ is the GIT quotient $M / / K_{\mathbb{C}}$ of $M$, where $K_{\mathbb{C}}$ is the complexification of $K$. Guillemin-Sternberg proved that the multiplicity of the trivial representation of $K$ is equal to the Riemann-Roch number of $M_{r e d}$, in the case where $M$ is a compact complex manifold and $L$ is ample. They conjectured that this equality is still valid in the Hamiltonian context [16]. Using symplectic cuts, Meinrenken-Sjamaar [29] gave the first proof of this fundamental theorem.

In view of describing cohomology rings of moduli spaces, Witten showed that integrals of cohomology classes on $M_{\text {red }}$ can be computed as the value at 0 of the Fourier transform of a function on $\mathfrak{k}$, obtained by integrating an equivariant cohomology class on the manifold $M$, using a deformation argument. This result suggested that a similar deformation argument could be used to study the Fourier expansion of equivariant indices. Indeed, in [32], the first author gave another proof of the $[Q, R]=0$ theorem, using deformation of the symbol $\sigma_{L}$. This proof is straightforward, once some functorial properties of the deformation are established. These functorial properties were established in [32], by several ad-hoc arguments. In this article, we prove these functorial properties in a general framework. We believe that this powerful technique can be useful to study equivariant indices in many different contexts. We see, for example, that a version of $[Q, R]=0$ theorem is true in this general context, in the asymptotic sense. Furthermore, we use the results of this article to prove a $[Q, R]=0$ exact principle in the context of Spin ${ }^{c}$-manifolds [40].

Let us now describe more precisely our context. Assume $M$ is even dimensional and consider a graded Clifford bundle $\mathcal{E} \rightarrow M$ for the tangent bundle $\mathrm{T} M$. We denote by $\mathbf{c}_{\mathcal{E}_{m}}$ the Clifford action of $\mathrm{T}_{m} M$ on the fiber $\mathcal{E}_{m}$. The symbol $\sigma(\mathcal{E})$ attached to the graded Clifford bundle is

$$
\sigma(\mathcal{E})(m, \nu)=\mathbf{c}_{\mathcal{E}_{m}}(\tilde{\nu}): \mathcal{E}_{m}^{+} \longrightarrow \mathcal{E}_{m}^{-}, \quad(m, \nu) \in \mathrm{T}^{*} M,
$$

where $\nu \in \mathrm{T}^{*} M \mapsto \tilde{\nu} \in \mathrm{T} M$ is an identification given by a $K$-invariant Riemannian structure on $M$. Note that such a symbol is invertible outside the zero section of $\mathrm{T}^{*} M$. Hence, when $M$ is compact, the symbols $\sigma(\mathcal{E})$ determines a class in the $\mathbf{K}$-group $\mathbf{K}_{K}^{0}\left(\mathrm{~T}^{*} M\right)$, and we denote $\mathcal{Q}_{K}(M, \mathcal{E})$ its equivariant index. If $K=\{1\}$ is the trivial group, $\mathcal{Q}_{K}(M, \mathcal{E}) \in \mathbb{Z}$ is an integer, denoted simply by $\mathcal{Q}(M, \mathcal{E})$.

We attach the Kirwan vector field $\kappa_{\Phi}$ to any equivariant map $\Phi: M \rightarrow$ $\mathfrak{k}^{*}:$ for $m \in M$, the vector $-\kappa_{\Phi}(m)$ is equal to the the tangent vector $\Phi(m) \cdot m$ 
associated to the infinitesimal action of $\Phi(m)$ on $m$. Here we have identified $\mathfrak{k}$ and $\mathfrak{k}^{*}$ by choosing a $K$-invariant inner product. We denote by $Z_{\Phi}$ the set of zeroes of $\kappa_{\Phi}$. Clearly $Z_{\Phi}$ contains $\Phi^{-1}(0)$. The deformed symbol $\sigma(\mathcal{E}, \Phi)$ is defined by

$$
\sigma(\mathcal{E}, \Phi)(m, \nu)=\mathbf{c}_{\mathcal{E}_{m}}(\tilde{\nu}+\Phi(m) \cdot m): \mathcal{E}_{m}^{+} \longrightarrow \mathcal{E}_{m}^{-} .
$$

The symbol $\sigma(\mathcal{E}, \Phi)$ can be restricted to a neighborhood of the set $Z_{\Phi}$ of zeroes of $\kappa_{\Phi}$ as a transversally elliptic symbol. If $Z$ is a union of connected components of $Z_{\Phi}$, we denote by $\sigma(\mathcal{E}, Z, \Phi)$ the restriction of $\sigma(\mathcal{E}, \Phi)$ to a small neighborhood of $Z$ and by $\mathcal{Q}_{K}(M, \mathcal{E}, Z, \Phi)$ the equivariant index of the symbol $\sigma(\mathcal{E}, Z, \Phi)$.

In this paper, an equivariant map $\Phi: M \rightarrow \mathfrak{k}^{*}$ is called ${ }^{1}$ a "moment map" if there is a closed two form $\Omega$ (possibly degenerate) such that the data $(\Omega, \Phi)$ satisfies the Kostant relations (6.14).

In Section 8, we prove the following non-abelian localization theorem (Theorem 8.6). When $\Phi$ is a moment map, the set $C_{\Phi}=\Phi\left(Z_{\Phi}\right)$ is a finite union of coadjoint orbits, and we define $Z_{\mathcal{O}}=Z_{\Phi} \cap \Phi^{-1}(\mathcal{O})$ for any $\mathcal{O} \subset C_{\Phi}$. We then have, for any $K$-equivariant graded Clifford bundle $\mathcal{E}$,

$$
\mathcal{Q}_{K}(M, \mathcal{E})=\sum_{\mathcal{O} \subset C_{\Phi}} \mathcal{Q}_{K}\left(M, \mathcal{E}, Z_{\mathcal{O}}, \Phi\right)
$$

When 0 is a regular value of $\Phi$, the character $\mathcal{Q}_{K}\left(M, \mathcal{E}, \Phi^{-1}(0), \Phi\right)$ can be described explicitly in terms of indices of elliptic symbols on the orbifold $M_{\text {red }}=\Phi^{-1}(0) / K$ (see Subsection 8.1). In particular we have that $\left[\mathcal{Q}_{K}\left(M, \mathcal{E}, \Phi^{-1}(0), \Phi\right)\right]^{K}=\mathcal{Q}\left(M_{\text {red }}, \mathcal{E}_{\text {red }}\right)$, where $\mathcal{E}_{\text {red }}$ is a graded Clifford bundle on $M_{\text {red }}$ induced by $\mathcal{E}$. In other words, the symbol $\sigma(\mathcal{E}, Z, \Phi)$ satisfies the $[Q, R]=0$ principle, for any Clifford bundle $\mathcal{E}$.

Let us describe the other components associated to $\mathcal{O}=K \beta$, with $\beta \neq 0$. Let $M^{\beta}$ be the set of zeroes of the vector field $\beta$, and $K_{\beta}$ the centralizer of $\beta$ in $K$ with Lie algebra $\mathfrak{k}_{\beta}$. The graded Clifford bundle $\mathcal{E}$ on $M$ induces a graded Clifford bundle $\mathbb{d}_{\beta}(\mathcal{E})$ on $M^{\beta}$. The action of $\beta$ provides a complex structure on the normal bundle to $M^{\beta}$, and we denote by $\mathcal{N}_{J_{\beta}}$ this complex vector bundle. Let $\operatorname{Sym}\left(\mathcal{N}_{J_{\beta}}\right)=\oplus_{k=0}^{\infty} \operatorname{Sym}^{k}\left(\mathcal{N}_{J_{\beta}}\right)$. Then $\mathcal{Q}_{K}\left(M, \mathcal{E}, Z_{\mathcal{O}}, \Phi\right)$ is the induced representation from $K_{\beta}$ to $K$ of the representation

$$
\mathcal{Q}_{K_{\beta}}\left(M^{\beta}, \mathbb{d}_{\beta}(\mathcal{E}) \otimes \operatorname{Sym}\left(\mathcal{N}_{J_{\beta}}\right), \Phi^{-1}(\beta),\left.\Phi\right|_{M^{\beta}}\right) \otimes \bigwedge\left(\mathfrak{k} / \mathfrak{k}_{\beta}\right)_{\mathbb{C}}
$$

\footnotetext{
${ }^{1}$ It corresponds to the notion of "abstract moment map" of Karshon.
} 
Here again, if $\beta$ is a regular value of the map $\Phi: M^{\beta} \rightarrow \mathfrak{k}_{\beta}^{*}$, this index can be explicitly described in terms of $\left(\Phi^{-1}(\beta) \cap M^{\beta}\right) / K_{\beta}$.

Starting in Section 9, we analyze the implications of the non-abelian localization theorem to the geometric study of the multiplicities. Let us remark that we do not assume before Section 12 that nor our group $K$ neither our manifold $M$ are connected. Indeed the study of the ring of invariants for classical groups such as $O(n)$ is an instance of the $[Q, R]=0$ theorem, and we want to include these cases.

We say that the $[Q, R]=0$ principle of Guillemin-Sternberg holds for the data $(\mathcal{E}, \Phi)$ if the multiplicity of the trivial representation in the equivariant index $\mathcal{Q}_{K}\left(M, \mathcal{E}, Z_{\mathcal{O}}, \Phi\right)$ vanishes if $\mathcal{O} \neq\{0\}$. Thanks to (1.1), this implies the identity

$$
\left[\mathcal{Q}_{K}(M, \mathcal{E})\right]^{K}=\left[\mathcal{Q}_{K}\left(M, \mathcal{E}, \Phi^{-1}(0), \Phi\right)\right]^{K},
$$

and this last space is described explicitly as $\mathcal{Q}\left(M_{\text {red }}, \mathcal{E}_{\text {red }}\right)$ when 0 is a regular value. If $\Phi$ is a moment map associated to an equivariant line bundle $L$, it is a straightforward consequence of our localization theorem that the $[Q, R]=0$ principle holds for the data $\left(\mathcal{E} \otimes L^{k}, \Phi\right)$, if $k$ is large enough (this result would also follow easily from the asymptotic analysis by Meinrenken in [27]). Consequences of this asymptotic result [37] includes many stability results on asymptotic behavior of branching coefficients.

We introduce a notion of $\Phi$-positivity for equivariant vector bundles and Clifford bundles which extends a similar notion introduced by Tian-Zhang in the Hamiltonian context [47].

When the Clifford bundle $\mathcal{E}$ is strictly $\Phi$-positive, we show that the $[Q, R]=0$ principle holds for the data $(\mathcal{E}, \Phi)$ : the multiplicity of trivial representation in the characters $\mathcal{Q}_{K}(M, \mathcal{E})$ and $\mathcal{Q}_{K}\left(M, \mathcal{E}, \Phi^{-1}(0), \Phi\right)$ are equal. If furthermore 0 is a regular value of $\Phi$, we get finally that

$$
\left[\mathcal{Q}_{K}(M, \mathcal{E})\right]^{K}=\mathcal{Q}\left(M_{\text {red }}, \mathcal{E}_{\text {red }}\right) .
$$

In Section 10, we specialize the study to the case of a $K$-manifold $M$ equipped with an invariant almost complex structure $J$. Then a natural Clifford bundle on $M$ is $\bigwedge_{J} \mathrm{~T} M$, and we consider the Riemann-Roch character

$$
R R_{K}^{J}(M, \mathcal{F}):=\mathcal{Q}_{K}\left(M, \bigwedge_{J} \mathrm{~T} M \otimes \mathcal{F}\right)
$$

that is attached to any equivariant vector bundle $\mathcal{F}$.

When $\Phi$ is a moment map associated to a closed 2 -form $\Omega$, we show that the Clifford bundle $\bigwedge_{J} \mathrm{~T} M$ is weakly $\Phi$-positive when the quadratic form 
$v \mapsto \Omega(v, J v)$ is non negative (we say that $J$ is adapted to $\Omega$ ). Hence the Clifford bundle $\mathcal{E}:=\bigwedge_{J} \mathrm{~T} M \otimes \mathcal{F}$ is strictly $\Phi$-positive when $J$ is adapted to $\Omega$, and the vector bundle $\mathcal{F}$ is strictly $\Phi$-positive.

An interesting example are the semi-ample line bundles $L$ on $(M, J)$ : we define those as line bundles $L$ for which there exists a $K$-invariant Hermitian connection $\nabla^{L}$ with curvature $\left(\nabla^{L}\right)^{2}=i \Omega_{L}$ such that $\Omega_{L}(v, J v)$ is semipositive definite. If $\Phi_{L}$ is the moment map attached to $\nabla^{L}$, we see then that the Clifford bundle $\bigwedge_{J} \mathrm{~T} M \otimes L$ is strictly $\Phi_{L}$-positive. In particular we get that $\left[R R_{K}^{J}\left(M, L^{\otimes k}\right)\right]^{K}=0$ for any $k \geq 1$ if $\Phi_{L}^{-1}(0)=\emptyset$.

In Section 12, we return to the case of a $K$-Hamiltonian manifold $(M, \Omega)$ with a Kostant-Souriau line bundle $L$ and moment map $\Phi$. Here we choose an almost complex structure $J$ that is compatible with the non degenerate 2 -form $\Omega$ : here the quadratic form $v \mapsto \Omega(v, J v)$ is positive definite. The corresponding quantized space $R R_{K}(M, L)=\sum_{\mu \in \hat{K}} \mathrm{~m}_{\mu}(L) V_{\mu}$ is a representation of $K$. As we have already seen in the case of semi-ample line bundles, the number $\mathrm{m}_{0}(L)=\left[R R_{K}(M, L)\right]^{K}$ is equal to

$$
\left[\mathcal{Q}_{K}\left(M, \bigwedge_{J} \mathrm{~T} M \otimes L, \Phi^{-1}(0), \Phi\right)\right]^{K} .
$$

If 0 is regular, we thus obtain that $\left[R R_{K}(M, L)\right]^{K}$ is the Riemann-Roch number $R R\left(M_{0}, L_{0}\right)$ of the orbifold bundle $L_{0}=\left.L\right|_{\Phi^{-1}(0)} / K$ on the reduced symplectic orbifold $M_{0}=\Phi^{-1}(0) / K$. Recall that this striking result was obtained by Meinrenken [28] using symplectic cutting. The core of Meinrenken-Sjamaar fundamental theorem is that (1.2) can be computed geometrically as the Riemann-Roch number of the (possibly singular) reduced space $M_{0}=\Phi^{-1}(0) / K$ :

- Either directly when 0 is a quasi-regular value of $\Phi$. The computation ${ }^{2}$, which is quite involved when 0 is quasi-regular, but not regular, is done in Section 12.2.3. In this case, the reduced space $M_{0}$ is again an orbifold.

- Or by moving the localization from $\Phi^{-1}(0)$ to the fiber of a quasiregular point close enough to 0 . This deformation argument is explained in Section 12.4.

Up to this point, our compact group $K$ could be not connected. As usual, for $K$ connected, one deals with the other multiplicities $\mathrm{m}_{\mu}(L)$ by using the shifting trick.

At the end of the paper, we describe the consequences of the $[Q, R]=0$ theorem on the piecewise locally polynomial behavior on the coefficients $\mathrm{m}_{\mu}(L)$, as well as their "reduction behavior" on faces.

\footnotetext{
${ }^{2}$ The quasi-regular case was not considered in [32].
} 


\section{Acknowledgments}

We wish to thank the Research in Pairs program at Mathematisches Forschungsinstitut Oberwolfach (February 2014), where this work was started. The second author wishes to thank Michel Duflo.

\section{Notations}

- Let $K$ be a compact (non necessarily connected) Lie group with Lie algebra $\mathfrak{k}$. We denote by $\hat{K}$ the set of isomorphism classes of irreducible complex finite dimensional representations of $K$. If $\mu \in \hat{K}$, we may also denote $\mu$ by $V_{\mu}$ (a vector space with an action of $K$ ). If $\mu \in \hat{K}$, we denote by $\mu^{*} \in \hat{K}$ the dual representation of $K$ in $V_{\mu^{*}}=V_{\mu}^{*}$.

Any finite dimensional representation $E$ of $K$ is isomorphic to a direct sum $E=\oplus_{\mu \in \widehat{K}} \mathrm{~m}(\mu) V_{\mu}$, and we say that $\mathrm{m}(\mu)$ is the multiplicity of $\mu$ in $E$. We denote by $[E]^{K}$ the subspace of $E$ formed by the $K$-fixed vectors. Thus $\mathrm{m}(\mu)=\operatorname{dim}\left[E \otimes V_{\mu}^{*}\right]^{K}$.

If $H$ is a subgroup of $K$, we denote by $\left.E\right|_{H}$ the space $E$ with the action of $H$.

- We denote by $R(K)$ the representation ring of $K:$ an element $E \in$ $R(K)$ can be represented as a finite sum $E=\sum_{\mu \in \widehat{K}} \mathrm{~m}(\mu) V_{\mu}$, with $\mathrm{m}(\mu) \in \mathbb{Z}$. We also say that $E$ is a virtual representation of $K$. The ring structure on $R(K)$ is induced by the tensor product and we denote by $\chi \otimes \mu$ the product. If $H$ is a subgroup of $K$, we denote by $\left.\chi \rightarrow \chi\right|_{H}$ the restriction $R(K) \rightarrow R(H)$.

We denote by $\hat{R}(K)$ the space of $\mathbb{Z}$-valued functions on $\hat{K}$. An element $E \in \hat{R}(K)$ can be represented as an infinite sum $E=\sum_{\mu \in \widehat{K}} \mathrm{~m}(\mu) V_{\mu}$, with $\mathrm{m}(\mu) \in \mathbb{Z}$. We say that $\mathrm{m}(\mu)$ is the multiplicity of $\mu$ in $E$. Then $[E]^{K} \in \mathbb{Z}$ and is the multiplicity of the trivial representation in $E$.

The space $\hat{R}(K)$ is a $R(K)$-module. If $H$ is a closed subgroup of $K$, the induction $\operatorname{map} \operatorname{Ind}_{H}^{K}: \hat{R}(H) \rightarrow \hat{R}(K)$ is the dual of the restriction morphism $R(K) \rightarrow R(H)$. If $\chi \in \hat{R}(H)$, then $\operatorname{Ind}_{H}^{K}(\chi)=\sum_{\lambda \in \hat{K}}\left[\left.\chi \otimes V_{\lambda}^{*}\right|_{H}\right]^{H} V_{\lambda}$. In particular $\left[\operatorname{Ind}_{H}^{K}(E)\right]^{K}=[E]^{H}$.

- We consider a positive definite $K$ invariant scalar product on $\mathfrak{k}$. This defines an isomorphism between $\mathfrak{k}$ and its dual vector space $\mathfrak{k}^{*}$.

- If $\mathfrak{s}$ is a subalgebra of $\mathfrak{k}$, we denote by $(\mathfrak{s})$ the conjugacy class of $\mathfrak{s}$ in the set of subalgebras of $\mathfrak{k}$. When $K$ acts on a set $\mathcal{X}$, the stabilizer subgroup of $x \in \mathcal{X}$ is denoted $K_{x}:=\{k \in K \mid k \cdot x=x\}$. The Lie algebra of $K_{x}$ is denoted by $\mathfrak{k}_{x}$. We denote by $\mathcal{X}_{\mathfrak{s}}$ the set of elements $x$ of $\mathcal{X}$ such $\mathfrak{k}_{x}=\mathfrak{s}$, and by $\mathcal{X}_{(\mathfrak{s})}=K \mathcal{X}_{\mathfrak{s}}$ the set of elements $x$ of $\mathcal{X}$ such $\mathfrak{k}_{x} \in(\mathfrak{s})$. 
When $K$ acts on a manifold $M$, we denote by $X_{M}(m):=\left.\frac{d}{d t}\right|_{t=0} e^{-t X} \cdot m$ the vector field generated by $-X \in \mathfrak{k}$. We may denote also $X_{M}(m)$ by $-X \cdot m$. We denote by $M^{X}$ the set of zeroes of the vector field $X_{M}$. This is a smooth manifold.

In particular, if $b \in \mathfrak{k}$ and $E$ is a representation space of $K$, then $E^{b}$ is the subspace of vectors in $E$ invariant by the one parameter subgroup exp $t b$ of $K$.

- When $V$ is a real vector space, we equip the direct sum $V \oplus V^{*}$ with the symplectic structure $\Omega$ defined by : $\Omega\left(v, v^{\prime}\right)=\Omega\left(f, f^{\prime}\right)=0$ and $\Omega(v, f)=$ $\langle f, v\rangle$, for $v, v^{\prime} \in V$ and $f, f^{\prime} \in V^{*}$. An Euclidean structure on $V$ induces a isomorphism $v \in V \mapsto \tilde{v} \in V^{*}$, and a complex structure $J_{V}$ on $V \oplus V^{*}$ defined by $J_{V} v=\tilde{v}$. Note that $\Omega\left(-, J_{V}-\right)$ is the scalar product on $V \oplus V^{*}$.

- Let $\mathcal{V} \rightarrow M$ be a vector bundle. An element $n$ of $\mathcal{V}$ may be written as $(m, v)$ with $m \in M,\left.v \in \mathcal{V}\right|_{m}$.

- The letter $E$ will denote a complex finite dimensional (eventually Hermitian) vector space, and $\mathcal{E} \rightarrow M$ a complex vector bundle. The space of smooth sections of $\mathcal{E}$ is denoted by $\Gamma(M, \mathcal{E})$.

Most of the times, the space $E$ will be $\mathbb{Z} / 2 \mathbb{Z}$ graded, $E=E^{+} \oplus E^{-}$. We may leave the $\mathbb{Z} / 2 \mathbb{Z}$ grading implicit. We denote by $E^{o p}$ the shift of parity on $E$ : we interchange $E^{+}$and $E^{-}$. Ungraded spaces $N$ will be considered as graded, with $N^{+}=N$ and $N^{-}=\{0\}$. Thus $(-1)^{k} E$ denotes the same graded vector space $E$, but where the change of parity has been performed $k$ times.

If $N$ is a complex (ungraded) vector space, then the exterior space $\bigwedge N$ will be $\mathbb{Z} / 2 \mathbb{Z}$ graded in even and odd elements. If $E=E^{+} \oplus E^{-}$and $F=F^{+} \oplus F^{-}$are two $\mathbb{Z} / 2 \mathbb{Z}$ graded Hermitian vector spaces, the tensor product $E \otimes F$ is $\mathbb{Z} / 2 \mathbb{Z}$-graded with $(E \otimes F)^{+}=E^{+} \otimes F^{+} \oplus E^{-} \otimes F^{-}$and $(E \otimes F)^{-}=E^{-} \otimes F^{+} \oplus E^{+} \otimes F^{-}$. If $f \in \operatorname{hom}\left(E^{+}, E^{-}\right), g \in \operatorname{hom}\left(F^{+}, F^{-}\right)$, we define $f \otimes g \in \operatorname{hom}\left((E \otimes F)^{+},(E \otimes F)^{-}\right)$by

$$
f \otimes g=\left(\begin{array}{cc}
f \otimes 1_{F_{+}} & -1_{E_{-}} \otimes g^{*} \\
1_{E_{+}} \otimes g & f^{*} \otimes 1_{F_{-}}
\end{array}\right) .
$$

- If $M$ is a $K$-manifold, and $E$ a representation space for $K$, we denote by $[E]$ the trivial vector bundle $M \times E$, with diagonal action of $K$.

\section{$3 \quad$ Elliptic and transversally elliptic symbols}

In this section, we recall the basic definitions from the theory of transversally elliptic symbols (or operators) defined by Atiyah-Singer in [1]. 
Let $M$ be a compact $K$-manifold with cotangent bundle $\mathrm{T}^{*} M$. Let $p: \mathrm{T}^{*} M \rightarrow M$ be the projection. If $\mathcal{E}$ is a vector bundle on $M$, we may denote still by $\mathcal{E}$ the vector bundle $p^{*} \mathcal{E}$ on the cotangent bundle $\mathrm{T}^{*} M$. If $\mathcal{E}^{+}, \mathcal{E}^{-}$are $K$-equivariant complex vector bundles over $M$, a $K$-equivariant morphism $\sigma \in \Gamma\left(\mathrm{T}^{*} M, \operatorname{hom}\left(\mathcal{E}^{+}, \mathcal{E}^{-}\right)\right)$is called a symbol on $M$. For $x \in M$, and $\nu \in T_{x}^{*} M$, thus $\sigma(x, \nu):\left.\left.\mathcal{E}\right|_{x} ^{+} \rightarrow \mathcal{E}\right|_{x} ^{-}$is a linear map. The subset of all $(x, \nu) \in \mathrm{T}^{*} M$ where the map $\sigma(x, \nu)$ is not invertible is called the characteristic set of $\sigma$, and is denoted by $\operatorname{Char}(\sigma)$. A symbol is elliptic if its characteristic set is compact.

Define the exterior product of symbols. Let $M_{1}, M_{2}$ be two manifolds with action of the compact groups $K_{1}, K_{2}$ and let $M=M_{1} \times M_{2}$. We write an element of $\mathrm{T}^{*} M=\mathrm{T}^{*} M_{1} \times \mathrm{T}^{*} M_{2}$ as $\left(\left(x_{1}, \nu_{1}\right),\left(x_{2}, \nu_{2}\right)\right)$. Let $f \in$ $\Gamma\left(\mathrm{T}^{*} M_{1}, \operatorname{hom}\left(\mathcal{E}^{+}, \mathcal{E}^{-}\right)\right)$and $g \in \Gamma\left(\mathrm{T}^{*} M_{2}, \operatorname{hom}\left(\mathcal{F}^{+}, \mathcal{F}^{-}\right)\right)$be two symbols. Using $K$-invariant Hermitian metrics on the bundles $\mathcal{E}^{ \pm}, \mathcal{F}^{ \pm}$we define the symbol $f \otimes g \in \Gamma\left(T^{*} M,(\mathcal{E} \otimes \mathcal{F})^{+},(\mathcal{E} \otimes \mathcal{F})^{-}\right)$by

$$
(f \otimes g)\left(\left(x_{1}, \nu_{1}\right),\left(x_{2}, \nu_{2}\right)\right)=f\left(x_{1}, \nu_{1}\right) \otimes g\left(x_{2}, \nu_{2}\right) .
$$

The symbol $f \otimes g$ is called the exterior product of the symbols $f, g$. Note that $\operatorname{Char}(f \otimes g)=\operatorname{Char}(f) \times \operatorname{Char}(g)$.

Consider now $M_{1}=M_{2}=M$ with an action of $K$. Let $f, g$ be two symbols on $M$. We still denote by $f \otimes g$ the restriction of the exterior product (a symbol on $M \times M$ ) to $M$ embedded as the diagonal in $M \times M$.

The product of a symbol $\sigma$ by a $K$-equivariant complex vector bundle $\mathcal{F} \rightarrow M$ is the symbol $\sigma \otimes \mathcal{F}$ defined by

$$
(\sigma \otimes \mathcal{F})(x, \nu)=\sigma(x, \nu) \otimes 1_{\mathcal{F}_{x}} .
$$

If $[E]$ is the vector bundle $M \times E$ associated to a representation space $E$ of $K$, we denote $\sigma \otimes[E]$ simply by $\sigma \otimes E$.

If $E=E^{+} \oplus E^{-}$is $\mathbb{Z} / 2 \mathbb{Z}$ graded, then

$$
\sigma \otimes E=\left(\begin{array}{cc}
\sigma \otimes 1_{E^{+}} & 0 \\
0 & \sigma^{*} \otimes 1_{E^{-}}
\end{array}\right) .
$$

An elliptic symbol $\sigma$ on $M$ defines an element $[\sigma]$ in the equivariant $\mathbf{K}$-theory of $\mathrm{T}^{*} M$ with compact support, which is denoted by $\mathbf{K}_{K}^{0}\left(\mathrm{~T}^{*} M\right)$. The class $\left[\sigma^{*}\right]$ is the inverse of $[\sigma]$ in $\mathbf{K}$-theory: $\left[\sigma^{*}\right]=-[\sigma]$. The index of $\sigma$ is a virtual finite dimensional representation of $K$, that we denote by $\operatorname{Index}_{K}^{M}(\sigma) \in R(K)[3,4,5,6]$. Recall its definition.

Let $A$ be a pseudo-differential operator $A: \Gamma\left(M, \mathcal{E}^{+}\right) \rightarrow \Gamma\left(M, \mathcal{E}^{-}\right)$from the space $\Gamma\left(M, \mathcal{E}^{+}\right)$of smooth sections of $\mathcal{E}^{+}$to the space $\Gamma\left(M, \mathcal{E}^{-}\right)$of 
smooth sections of $\mathcal{E}^{-}$, which commutes with the action of $K$. Here we follow the convention of [1][Lecture 1], so our pseudo-differential operator $A$ belongs to $\mathcal{P}^{m}\left(M, \mathcal{E}^{-}, \mathcal{E}^{+}\right)$and we can consider its principal symbol $\sigma_{p}$ which is defined outside the zero section of $\mathrm{T}^{*} M$. The operator $A$ is said to be elliptic if its principal symbol $\sigma_{p}(x, \nu)$ is invertible for all $(x, \nu) \in \mathrm{T}^{*} M$ such that $\nu \neq 0$. Then the space $\operatorname{ker}(A)$ of smooth solutions of $A$ is a finite dimensional representation space for $K$. Using a $K$-invariant function $\chi$ on $\mathrm{T}^{*} M$ identically equal to 1 in a neighborhood of $M$ and compactly supported, then $\sigma(x, \nu):=(1-\chi(x, \nu)) \sigma_{p}(x, \nu)$ is defined on the whole space $\mathrm{T}^{*} M$ and is an elliptic symbol. The class of $\sigma$ in $\mathbf{K}_{K}^{0}\left(\mathrm{~T}^{*} M\right)$ does not depend of the choice of $\chi$. We still say that this class $\sigma$ is the symbol of $A$. We choose $K$-invariant Hermitian structures on $\mathcal{E}^{+}, \mathcal{E}^{-}$. Then the formal adjoint $A^{*}: \Gamma\left(M, \mathcal{E}^{-}\right) \rightarrow \Gamma\left(M, \mathcal{E}^{+}\right)$is also an elliptic pseudodifferential operator. The index of $A$ is the virtual representation space $\operatorname{ker}(A)-\operatorname{ker}\left(A^{*}\right) \in R(K)$. This virtual representation depends only of the class of the symbol $\sigma$ of $A$ in $\mathbf{K}_{K}^{0}\left(\mathrm{~T}^{*} M\right)$, and by definition,

$$
\operatorname{Index}{ }_{K}^{M}(\sigma)=\operatorname{ker}(A)-\operatorname{ker}\left(A^{*}\right) .
$$

Recall the notion of transversally elliptic symbol. Let $\mathrm{T}_{K}^{*} M$ be the following $K$-invariant closed subset of $\mathrm{T}^{*} M$

$$
\mathrm{T}_{K}^{*} M=\left\{(x, \nu) \in \mathrm{T}^{*} M,\langle\nu, X \cdot x\rangle=0 \quad \text { for all } X \in \mathfrak{k}\right\} .
$$

Its fiber over a point $x \in M$ is formed by all the cotangent vectors $v \in$ $T_{x}^{*} M$ which vanish on the tangent space to the orbit of $x$ under $K$, in the point $x$. Thus each fiber $\left(\mathrm{T}_{K}^{*} M\right)_{x}$ is a linear subspace of $T_{x}^{*} M$. In general the dimension of $\left(\mathrm{T}_{K}^{*} M\right)_{x}$ is not constant and this space is not a vector bundle. A symbol $\sigma$ is $K$-transversally elliptic if the restriction of $\sigma$ to $\mathrm{T}_{K}^{*} M$ is invertible outside a compact subset of $\mathrm{T}_{K}^{*} M$ (i.e. $\operatorname{Char}(\sigma) \cap \mathrm{T}_{K}^{*} M$ is compact).

A $K$-transversally elliptic symbol $\sigma$ defines an element of $\mathbf{K}_{K}^{0}\left(\mathrm{~T}_{K}^{*} M\right)$, and the index of $\sigma$ defines an element $\operatorname{Index}_{K}^{M}(\sigma)$ of $\hat{R}(K)$. Recall its definition. A transversally elliptic operator $A: \Gamma\left(M, \mathcal{E}^{+}\right) \rightarrow \Gamma\left(M, \mathcal{E}^{-}\right)$is a pseudo-differential operator which is elliptic in the directions transversal to the orbits of $K$ : its principal symbol $\sigma_{p}(x, \nu)$ is invertible for all $(x, \nu) \in \mathrm{T}_{K}^{*} M$ such that $\nu \neq 0$. Thus, by restriction to $\mathrm{T}_{K}^{*} M$, the symbol $\sigma(x, \nu)=(1-\chi(x, \nu)) \sigma_{p}(x, \nu)$ defines a $K$-theory $\operatorname{class}^{3}[\sigma]$ in the topological equivariant $K$-theory group $\mathbf{K}_{K}^{0}\left(\mathrm{~T}_{K}^{*} M\right)$ which does not depend of

\footnotetext{
${ }^{3}$ To simplify the notations, we will also denote $\sigma \in \mathbf{K}_{K}^{0}\left(\mathrm{~T}_{K}^{*} M\right)$ the class defined by a transversally elliptic symbol $\sigma$.
} 
the choice of $\chi$. The index map associates to a transversally elliptic operator $A$ an element of $\hat{R}(K)$ constructed as follows. For every $\mu \in \hat{K}$, the space $\operatorname{hom}_{K}\left(V_{\mu}, \operatorname{ker}(A)\right)$ is a finite dimensional vector space of dimension $\mathrm{m}\left(V_{\mu}, A\right)$. Thus $\mathrm{m}\left(V_{\mu}, A\right)$ is the multiplicity of $V_{\mu}$ in the space $\operatorname{ker}(A)$ of smooth solutions of $A$. The number $\mathrm{m}\left(V_{\mu}, A\right)-\mathrm{m}\left(V_{\mu}, A^{*}\right)$ depends only of the class of the symbol $\sigma$ in $\mathbf{K}_{K}^{0}\left(\mathrm{~T}_{K}^{*} M\right)$ and by definition

$$
\operatorname{Index}_{K}^{M}(\sigma)=\sum_{\mu \in \hat{K}}\left(\mathrm{~m}\left(V_{\mu}, A\right)-\mathrm{m}\left(V_{\mu}, A^{*}\right)\right) V_{\mu} .
$$

In particular two symbols $\sigma_{1}$ and $\sigma_{2}$ having the same restriction to $\mathrm{T}_{K}^{*} M$ have the same index.

Remark 3.1 By definition, Index ${ }_{K}^{M}(\sigma)$ is isomorphic to a graded subspace of smooth (and $K$-finite) sections of $\mathcal{E}$, and we will not consider how to complete $\operatorname{Index}_{K}^{M}(\sigma)$ as an Hilbert space.

Example 3.2 A trivial, but important, example is the following. Consider $M=K$, with action of $K \times K$ by left and right translations, $\mathcal{E}^{+}=M \times \mathbb{C}$ the trivial vector bundle, $\mathcal{E}^{-}=M \times\{0\}$. Then $\Gamma\left(M, \mathcal{E}^{+}\right)=C^{\infty}(K), \Gamma\left(M, \mathcal{E}^{-}\right)=$ 0 , and the operator $A=0$ on $C^{\infty}(K)$ is transversally elliptic. Its symbol is the symbol $\sigma_{0}(k, \nu)=0$, and Peter-Weyl theorem shows that

$$
\operatorname{Index}_{K \times K}^{M}\left(\sigma_{0}\right)=\sum_{\mu \in \hat{K}} V_{\mu}^{*} \otimes V_{\mu} .
$$

The index map $\operatorname{Index}_{K}^{M}: \mathbf{K}_{K}^{0}\left(\mathrm{~T}_{K}^{*} M\right) \rightarrow \hat{R}(K)$ is a morphism of $R(K)$ module : for $E$ a representation space of $K$,

$$
\operatorname{Index}{ }_{K}^{M}(\sigma \otimes E)=\operatorname{Index}_{K}^{M}(\sigma) \otimes E .
$$

Any elliptic symbol is $K$-transversally elliptic, hence we have a restriction $\operatorname{map} \mathbf{K}_{K}^{0}\left(\mathrm{~T}^{*} M\right) \rightarrow \mathbf{K}_{K}^{0}\left(\mathrm{~T}_{K}^{*} M\right)$, and a commutative diagram

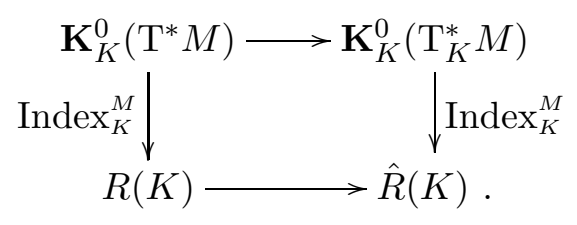

Let us explain how the index map Index ${ }_{K}^{M}$ is defined when $M$ is noncompact. Let $U$ be a non-compact $G$-manifold. Lemma 3.6 and Theorem 
3.7 of [1] tell us that for any open $K$-embedding $j: U \hookrightarrow N$ into a compact manifold we have a pushforward map $j_{*}: \mathbf{K}_{K}^{0}\left(\mathrm{~T}_{K}^{*} U\right) \rightarrow \mathbf{K}_{K}^{0}\left(\mathrm{~T}_{K}^{*} N\right)$ such that the composition

$$
\mathbf{K}_{K}^{0}\left(\mathrm{~T}_{K}^{*} U\right) \stackrel{j_{*}}{\longrightarrow} \mathbf{K}_{K}^{0}\left(\mathrm{~T}_{K}^{*} N\right) \stackrel{\text { Index }_{K}^{N}}{\longrightarrow} \hat{R}(K)
$$

is independant of the choice of $j: U \hookrightarrow N$ : this map is denoted $\operatorname{Index}_{K}^{U}$.

When $M$ is a non-compact manifold, the map $\operatorname{Index}_{K}^{M}: \mathbf{K}_{K}^{0}\left(\mathrm{~T}_{K}^{*} M\right) \rightarrow$ $\hat{R}(K)$ is defined as follows. Any class $\sigma \in \mathbf{K}_{K}^{0}\left(\mathrm{~T}_{K}^{*} M\right)$ is represented by a symbol on $M$ with a characteristic set $\operatorname{Char}(\sigma) \subset \mathrm{T}^{*} M$ intersecting $\mathrm{T}_{K}^{*} M$ in a compact set. Let $\mathcal{U}$ be a $K$-invariant relatively compact open subset of $M$ such that $\operatorname{Char}(\sigma) \cap \mathrm{T}_{K}^{*} M \subset \mathrm{T}^{*} \mathcal{U}$ : hence the restriction $\left.\sigma\right|_{\mathcal{U}}$ defines a class in $\mathbf{K}_{K}^{0}\left(\mathrm{~T}_{K}^{*} \mathcal{U}\right)$. The index map Index $\mathcal{U}_{K}^{\mathcal{H}}$ is well-defined since there exists an embedding $\mathcal{U} \hookrightarrow N$ into a compact $K$-manifold (see Lemma 3.1 in [32]). We take

$$
\operatorname{Index}_{K}^{M}(\sigma):=\operatorname{Index}_{K}^{\mathcal{U}}(\sigma \mid \mathcal{U}) .
$$

In particular, if $V$ is a vector space with a linear action of a compact group $K$, we can define the index of any $\sigma \in \mathbf{K}_{K}^{0}\left(\mathrm{~T}_{K}^{*} V\right)$.

Remark : In the following, the manifold $M$ will carry a $K$-invariant Riemannian metric and we will denote by $\nu \in \mathrm{T}^{*} M \mapsto \tilde{\nu} \in \mathrm{T} M$ the corresponding identification.

\section{Functoriality}

\subsection{Invariant part}

We will use the following obvious remark. Let $\sigma \in \Gamma\left(\mathrm{T}^{*} M, \operatorname{hom}\left(\mathcal{E}^{+}, \mathcal{E}^{-}\right)\right)$ be a $K$-transversally elliptic symbol on $M$. We assume that an invariant element $b \in \mathfrak{k}$ acts trivially on $M$. We can then define the subbundles $\left[\mathcal{E}^{ \pm}\right]^{b} \subset$ $\mathcal{E}^{ \pm}$and the $K$-transversally elliptic symbol $\sigma^{b} \in \Gamma\left(\mathrm{T}^{*} M, \operatorname{hom}\left(\left[\mathcal{E}^{+}\right]^{b},\left[\mathcal{E}^{-}\right]^{b}\right)\right)$ which is induced by $\sigma$.

Lemma 4.1 We have $\left[\operatorname{Index}_{K}^{M}(\sigma)\right]^{K}=\left[\operatorname{Index}_{K}^{M}\left(\sigma^{b}\right)\right]^{K}$. In particular $\left[\operatorname{Index}_{K}^{M}(\sigma)\right]^{K}=0$ if $\left[\mathcal{E}^{ \pm}\right]^{b}=0$.

Proof. We use a $K$-equivariant decomposition of vector bundles $\mathcal{E}^{ \pm}=$ $\left[\mathcal{E}^{ \pm}\right]^{b} \oplus \mathcal{F}^{ \pm}$. The symbol $\sigma$ is thus equal to $\sigma^{b} \oplus \sigma^{\prime}$, where the symbol $\sigma^{\prime} \in \Gamma\left(T^{*} M, \operatorname{hom}\left(\mathcal{F}^{+}, \mathcal{F}^{-}\right)\right)$is also $K$-transversally elliptic. The index map is additive so $\left[\operatorname{Index}_{K}^{M}(\sigma)\right]^{K}=\left[\operatorname{Index}_{K}^{M}\left(\sigma^{b}\right)\right]^{K}+\left[\operatorname{Index}_{K}^{M}\left(\sigma^{\prime}\right)\right]^{K}$. The space 
$\left[\operatorname{Index}_{K}^{M}\left(\sigma^{\prime}\right)\right]^{K}$ is constructed as the (virtual) subspace of invariant $C^{\infty}$ sections of the bundle $\mathcal{F}^{ \pm}$which are solutions of a $K$-invariant pseudodifferential operator on $M$ with symbol $\sigma^{\prime}$. But, as the action of $b$ is trivial on the basis, and $\left[\mathcal{F}^{ \pm}\right]^{b}=\{0\}$, the space of $b$-invariant $C^{\infty}$-sections of the bundle $\mathcal{F}^{ \pm}$is reduced to 0 . Hence $\left[\operatorname{Index}_{K}^{M}\left(\sigma^{\prime}\right)\right]^{K}=0$.

\subsection{Free action}

Let $G$ and $K$ be two compact Lie groups. Let $P$ be a manifold provided with an action of $G \times K$. We assume that the action of $K$ is free. Then the manifold $M:=P / K$ is provided with an action of $G$ and the quotient map $\pi: P \rightarrow M$ is a $G$-equivariant fibration. The action of $K$ on the bundle $\mathrm{T}_{K}^{*} P$ is free and the quotient $\left(\mathrm{T}_{K}^{*} P\right) / K$ admits a canonical identification with $\mathrm{T}^{*} M$. Then we still denote by

$$
q: \mathrm{T}_{K}^{*} P \rightarrow \mathrm{T}^{*} M
$$

the quotient map by $K$. The quotient map $q$ induces an isomorphism

$$
q^{*}: \mathbf{K}_{G}^{0}\left(\mathrm{~T}_{G}^{*} M\right) \rightarrow \mathbf{K}_{G \times K}^{0}\left(\mathrm{~T}_{G \times K}^{*} P\right)
$$

that makes $\mathbf{K}_{G}^{0}\left(\mathrm{~T}_{G}^{*} M\right)$ into a $R(K)$-module. For $V_{\mu}$ an irreducible representation of $K$, we denote by $\mathcal{V}_{\mu}$ the complex vector bundle $P \times_{K} V_{\mu}$ over $M$.

Theorem 4.2 (Free action property) We have the following equality in $\hat{R}(G \times K)$

$$
\operatorname{Index}_{G \times K}^{P}\left(q^{*} \sigma\right)=\sum_{\mu \in \hat{K}} \operatorname{Index}_{G}^{M}\left(\sigma \otimes \mathcal{V}_{\mu}^{*}\right) \otimes V_{\mu} .
$$

\subsection{Multiplicative property}

Let us recall the multiplicative property of the index for the product of manifolds. Consider a compact Lie group $G_{2}$ acting on two manifolds $M_{1}$ and $M_{2}$, and assume that another compact Lie group $G_{1}$ acts on $M_{1}$ commuting with the action of $G_{2}$. The external product of symbols induces a multiplication (see (2.3)):

$$
\otimes: \mathbf{K}_{G_{1} \times G_{2}}^{0}\left(\mathrm{~T}_{G_{1}}^{*} M_{1}\right) \times \mathbf{K}_{G_{2}}^{0}\left(\mathrm{~T}_{G_{2}}^{*} M_{2}\right) \longrightarrow \mathbf{K}_{G_{1} \times G_{2}}^{0}\left(\mathrm{~T}_{G_{1} \times G_{2}}^{*}\left(M_{1} \times M_{2}\right)\right) .
$$

Since $\mathrm{T}_{G_{1} \times G_{2}}^{*}\left(M_{1} \times M_{2}\right) \neq \mathrm{T}_{G_{1}}^{*} M_{1} \times \mathrm{T}_{G_{2}}^{*} M_{2}$ in general, the definition of the product $\left[\sigma_{1}\right] \otimes\left[\sigma_{2}\right]$ of transversally elliptic symbols needs some care: we have to take a representative $\sigma_{2}$ of $\left[\sigma_{2}\right]$ which is almost homogeneous. 
Theorem 4.3 (Multiplicative property) For any $\sigma_{1} \in \mathbf{K}_{G_{1} \times G_{2}}^{0}\left(\mathrm{~T}_{G_{1}}^{*} M_{1}\right)$ and any $\sigma_{2} \in \mathbf{K}_{G_{2}}^{0}\left(\mathrm{~T}_{G_{2}}^{*} M_{2}\right)$, we have

$$
\operatorname{Index}_{G_{1} \times G_{2}}^{M_{1} \times M_{2}}\left(\sigma_{1} \otimes \sigma_{2}\right)=\operatorname{Index}_{G_{1} \times G_{2}}^{M_{1}}\left(\sigma_{1}\right) \operatorname{Index}_{G_{2}}^{M_{2}}\left(\sigma_{2}\right) .
$$

In the last theorem, the product of $\operatorname{Index}_{G_{1} \times G_{2}}^{M_{1}}\left(\sigma_{1}\right) \in \hat{R}\left(G_{1} \times G_{2}\right)$ with Index $G_{2}^{M_{2}}\left(\sigma_{2}\right) \in \hat{R}\left(G_{2}\right)$ is well defined since $\operatorname{Index}_{G_{1} \times G_{2}}^{M_{1}}\left(\sigma_{1}\right)$ is smooth relatively to $G_{2}$.

\subsection{Fiber product}

Let $\mathcal{V} \rightarrow M$ be a $K$-equivariant Euclidean vector bundle.

Suppose that a central connected subgroup $\mathbb{\mathbb { }} \subset K$ acts trivially on $M$, so the torus $\mathbb{T}$ acts linearly on the fibers of $\mathcal{V}$. Let $V:=\left.\mathcal{V}\right|_{m_{o}}$ be a fiber which is equipped with a linear action of $\mathbb{T}$, and an $\mathbb{T}$-invariant scalar product. We denote by $O \subset O(V)$ the group formed by orthogonal endomorphisms of $V$ commuting with the $\mathbb{T}$-action. Let $P_{O} \rightarrow M$ be the $O$-principal bundle formed by the orthogonal linear maps $f:\left.V \rightarrow \mathcal{V}\right|_{m}$ commuting with the T-action. Then $P_{O}$ is a $K \times O$ manifold with free action of $O$.

Remark 4.4 If $\mathcal{V} \rightarrow M$ is a $K$-equivariant Hermitian vector bundle, we consider the group $U \subset U(V)$ formed by the unitary endomorphisms of $V$ commuting with the $\mathbb{T}$-action, and the $U$-principal bundle $P_{U} \rightarrow M$ formed by the unitary linear maps $f:\left.V \rightarrow \mathcal{V}\right|_{m}$ commuting with the $\mathbb{T}$-action.

We have two principal fibrations : $q_{M}: P_{O} \rightarrow M:=P_{O} / O$ and $q \mathcal{V}:$ $P_{O} \times V \rightarrow \mathcal{V}:=\left(P_{O} \times V\right) / O$. These quotient maps induce the following isomorphisms : $q_{M}^{*}: \mathbf{K}_{K}^{0}\left(\mathrm{~T}_{K}^{*} M\right) \rightarrow \mathbf{K}_{K \times O}^{0}\left(\mathrm{~T}_{K \times O}^{*} P_{O}\right)$ and $q_{\mathcal{V}}^{*}: \mathbf{K}_{K}^{0}\left(\mathrm{~T}_{K}^{*} \mathcal{V}\right) \rightarrow$ $\mathbf{K}_{K \times O}^{0}\left(\mathrm{~T}_{K \times O}^{*}\left(P_{O} \times V\right)\right)$.

Let us use the multiplicative property (see Section 4.3) with the groups $G_{2}=K \times O, G_{1}=\mathbb{T}$ acting on the manifolds $M_{1}=V, M_{2}=P_{O}$ (the subgroup $K$ of $G_{2}$ acting trivially on $V$ ).

We have a product

$$
\nabla: \mathbf{K}_{K \times O}^{0}\left(\mathrm{~T}_{K \times O}^{*} P_{O}\right) \times \mathbf{K}_{\mathbb{T} \times O}^{0}\left(\mathrm{~T}_{\mathbb{W}}^{*} V\right) \longrightarrow \mathbf{K}_{\mathbb{T} \times K \times O}^{0}\left(\mathrm{~T}_{\mathbb{T} \times K \times O}^{*}\left(P_{O} \times V\right)\right) .
$$

Let us consider the isomorphism

$$
q_{\mathcal{V}}^{*}: \mathbf{K}_{\mathbb{T} \times K}^{*}\left(\mathrm{~T}_{\mathbb{T} \times K}^{*} \mathcal{V}\right) \rightarrow \mathbf{K}_{\mathbb{T} \times K \times O}^{*}\left(\mathrm{~T}_{\mathbb{T} \times K \times O}^{*}\left(P_{O} \times V\right)\right) .
$$

Since the action of $\mathbb{\pi}$ on $\mathcal{V}$ comes from the $K$-action, we have $\mathrm{T}_{\mathbb{T} \times K}^{*} \mathcal{V}=$ $\mathrm{T}_{K}^{*} \mathcal{V}$, and then we can define a forgetful map $\mathrm{F}: \mathbf{K}_{\mathbb{T} \times K}^{0}\left(\mathrm{~T}_{K}^{*} \mathcal{V}\right) \rightarrow \mathbf{K}_{K}^{0}\left(\mathrm{~T}_{K}^{*} \mathcal{V}\right)$. 
Definition 4.5 The fiber product

$$
\diamond: \mathbf{K}_{K}^{0}\left(\mathrm{~T}_{K}^{*} M\right) \times \mathbf{K}_{\mathbb{T} \times O}^{0}\left(\mathrm{~T}_{\mathbb{T}}^{*} V\right) \longrightarrow \mathbf{K}_{K}^{0}\left(\mathrm{~T}_{K}^{*} \mathcal{V}\right)
$$

is defined by the relation

$$
\sigma \diamond \tau:=\mathrm{F} \circ\left(q_{\mathcal{V}}^{*}\right)^{-1}\left(q_{M}^{*}(\sigma) \otimes \tau\right) .
$$

A symbol representing $\sigma \diamond \tau$ can be constructed as follows. We write an element $n \in \mathcal{V}$ as $(x, v)$ with $x \in M$ and $\left.v \in \mathcal{N}\right|_{x}$. We choose a $K$ invariant connection on the bundle $\mathcal{N} \rightarrow M$, which allows us to parameterize $\mathrm{T}^{*} \mathcal{N}=\{(n,(\xi, \nu))\}$, with $\xi \in \mathrm{T}_{x}^{*} M,\left.\nu \in \mathcal{N}^{*}\right|_{x}$ (here $\xi$ is lifted to an element of $\mathrm{T}_{n}^{*} \mathcal{N}$ using the connection). Let $\sigma(x, \xi)$ be a symbol on $M$ representing $[\sigma]$ and $\tau(v, \eta)$ an almost homogeneous symbol on $V$ representing $\tau$. Then

$$
(\sigma \diamond \tau)((x, v),(\xi, \nu))=\sigma(x, \xi) \otimes \tau(v, \eta) .
$$

If $E$ is a representation space of the group $O$, we denote by $[[E]]$ the vector bundle $P_{O} \times_{O} E$ over $M$, and by $\sigma \otimes[[E]]$ the symbol $\sigma$ twisted by the vector bundle $[[E]]$.

Consider $\operatorname{Index}_{O}^{V}(\tau)$ as a representation space for $O$. As $\tau$ is $\mathbb{T}$-transversally elliptic, we have $\operatorname{Index}_{O}^{V}(\tau)=\sum_{\alpha \in \hat{\mathbb{V}}} E_{\alpha}$ where each $E_{\alpha}$ is a finite dimensional representation of $O$ where the action of $\mathbb{T}$ on $E_{\alpha}$ is scalar and acts via the character $\alpha$. We still denote by $\left[\left[\operatorname{Index}_{O}^{V}(\tau)\right]\right]$ the sum $\sum_{\alpha \in \hat{\mathbb{T}}}\left[\left[E_{\alpha}\right]\right]$ of finite dimensional $K$-vector bundles on $M$.

If we combine Theorems 4.2 and 4.3 , we get

Lemma 4.6 $\operatorname{Index}_{K}^{\mathcal{V}}(\sigma \diamond \tau)$ is equal to $\operatorname{Index}_{K}^{M}\left(\sigma \otimes\left[\left[\operatorname{Index}_{O}^{V}(\tau)\right]\right]\right)$ in $\hat{R}(K)$.

Let us explain briefly why $\operatorname{Index}_{K}^{M}\left(\sigma \otimes\left[\left[\operatorname{Index}_{O}^{V}(\tau)\right]\right]\right)$ is well defined in $\hat{R}(K)$. By definition $\operatorname{Index}_{K}^{M}\left(\sigma \otimes\left[\left[\operatorname{Index}_{O}^{V}(\tau)\right]\right]\right)$ is equal to the infinite sum

$$
\sum_{\alpha \in \hat{\mathbb{V}}} \operatorname{Index}_{K}^{M}\left(\sigma \otimes\left[\left[E_{\alpha}\right]\right]\right)
$$

Since $\mathbb{T}$ acts trivially on $M$, and commutes with $K$, it is not hard to see that for each $\mu \in \hat{K}$ the multiplicity of $V_{\mu}$ in $\operatorname{Index}_{K}^{M}\left(\sigma \otimes\left[\left[E_{\alpha}\right]\right]\right)$ is zero when $\|\alpha\|$ is large enough. This shows that the sum (4.8) converges in $\hat{R}(K)$.

An interesting case is already when the group $\mathbb{T}$ is trivial (see Section 5.4). Any elliptic element $\tau \in \mathbf{K}_{O(V)}^{0}\left(\mathrm{~T}^{*} V\right)$ defines a pushforward map $\mathbf{K}_{K}^{0}\left(\mathrm{~T}_{K}^{*} M\right) \rightarrow \mathbf{K}_{K}^{0}\left(\mathrm{~T}_{K}^{*} \mathcal{V}\right), \sigma \mapsto \sigma \diamond \tau$ such that $\operatorname{Index}_{K}^{\mathcal{V}}(\sigma \diamond \tau)=\operatorname{Index}_{K}^{M}(\sigma \otimes$ $[[\operatorname{Index} V(V)(\tau)]])$. 


\subsection{Induction property of the index}

Let $Y$ be a $H$-manifold and let $M:=K \times_{H} Y$. Since, at the level of the tangent spaces, we have the decomposition $\left.\mathrm{T} M\right|_{Y} \simeq \mathrm{T} Y \oplus Y \times \mathfrak{k} / \mathfrak{h}$, one sees that $\mathrm{T}_{K}^{*} M$ is diffeomorphic to $K \times_{H} \mathrm{~T}_{H}^{*} Y$. Hence we have an isomorphism $\mathbf{K}_{K}^{0}\left(\mathrm{~T}_{K}^{*} M\right) \simeq \mathbf{K}_{H}^{0}\left(\mathrm{~T}_{H}^{*} Y\right)$ : if $\sigma$ is a $K$-transversally elliptic symbol on $M$, we denoted $\left.\sigma\right|_{Y}$ the corresponding $H$-transversally elliptic symbol on $Y$.

Proposition 4.7 For any $K$-transversally elliptic symbol $\sigma$ on $M$

$$
\operatorname{Index}_{K}^{M}(\sigma)=\operatorname{Ind}_{H}^{K}\left(\operatorname{Index}_{H}^{Y}\left(\left.\sigma\right|_{Y}\right)\right) .
$$

Proof. Let us consider $N=K \times Y$ with action of $H$ on $K \times Y$ by $(k, y) \cdot h=\left(k h^{-1}, h y\right)$. Then $\mathrm{T}^{*} N=\mathrm{T}^{*} K \times \mathrm{T}^{*} Y$, and $\sigma$ defines a $K \times H$ transversally elliptic symbol $\tilde{\sigma}$ on $N$. The restriction of the symbol $\tilde{\sigma}$ to $\mathrm{T}_{K \times H}^{*} N=(K \times\{0\}) \times \mathrm{T}_{H}^{*} Y$ is equal to $\sigma_{0} \otimes \sigma_{Y}$, where $\sigma_{0}$ is the symbol of the zero operator on $C^{\infty}(K) \simeq \sum_{\lambda \in \hat{K}} V_{\lambda} \otimes V_{\lambda}^{*}$. Here we consider $C^{\infty}(K)$ with left action of $K$ and right action of $H$. Thus in $\hat{R}(K) \otimes \hat{R}(H)$, we have

$$
\operatorname{Index}_{K \times H}^{N}(\tilde{\sigma})=\operatorname{Index}_{K \times H}^{N}\left(\sigma_{0} \otimes \sigma_{Y}\right)=\sum_{\lambda \in \hat{K}} V_{\lambda} \otimes\left(\left.V_{\lambda}^{*}\right|_{H} \otimes \operatorname{Index}_{H}^{Y}\left(\left.\sigma\right|_{Y}\right)\right) .
$$

The index of $\sigma$ is obtained by taking the $H$-invariant part of $\operatorname{Index}_{K \times H}^{N}(\tilde{\sigma})$. We thus obtain the proposition.

Conversely, a $H$-equivariant symbol $\sigma_{Y}$ on $Y$ allows to define an induced $K$-equivariant symbol $\sigma$ on $K \times_{H} Y$ with restriction $\sigma_{Y}$, via $\sigma(y, q, \eta)=$ $\sigma(y, \eta)$ on $\mathrm{T}^{*} M \mid Y \simeq \mathfrak{q}^{*} \oplus T_{y}^{*} Y$. Then we have

$$
\operatorname{Index}_{K}^{M}(\sigma)=\operatorname{Ind}_{H}^{K}\left(\operatorname{Index}{ }_{H}^{Y}\left(\sigma_{Y}\right)\right) .
$$

\section{$5 \quad$ Clifford bundles and Dirac operators}

\subsection{Clifford algebra}

Let $V$ be an Euclidean space. We denote by $\mathrm{Cl}(V)$ its Clifford algebra. We follow the conventions of [8]. Denote by $(v, w)$ the scalar product of two vectors $v, w$ of $V$. Then, in $\mathrm{Cl}(V), v w+w v=-2(v, w)$. In particular $v^{2}=-\|v\|^{2}$ for any $v \in V \subset \mathrm{Cl}(V)$.

When $V$ is even dimensional and oriented, we define $\Gamma=i^{n / 2} e_{1} e_{2} \cdots e_{n}$ in $\mathrm{Cl}(V) \otimes \mathbb{C}$ modulo the choice of an oriented orthonormal basis $e_{1}, \ldots, e_{n}$. 
We remark that $\Gamma$ depends only of the orientation. Moreover we have $\Gamma^{2}=1$ and $\Gamma v=-v \Gamma$ for any $v \in V$.

If $E$ is a $\mathrm{Cl}(V)$-module, the Clifford map is denoted $\mathbf{c}_{E}: \mathrm{Cl}(V) \rightarrow$ $\operatorname{End}(E)$ : we have $\mathbf{c}_{E}(v)^{2}=-\|v\|^{2} \operatorname{Id}_{E}$ for any $v \in V$. A $\mathbb{Z} / 2 \mathbb{Z}$-graduation on $E$ is a vector space decomposition $E=E^{+} \oplus E^{-}$such that the map $\mathbf{c}_{E}(v): E \rightarrow E$ are odd maps for any $v \in V$. Equivalently, a grading on $E$ is defined by an involution $\theta: E \rightarrow E$ such that $\mathbf{c}_{E}(v) \circ \theta=-\mathbf{c}_{E}(v)$ for any $v \in V$.

For example, when $V$ is even dimensional and oriented, we may consider the $\mathbb{Z} / 2 \mathbb{Z}$-graduation on $E$ defined by the involution $\Gamma_{E}:=\mathbf{c}_{E}(\Gamma)$. This graduation will be called the canonical graduation of the $\mathrm{Cl}(V)$-module $E$.

Let us give the first example of Clifford module. Consider the real vector space $\bigwedge_{\mathbb{R}} V$. We denote by $m(v): \bigwedge_{\mathbb{R}} V \rightarrow \bigwedge_{\mathbb{R}} V$ the exterior multiplication by an element of $V$, and by $\iota(v): \bigwedge_{\mathbb{R}} V \rightarrow \bigwedge_{\mathbb{R}} V$ the contraction: $\iota(v)$ is the odd derivation of $\bigwedge_{\mathbb{R}} V$ such that $\iota\left(v_{2}\right) v_{1}=\left(v_{2}, v_{1}\right)$ for $v_{1} \in V \subset \bigwedge_{\mathbb{R}} V$. Then $\mathbf{c}(v)=m(v)-\iota(v)$ satisfies $\mathbf{c}(v)^{2}=-\|v\|^{2}$. Thus the complexified space $\left(\bigwedge_{\mathbb{R}} V\right) \otimes \mathbb{C} \simeq \bigwedge_{\mathbb{C}}(V \otimes \mathbb{C})$ is a Clifford module.

If $E$ is a graded Clifford module, and $W$ a graded complex vector space, the space $E \otimes W$ with Clifford action $\mathbf{c}_{E}(v) \otimes \operatorname{Id}_{W}$ is again a graded Clifford module where $(E \otimes W)^{+}=E^{+} \otimes W^{+} \bigoplus E^{-} \otimes W^{-}$and $(E \otimes W)^{-}=E^{-} \otimes$ $W^{+} \oplus E^{+} \otimes W^{-}$. It will be called the twisted Clifford module of $E$ with twisting space $W$.

If $E$ is a graded Clifford module, it is convenient to denote $-E$ the Clifford module with opposite grading. We can see $-E$ as the tensor of $E$ with the graded vector space $W$ where $W^{+}=\{0\}$ and $W^{-}=\mathbb{C}$.

Proposition 5.1 Let $V$ be an Euclidean space of even dimension $n=2 \ell$. There exists a $\mathrm{Cl}(V)$-module $S$ of such that the map $\mathbf{c}_{S}: \mathrm{Cl}(V) \rightarrow \operatorname{End}(S)$ extends to an isomorphism of complex algebras :

$$
\mathrm{Cl}(V) \otimes \mathbb{C} \simeq \operatorname{End}(S) .
$$

If an orientation of $V$ is given, we define $S^{+}=\{s \in S \mid \Gamma s=s\}$ and $S^{-}=\{s \in S \mid \Gamma s=-s\}$.

Then $S$ is unique, up to isomorphism. We call $S$ the spinor module. There is, up to a multiplicative constant, a unique Hermitian form on $S$ such that the operators $\mathbf{c}_{S}(v)$ are skew-adjoint.

For example if $V=\mathbb{R}^{2}$, with standard inner product, then $S$ is $\mathbb{C} \oplus \mathbb{C}$, with Clifford action

$$
\mathbf{c}\left(x e_{1}+y e_{2}\right)=\left(\begin{array}{cc}
0 & -(x-i y) \\
x+i y & 0
\end{array}\right) .
$$


Let $\mathrm{Spin}(\mathrm{V})$ be the spinor group. We denote by $\eta: \mathrm{Spin}(\mathrm{V}) \rightarrow \mathrm{SO}(\mathrm{V})$ the covering map. The kernel of $\tau$ has two elements. The spinor group $\operatorname{Spin}(\mathrm{V})$ acts in $S$, preserving $S^{+}, S^{-}$. We denote by $\rho$ the spin representation of Spin $(\mathrm{V})$. It satisfie $\rho(g) \mathbf{c}(v) \rho\left(g^{-1}\right)=\mathbf{c}(\eta(g) v)$.

Here is a construction of the spinor module. Consider $V \otimes \mathbb{C}$ and extend the form $($,$) by complex bilinearity to V \otimes \mathbb{C}$. Choose a decomposition $V \otimes \mathbb{C}=L_{1} \oplus L_{2}$ in two totally isotropic subspaces for the form (, ). Thus $L_{1}, L_{2}$ are of dimension $n / 2$. We consider on the $\mathbb{Z}_{2}$-graded complex vector space $\wedge L_{1}$ the following odd operators: the exterior multiplication $m\left(v_{1}\right)$ by an element of $L_{1}$, and the contraction $\iota\left(v_{2}\right)$ by an element of $L_{2}: \iota\left(v_{2}\right)$ is the odd derivation of $\bigwedge L_{1}$ such that $\iota\left(v_{2}\right) v_{1}=\left(v_{2}, v_{1}\right)$ for $v_{1} \in L_{1}$. The Clifford action of $V_{\mathbb{C}}$ on $\bigwedge L_{1}$ is defined by the formula

$$
\mathbf{c}\left(v_{1}+v_{2}\right)=\sqrt{2}\left(m\left(v_{1}\right)-\iota\left(v_{2}\right)\right) .
$$

Then $\mathbf{c}(v)$ is an odd operator on $\bigwedge L_{1}$ such that $\mathbf{c}(v)^{2}=-(v, v)$ Id. It is easy to see that $\bigwedge L_{1}$ is an irreducible Clifford module. Thus $\bigwedge L_{1}$ with action c $(v)$ as above is "the" spinor module.

Let $J$ be an endomorphism of $V$ such that $J^{2}=-1$ ( $J$ defines a complex structure on $V)$ and such that $(J v, J v)=(v, v)$. Equivalently $h(v, w)=$ $g(v, w)-i g(J v, w)$ is a Hermitian structure on the complex vector space $(V, J)$. Then $J$ defines also an orientation $o(J)$ of $V$. If $e_{1}, e_{2}, \ldots, e_{n / 2}$ is a basis of $V$ as a complex space, then we take as oriented basis of $V$ the basis $e_{1}, J e_{1}, e_{2}, J e_{2}, \ldots, e_{n / 2}, J e_{n / 2}$. We denote by $V_{J}$ the space $V$ considered as a complex vector space and we may also denote by $\bigwedge_{J} V=\bigwedge V_{J}$ the exterior space of the complex space $V_{J}$.

We denote by

$$
V^{+}=\{v \in V \otimes \mathbb{C} \mid J v=i v\}, \quad V^{-}=\{v \in V \otimes \mathbb{C} \mid J v=-i v\} .
$$

These are two totally isotropic spaces for $\left(\right.$, ) and we can take $S=\bigwedge V^{+}$as the irreducible Clifford module. In complex geometry, it is more customary to take $S=\bigwedge\left(V^{-}\right)^{*}$, but the space $\left(V^{-}\right)^{*}$ is isomorphic to $V^{+}$by the duality $g($,$) .$

The map $v \rightarrow v-i J v$ from $V$ to $V^{+}$gives an isomorphism of the complex vector spaces $V_{J}$ with $V^{+}$. Then $\bigwedge V_{J}$ is isomorphic to $\bigwedge V^{+}$, and we can realize $S$ as $\wedge V_{J}$. The grading of $S$ induced by the orientation $o(J)$ of $V_{J}$ is then $S^{+}=\bigwedge^{e v e n} V_{J}$ and $S^{-}=\bigwedge^{\text {odd }} V_{J}$.

If $H$ is a Hermitian vector space, in some situation (see Section 6.3), it is convient to choose as spinor space $\bigwedge \bar{H}$, where $\bar{H}$ denote the space $H$, with 
complex structure $-J$. Then the grading $\bigwedge^{\text {even }} \bar{H} \oplus \bigwedge^{\text {odd }} \bar{H}$ corresponds to the orientation $o(-J)=(-1)^{\operatorname{dim} H} o(J)$.

Consider the subgroup $U(V)$ of $S O(V)$ consisting of complex transformations of $(V, J)$. An element of $U(V)$ leaves stable the space $V^{+}$, and we obtain a natural action $\lambda$ of $U(V)$ in $\bigwedge V^{+}$. Let us compare the natural action $\lambda$ and the restriction $\rho$ of the spin representation of $\operatorname{Spin}(\mathrm{V})$ to $\tau^{-1}(U(V))$.

Lemma 5.2 For $g \in \tau^{-1}(U(V)) \subset \operatorname{Spin}(\mathrm{V}), \lambda(\tau(g))=\delta(g) \rho(g)$, where $\delta(g)$ is the character of $\tau^{-1}(U(V))$ such that $\delta(g)^{2}=\operatorname{det}_{V^{+}}(g)$.

We often will use complex structures on real vector spaces defined by the following procedure.

Definition 5.3 Let $N$ be a real vector space and $\beta$ a transformation of $N$, such that $-\beta^{2}$ is diagonalizable with non negative eigenvalues. Define

- the transformation $|\beta|$ of $N$ by $|\beta|=\sqrt{-\beta^{2}}$,

- and the complex structure $J_{\beta}$ on $N / \operatorname{ker}(\beta)$ by $J_{\beta}=\beta|\beta|^{-1}$.

For example if $V$ is an Euclidean space and $\beta$ a skew-symmetric transformation of $V$, then $-\beta^{2}$ is diagonalizable with non negative eigenvalues. By definition of $J_{\beta}$, the transformation $\beta$ induces on $N / \operatorname{ker}(\beta) \simeq \operatorname{Image}(\beta)$ a complex diagonalizable transformation, and the list of its complex eigenvalues is $\left[i a_{1}, \ldots, i a_{\ell}\right]$ where the $a_{k}$ are strictly positive real numbers.

Similarly if $\mathcal{N} \rightarrow B$ is a Euclidean vector bundle with a fibrewise bijective linear endomorphism $\mathcal{L}(\beta)$ which is anti-symmetric relatively to the metric, we denote by $\mathcal{N}_{J_{\beta}}$ the vector bundle $\mathcal{N}$ over $B$ equipped with the complex structure $J_{\beta}$. Then $\mathcal{N}_{J_{\beta}}$ is a Hermitian vector bundle over $B$, and we say that $\mathcal{N}_{J_{\beta}}$ is the complex vector bundle $\mathcal{N}$ polarized by $\beta$.

The following proposition follows from the Schur lemma. It is used to "divide" a Clifford module by the irreducible Clifford module $S$.

Proposition 5.4 When $\operatorname{dim} V$ is even, any $\mathrm{Cl}(V)$-module $E$ has the following decomposition

$$
E \simeq S \otimes \operatorname{hom}_{\mathrm{Cl}(V)}(S, E)
$$

where $\operatorname{hom}_{\mathrm{Cl}(V)}(S, E)$ is the vector space of $\mathrm{Cl}(V)$-complex linear maps from $S$ to $E$. The grading of $E$, and a grading on $S$ coming from an orientation on $V$ induces a grading on $\operatorname{hom}_{\mathrm{Cl}(V)}(S, E)$ so that the isomorphism (5.10) is an isomorphism of graded Clifford module. 
The space $W=\operatorname{hom}_{\mathrm{Cl}(V)}(S, E)$ is called the twisting space. We write $E=S \otimes W$ and the Clifford action is just on the spinor module $S$ :

$$
\mathbf{c}_{E}(v)=\mathbf{c}_{S}(v) \otimes \operatorname{Id}_{W} .
$$

Example 5.5 - If $J_{1}, J_{2}$ are two complex structures on $V$, we may compare the spinor bundle $S_{k}=\bigwedge V_{J_{k}}$. We have $S_{1} \simeq \epsilon S_{2}$ where $\epsilon \in\{ \pm 1\}$ is the ratio between the orientations $o\left(J_{1}\right)$ and $o\left(J_{2}\right)$.

- If $V$ is even dimensional, the twisting space $W$ for the Clifford module $\bigwedge_{\mathbb{R}} V \otimes \mathbb{C}$ is $-S$.

We implicitly always choose Hermitian products on $E$ obtained by tensor product of the Hermitian structure on $S$ and a Hermitian structure on $W$. Then the operators $\mathbf{c}(v)$ are skew-adjoint.

If $V=V_{1} \oplus V_{2}$ is an orthogonal decomposition, we have $\mathrm{Cl}(V) \simeq \mathrm{Cl}\left(V_{1}\right) \otimes$ $\mathrm{Cl}\left(V_{2}\right)$. So, if $E_{1}, E_{2}$ are graded Clifford modules for $V_{1}, V_{2}$, then $E=E_{1} \otimes E_{2}$ is a graded Clifford module for $V$, with Clifford action $\mathbf{c}\left(v_{1} \oplus v_{2}\right)=\mathbf{c}\left(v_{1}\right) \otimes$ $\mathbf{c}\left(v_{2}\right)$.

Reciprocally, if $V_{1}$ is even dimensional and oriented, any graded Clifford module $E$ on $V=V_{1} \oplus V_{2}$ has the decomposition $E \simeq S_{1} \otimes E_{2}$, where $S_{1}$ is the spinor module for $\mathrm{Cl}\left(V_{1}\right)$ with its canonical grading, and $E_{2}=$ $\operatorname{hom}_{\mathrm{Cl}\left(V_{1}\right)}\left(S_{1}, E\right)$ is a graded $\mathrm{Cl}\left(V_{2}\right)$-module.

\subsection{Euclidean vector bundles and Clifford bundles}

Consider now the case of an Euclidean vector bundle $\mathcal{V} \rightarrow M$ over a manifold $M$. Let $\mathrm{Cl}(\mathcal{V}) \rightarrow M$ be the associated Clifford algebra bundle. A complex $\mathbb{Z} / 2 \mathbb{Z}$-graded vector bundle $\mathcal{E} \rightarrow M$ is a $\mathrm{Cl}(\mathcal{V})$-bundle if there is a bundle algebra odd morphism $c_{\mathcal{E}}: \mathrm{Cl}(\mathcal{V}) \longrightarrow \operatorname{End}(\mathcal{E})$. Thus, for any $x \in M,\left.v \in \mathcal{V}\right|_{x}$, $\mathbf{c}_{\mathcal{E}_{x}}(v):\left.\left.\mathcal{E}\right|_{x} ^{ \pm} \rightarrow \mathcal{E}\right|_{x} ^{\mp}$ is such that $\mathbf{c}_{\mathcal{E}_{x}}(v)^{2}=-\|v\|^{2} \operatorname{Id}_{\left.\mathcal{E}\right|_{x}}$.

Assume that $M$ is a $K$-manifold. Let $\mathcal{V}$ be a $K$-equivariant Euclidean vector bundle on $M$, and $\mathcal{E}$ a $\mathrm{Cl}(\mathcal{V})$-bundle. Then we will always assume that $\mathcal{E}^{ \pm} \rightarrow M$ are $K$-equivariant vector bundles and that $c_{\mathcal{E}}$ is a morphism of $K$-equivariant vector bundles. We will say that $\mathcal{E}$ is a $K$-equivariant Clifford bundle.

If $\mathcal{W}$ is any $K$-equivariant graded complex vector bundle on $M$, and $\mathcal{E}$ a graded Clifford bundle, then $\mathcal{E} \otimes \mathcal{W}$ with Clifford action $\mathbf{c}_{\mathcal{E}} \otimes \operatorname{Id}_{\mathcal{W}}$ will be called the twisted Clifford bundle of $\mathcal{E}$ by $\mathcal{W}$.

Definition 5.6 $A \operatorname{Spin}^{c}$ bundle on $\mathcal{V}$ is a $\mathrm{Cl}(\mathcal{V})$-bundle $\mathcal{S}$ such that, for any $x \in M,\left.\mathcal{S}\right|_{x}$ is an irreducible Clifford module for $\left.\mathcal{V}\right|_{x}$. An orientation on the vector bundle $\mathcal{V}$ induces a (canonical) grading on $\mathcal{S}$. 
If $\mathcal{S}$ is a $\operatorname{Spin}^{c}$-bundle on $\mathcal{V}$ equipped with its canonical grading, we can, as in the linear case, divide any $\mathrm{Cl}(\mathcal{V})$-bundle $\mathcal{F}$ by $\mathcal{S}$ : we write $\mathcal{F}=\mathcal{S} \otimes \mathcal{W}$, and the twisting bundle $\mathcal{W}=\operatorname{hom}_{\mathrm{Cl}(\mathcal{V})}(\mathcal{S}, \mathcal{F})$ inherits a graduation.

Let $K$ be a compact Lie group acting on a manifold $M$. Consider a $K$-equivariant Riemannian metric on $M$ and let $\nu \in \mathrm{T}^{*} M \mapsto \tilde{\nu} \in \mathrm{T} M$ be the corresponding bundle isomorphism.

Definition 5.7 A Clifford bundle on $M$ is a graded Clifford bundle $\mathcal{E}=$ $\mathcal{E}^{+} \oplus \mathcal{E}^{-}$for the Clifford algebra $\mathrm{Cl}(\mathrm{T} M)$.

Let $\mathcal{E}$ be a Clifford bundle on $M$. We define the symbol $\sigma(M, \mathcal{E}) \in$ $\Gamma\left(\mathrm{T}^{*} M, \operatorname{hom}\left(\mathcal{E}^{+}, \mathcal{E}^{-}\right)\right)$by

$$
\sigma(M, \mathcal{E})(x, \nu)=\mathbf{c}_{\mathcal{E}_{x}}(\tilde{\nu}):\left.\left.\mathcal{E}\right|_{x} ^{+} \rightarrow \mathcal{E}\right|_{x} ^{-} .
$$

We always choose $K$-invariant Hermitian structures on $\mathcal{E}^{ \pm}$so that $c_{x}(\tilde{\nu})$ is skew-adjoint. Thus the adjoint of the symbol $\sigma(M, \mathcal{E})$ is the symbol $-\sigma\left(M, \mathcal{E}^{o p}\right)$ (in $\mathcal{E}^{o p}$, we have interchanged the parity on $\mathcal{E}^{+}$and $\mathcal{E}^{-}$). We have

$$
\sigma(M, \mathcal{E})(x, \tilde{\nu}) \sigma\left(M, \mathcal{E}^{o p}\right)(x, \tilde{\nu})=-\|\tilde{\nu}\|_{x}^{2}
$$

so that, if $M$ is compact, $\sigma(M, \mathcal{E})$ is an elliptic symbol.

Remark 5.8 By Bott periodicity theorem, any K-equivariant elliptic symbol on a compact even dimensional oriented manifold $M$ is equal to a symbol $\sigma(M, \mathcal{E})$, for some equivariant Clifford bundle $\mathcal{E}$, in the equivariant $K$-theory of TM. We will prove a localization formula for the symbols $\sigma(M, \mathcal{E})$. They could be rephrased for any elliptic symbol, but this would require more notational framework.

\subsection{Choice of metrics}

Let us consider the role of the metric in our setting. Let $(M, \mathcal{V}, \mathcal{E})$ be a $K$-equivariant Clifford data $: \mathcal{V} \rightarrow M$ is an Euclidean vector bundle and $\mathcal{E} \rightarrow M$ is a graded $\mathrm{Cl}(\mathcal{V})$-bundle. The metric $g$ on the Euclidean vector bundle $\mathcal{V} \rightarrow M$ determines a bundle map $g_{\sharp}: \mathcal{V} \rightarrow \mathcal{V}^{*}$. If we take another metric $g^{\prime}$ on $\mathcal{V} \rightarrow M$ the bundle map $A:=\left(g_{\sharp}\right)^{-1} \circ\left(g^{\prime}\right)_{\sharp}$ is symmetric positive definite for $g$. Hence, $C=A^{1 / 2}$ is a well-defined bundle map, symmetric with respect to $g$. It defines an isometry $C:(\mathcal{V}, g) \rightarrow\left(\mathcal{V}, g^{\prime}\right)$, and then a canonical isomorphism $\mathrm{Cl}(\mathcal{V}, g) \simeq \mathrm{Cl}\left(\mathcal{V}, g^{\prime}\right)$. So we will often speak of a Clifford bundle $\mathcal{E}$ on $\mathcal{V}$ without specifying the metric on $\mathcal{V}$.

In particular if $\pi: \mathcal{H} \rightarrow B$ is a Hermitian vector bundle on a Riemannian $K$-manifold $B$, using a $K$-invariant connection, we can choose a metric on 
$\mathrm{TH}$ such that $\mathrm{TH}$ is isomorphic to the orthogonal direct sum $\pi^{*} \mathrm{~T} B \oplus \pi^{*} \mathcal{H}$ of Euclidean vector bundles. Then any Clifford module for $\mathrm{TH}$ is of the form

$$
\left.\pi^{*} \mathcal{E}\right|_{B} \otimes \pi^{*} \bigwedge \overline{\mathcal{H}}
$$

where $\left.\mathcal{E}\right|_{B}$ is a Clifford module for T $B$, and, for $\pi(n)=x$, the Clifford map $\mathbf{c}_{n}$ is equal to $\mathbf{c}_{x}^{1} \otimes \mathbf{c}_{x}^{2}$, where

$$
\begin{aligned}
& \mathbf{c}_{x}^{1}: \mathrm{T}_{x} B \longrightarrow \operatorname{End}\left(\left.\left.\mathcal{E}\right|_{B}\right|_{x}\right), \\
& \mathbf{c}_{x}^{2}:\left.\mathcal{H}\right|_{x} \longrightarrow \operatorname{End}\left(\left.\bigwedge \overline{\mathcal{H}}\right|_{x}\right) .
\end{aligned}
$$

\subsection{The Bott symbol and direct image}

Let $N$ be an Euclidean vector space. The dual space $N^{*}$ inherits an Euclidean structure through the identification $N^{*} \rightarrow N, \eta \rightarrow \tilde{\eta}$ given by the relation : $\langle\eta, n\rangle=(\tilde{\eta}, n)$. The vector space $N \oplus N^{*}$ is an Euclidean space of even dimension that we identify to $N_{\mathbb{C}}$ by $n \oplus \eta \rightarrow n \oplus i \tilde{\eta}$. The group $O(N)$ acting diagonally on $N \oplus N^{*}$ becomes a subgroup of $U\left(N_{\mathbb{C}}\right)$, and acts on $S_{N}:=\bigwedge N_{\mathbb{C}}$ via the natural representation of $U\left(N_{\mathbb{C}}\right)$ in $\bigwedge N_{\mathbb{C}}$.

Recall the definition of the Bott symbol. We denote by $\mathbf{c}_{N_{\mathbb{C}}}$ the Clifford representation of $N_{\mathbb{C}}$ in $S_{N}$. Then $\mathbf{c}_{N_{\mathbb{C}}}\left(n \oplus i n^{\prime}\right)$ satisfies $\mathbf{c}_{N_{\mathbb{C}}}\left(n \oplus i n^{\prime}\right)^{2}=$ $-\left(\|n\|^{2}+\left\|n^{\prime}\right\|^{2}\right) \mathrm{Id}$.

Consider the trivial bundle $\mathcal{S}=N \times S_{N}$. We define the symbol $\operatorname{Bott}\left(N_{\mathbb{C}}\right) \in$ $\Gamma\left(\mathrm{T}^{*} N, \operatorname{hom}\left(\mathcal{S}^{+}, \mathcal{S}^{-}\right)\right)$by

$$
\operatorname{Bott}\left(N_{\mathbb{C}}\right)(n, \eta)=\mathbf{c}_{N_{\mathbb{C}}}(n \oplus i \tilde{\eta}): S_{N}^{+} \rightarrow S_{N}^{-} .
$$

Here $n \in N, \eta \in N^{*}$. The characteristic support of $\operatorname{Bott}\left(N_{\mathbb{C}}\right)$ is $\{0\}$, so $\operatorname{Bott}\left(N_{\mathbb{C}}\right)$ is an elliptic symbol on $N$ called the Bott symbol. It is equivariant for the action of $O(N)$ and $\operatorname{Index} N_{O(N)}^{N}\left(\operatorname{Bott}\left(N_{\mathbb{C}}\right)\right)$ is the trivial representation of $O(N)$ [4].

Let $\mathcal{N} \rightarrow \mathcal{X}$ be an Euclidean vector bundle. The orthogonal frame bundle $P$ of $\mathcal{N}$ is a $\mathrm{O}(n)$-principal bundle such that $\mathcal{N}=P \times_{\mathrm{O}(n)} N$, with $N=\mathbb{R}^{n}$. Using the notion of fiber product introduced in Section 4.4 with the group $S=\{1\}$, we define the pushforward morphism $i_{!}: \mathbf{K}_{K}^{0}\left(\mathrm{~T}_{K}^{*} \mathcal{X}\right) \longrightarrow$ $\mathbf{K}_{K}^{0}\left(\mathrm{~T}_{K}^{*} \mathcal{N}\right)$ by the relation

$$
i_{!}(\sigma):=\sigma \diamond \operatorname{Bott}\left(N_{\mathbb{C}}\right),
$$

if $\sigma$ is a transversally elliptic symbol on $\mathcal{X}$.

The following theorem is a consequence of Lemma 4.6. 


\section{Theorem 5.9 (Invariance of the index by push-forward)}

$$
\operatorname{Index}_{K}^{\mathcal{X}}(\sigma)=\operatorname{Index}_{K}^{\mathcal{N}}\left(i_{!}(\sigma)\right) .
$$

A symbol representing $i_{!}(\sigma)$ is as follows. We write an element $n \in \mathcal{N}$ as $(x, v)$ with $x \in \mathcal{X}$ and $\left.v \in \mathcal{N}\right|_{x}$. We choose a $K$-invariant connection on the bundle $\mathcal{N} \rightarrow \mathcal{X}$, which allows us to parameterize $\mathrm{T}^{*} \mathcal{N}=\{(n,(\xi, \eta))\}$, with $\xi \in \mathrm{T}_{x}^{*} \mathcal{X},\left.\eta \in \mathcal{N}^{*}\right|_{x}$ : here $\xi$ is lifted to an horizontal element of $\mathrm{T}_{n}^{*} \mathcal{N}$ using the connection, so $(\xi, \eta)$ parameterize any element in $\mathrm{T}_{n}^{*} \mathcal{N}$. Let $\sigma \in \Gamma\left(\mathrm{T}^{*} \mathcal{X}, \operatorname{hom}\left(\mathcal{E}^{+}, \mathcal{E}^{-}\right)\right)$be a symbol on $\mathcal{X}$. Then the symbol

$$
((x, v),(\xi, \eta)) \mapsto \sigma(x, \xi) \otimes \mathbf{c}_{x}(v \oplus i \tilde{\eta})
$$

represents $i_{!}(\sigma)$. Here $\mathbf{c}_{x}$ is the Clifford representation of $\left.\mathcal{N}\right|_{x} \otimes \mathbb{C}$ on $\bigwedge\left(\left.\mathcal{N}\right|_{x} \otimes\right.$ $\mathbb{C})$, so $\left(\mathbf{c}_{x}(v \oplus i \tilde{\eta})\right)^{2}=-\left(\|v\|^{2}+\|\eta\|^{2}\right) \operatorname{Id}$ and $\operatorname{Char}(i !(\sigma))=\operatorname{Char}(\sigma)$.

\subsection{Dirac operators and equivariant indices}

Let $\mathcal{E} \rightarrow M$ be a $K$-equivariant graded Clifford bundle on a compact Riemannian $K$-manifold $M$ of even dimension.

Definition 5.10 We denote by $\mathcal{Q}_{K}(M, \mathcal{E}) \in R(K)$ the index of the symbol $\sigma(M, \mathcal{E})$.

If $K$ is the trivial group, we denote $\mathcal{Q}_{K}(M, \mathcal{E})$ simply by $\mathcal{Q}(M, \mathcal{E})$. This is an integer.

We can realize $\mathcal{Q}_{K}(M, \mathcal{E})$ as the index of a Dirac operator. We choose $K$ invariant connections on $\mathcal{E}^{ \pm}$such that the Dirac operator $\mathcal{D}_{\mathcal{E}}^{ \pm}: \Gamma\left(M, \mathcal{E}^{ \pm}\right) \rightarrow$ $\Gamma\left(M, \mathcal{E}^{\mp}\right)[8]$ verifies $\left(\mathcal{D}_{\mathcal{E}}^{+}\right)^{*}=\mathcal{D}_{\mathcal{E}}^{-}$. The principal symbol of $\mathcal{D}_{\mathcal{E}}^{+}$is $\sigma(M, \mathcal{E})$. Thus $\mathcal{Q}_{K}(M, \mathcal{E})$ can be realized as the difference of the two finite dimensional representation spaces $\left[\operatorname{ker} \mathcal{D}_{\mathcal{E}}^{+}\right]-\left[\operatorname{ker} \mathcal{D}_{\mathcal{E}}^{-}\right]$and we also say that $\mathcal{Q}_{K}(M, \mathcal{E})$ is the equivariant index of the Dirac operator $\mathcal{D}_{\mathcal{E}}^{+}$.

For example, if $M$ is any $K$-manifold, and $\mathcal{E}=\bigwedge\left(T M \otimes_{\mathbb{R}} \mathbb{C}\right)$, a Dirac operator with principal symbol $\sigma(M, \mathcal{E})$ can be taken as $d+d^{*}$ where $d$ is the de Rham differential. If $K$ is connected, $\mathcal{Q}_{K}(M, \mathcal{E})$ is a multiple of the trivial representation of $K$, the multiplicity being the Euler characteristic of $M$.

Example 5.11 An important example is when $M$ is a complex manifold with complex structure $J \in \Gamma(M, \mathrm{~T} M)$. Then $\mathrm{T} M \otimes_{\mathbb{R}} \mathbb{C}$ breaks into two pieces, the holomorphic and anti-holomorphic tangent spaces

$$
\mathrm{T} M \otimes_{\mathbb{R}} \mathbb{C}=\mathrm{T}^{1,0} M \oplus \mathrm{T}^{0,1} M
$$


on which $J$ acts by $i$, and $-i$, respectively. We consider a Riemannian metric on $\mathrm{T} M$ such that $g(J v, J v)=g(v, v)$. Let $\mathcal{W} \rightarrow M$ be a holomorphic vector bundle on $M$. Then the vector bundle of $\mathcal{W}$-valued anti-holomorphic differential forms

$$
\mathcal{E}=\bigwedge\left(\mathrm{T}^{0,1} M\right)^{*} \otimes \mathcal{W}
$$

is a graded Clifford bundle. This bundle is isomorphic to $\bigwedge_{J} \mathrm{~T} M \otimes \mathcal{W}$, with Clifford action described by the formula (5.9). A Dirac operator with principal symbol $\sigma(M, \mathcal{E})$ is $\bar{\partial}_{\mathcal{W}}+\left(\bar{\partial}_{\mathcal{W}}\right)^{*}$ where $\bar{\partial}_{\mathcal{W}}$ is the Dolbeaut operator. Assume that $K$ acts by holomorphic transformations on $M, \mathcal{W}$. Then $\mathcal{Q}_{K}(M, \mathcal{E})=\sum_{j}(-1)^{j} H^{j}(M, \mathcal{O}(\mathcal{W}))$ is the alternate sum of the cohomology groups of the sheaf of holomorphic sections of $\mathcal{W}$.

Example 5.12 Let $K$ be a compact connected Lie group, and $T \subset K$ be a maximal torus. We consider the flag manifold $\mathbb{F}:=K / T$ with base point $\bar{e} \in K / T$. The choice of a Weyl chamber $\mathfrak{t}_{>_{0}}^{*} \subset \mathfrak{t}^{*}$ determines a $T$-invariant complex structure $J$ on $\mathrm{T}_{\bar{e}} \mathbb{F} \simeq \mathfrak{k} / \mathfrak{t}$ such that the complex $T$-module $\mathrm{T}^{1,0} \mathbb{F}$ is equal to $\oplus_{\alpha>0} \mathbb{C}_{\alpha}$, where $\{\alpha>0\}$ denotes the set of positive roots. The complex structure $J$ defines an integrable complex structure on the flag manifold $\mathbb{F}$ (still denoted $J$ ). Let $\Lambda \subset \mathfrak{t}^{*}$ be the weight lattice. Any weight $\mu \in \Lambda$ determines an holomorphic line bundle $\left[\mathbb{C}_{\mu}\right]:=K \times_{T} \mathbb{C}_{\mu}$ on $\mathbb{F}$. If $\mu$ is dominant, the Borel-Weil Theorem tells us that $\mathcal{Q}_{K}\left(\mathbb{F}, \bigwedge_{J} \mathrm{TF} \otimes\left[\mathbb{C}_{\mu}\right]\right)$ is equal to the irreducible representation of $K$ with highest weight $\mu$.

\subsection{Horizontal Clifford bundle}

Let $G$ and $K$ be two compact Lie groups. Let $P$ be a manifold provided with an action of $G \times K$. Assume that the action of $K$ is free and let $M=P / K$. We are in the situation described in Subsection 4.2, and we use the same notations.

For the tangent bundle, the choice of a $G$-invariant connection on the principal bundle $P \rightarrow M$ defines an invariant decomposition T $P=\pi^{*} \mathrm{~T} M \oplus$ $P \times \mathfrak{k}$; if a metric is chosen on $M$, we define the metric on $P$ by declaring that this decomposition is orthogonal.

Let $\mathcal{E}$ be a $G$-equivariant graded Clifford bundle on $M$. We obtain from $\mathcal{E}$ a symbol

$$
\sigma_{\text {hor }}(P, \mathcal{E}) \in \Gamma\left(\mathrm{T}^{*} P, \operatorname{hom}\left(\pi^{*} \mathcal{E}^{+}, \pi^{*} \mathcal{E}^{-}\right)\right)
$$

by defining $\sigma_{\text {hor }}(P, \mathcal{E})(p, \eta)=\sigma(M, \mathcal{E})(x, \tilde{\theta})$ where $(x, \theta):=\bar{\pi}(p, \eta)$.

Assume $P$ compact. It is clear that $\sigma_{h o r}(P, \mathcal{E})$ is a $K$-transversally elliptic symbol on $P$. It will be called the horizontal Dirac symbol. Furthermore, 
this symbol is $G$-equivariant, and for any irreducible representation $V_{\mu}$ of $K$, the finite dimensional space

$$
\left[\operatorname{Index}_{G \times K}^{P}\left(\sigma_{h o r}(P, \mathcal{E})\right) \otimes V_{\mu}^{*}\right]^{K}
$$

carries a representation of $G$.

We form the $G$-equivariant complex vector bundle $\mathcal{V}_{\mu}:=P \times_{K} V_{\mu}$ on $M$, and the twisted Clifford bundle $\mathcal{E} \otimes \mathcal{V}_{\mu}$. We consider the elliptic symbol $\sigma\left(M, \mathcal{E} \otimes \mathcal{V}_{\mu}\right)$ on $M$.

The following result follows easily from the Peter-Weyl decomposition of $\Gamma\left(P, \pi^{*} \mathcal{E}\right)$ under the free action of $K$. It is a particular case of the free action property of transversally elliptic symbols.

Theorem 5.13 In $\hat{R}(G \times K)$ we have

$$
\operatorname{Index}_{G \times K}^{P}\left(\sigma_{h o r}(P, \mathcal{E})\right)=\sum_{\mu \in \hat{K}} \mathcal{Q}_{G}\left(M, \mathcal{E} \otimes \mathcal{V}_{\mu}^{*}\right) \otimes V_{\mu}
$$

In particular $\left[\operatorname{Index}_{G \times K}^{P}\left(\sigma_{\text {hor }}(P, \mathcal{E})\right)\right]^{K}=\mathcal{Q}_{G}(M, \mathcal{E})$.

Consider now the case of a $G \times K$ Riemannian manifold $P$, but assume now that $K$ acts only infinitesimally freely: for any $p \in P$, the stabilizer $K_{p}$ is a finite subgroup. So $M=P / K$ is an orbifold, but maybe not a manifold. Consider $\mathcal{H}$, the subbundle of $\mathrm{T} P$ defined by the orthogonal decomposition $\mathrm{T} P=\mathcal{H} \oplus P \times \mathfrak{k}$ and call $\mathcal{H}$ the horizontal tangent bundle.

Definition 5.14 • If $\mathcal{E}$ is a graded Clifford bundle for the horizontal tangent bundle $\mathcal{H}$, equivariant for the $G \times K$-action, we define $\sigma_{\text {hor }}(P, \mathcal{E})(p, \nu)=$ $\mathbf{c}_{\mathcal{E}_{p}}\left(\tilde{\mathcal{\nu}}_{\mathcal{H}}\right)$ where $\tilde{\nu}_{\mathcal{H}}$ is the horizontal component of $\tilde{\nu} \in \mathrm{T}_{p} P$.

- We define $\mathcal{Q}_{G}(M, \mathcal{E})$ by the formula

$$
\mathcal{Q}_{G}(M, \mathcal{E}):=\left[\operatorname{Index}_{G \times K}^{P}\left(\sigma_{h o r}(P, \mathcal{E})\right)\right]^{K} .
$$

It can be shown, see [48], that this is indeed the equivariant index of a Dirac operator on the $G$-orbifold $M$ in the sense of Kawasaki [19].

\section{Deformation à la Witten of Dirac operators}

We start to discuss the real purpose of this paper. We will study the deformation à la Witten of elliptic symbols $\sigma(M, \mathcal{E})$ by vector fields associated to $K$-equivariant maps $\Phi: M \rightarrow \mathfrak{k}^{*}$.

We recall that we chose an invariant scalar product in $\mathfrak{k}$ providing an identification $\mathfrak{k} \simeq \mathfrak{k}^{*}$. 


\subsection{Kirwan vector field}

Let $M$ be a $K$-manifold and let $\Phi: M \rightarrow \mathfrak{k}^{*}$ be an equivariant map.

Definition 6.1 • The Kirwan vector field associated to $\Phi$ is defined by

$$
\kappa_{\Phi}(m)=-\Phi(m) \cdot m, \quad m \in M .
$$

- We denote by $Z_{\Phi}$ the set of zeroes of $\kappa_{\Phi}$. Thus $Z_{\Phi}$ is a $K$-invariant closed subset of $M$.

The $Z_{\Phi}$ is not necessarily smooth. If $0 \in \Phi(M)$, then clearly $Z_{\Phi}$ contains $\Phi^{-1}(0)$.

In order to describe more precisely $Z_{\Phi}$, we consider the set $\left\{\left(\mathfrak{k}_{i}\right), i \in I\right\}$ of conjugacy classes of subalgebras of $\mathfrak{k}$ representing the infinitesimal stabilizers of the $K$-action on $M$ : if $M$ is compact, the set $I$ is finite. We denoted by $M_{\mathfrak{k}_{i}}$ the submanifold of points $m \in M$ such that $\mathfrak{k}_{i}=\mathfrak{k}_{m}$. We see that $Z_{\Phi} \cap M_{\mathfrak{k}_{i}}=M_{\mathfrak{k}_{i}} \cap \Phi^{-1}\left(\mathfrak{k}_{i}^{*}\right)$, hence

$$
Z_{\Phi}=\bigsqcup_{i \in I} K\left(M_{\mathfrak{k}_{i}} \cap \Phi^{-1}\left(\mathfrak{k}_{i}^{*}\right)\right)
$$

where $\mathfrak{k}_{i}^{*}$ is viewed as a subspace of $\mathfrak{k}^{*}$ thanks to the scalar product.

Definition 6.2 An equivariant map $\Phi: M \rightarrow \mathfrak{k}^{*}$ is called a moment map if there exists an invariant (real) 2-form $\Omega$ on $M$ satisfying the relations

$$
\iota\left(X_{M}\right) \Omega+d\langle\Phi, X\rangle=0 \quad \text { and } \quad d \Omega=0
$$

for $X \in \mathfrak{k}$. The data $(\Omega, \Phi)$ on $M$ is called a weak Hamiltonian structure.

Classically, a moment map in the sense of Hamiltonian geometry requires that $\Omega$ is non degenerate. In this text, we will see that some localization results can be proven without this strong hypothesis.

A particularly important example of moment map is provided by the following construction.

Definition 6.3 Let $L$ be a $K$-equivariant Hermitian line bundle with Hermitian connection $\nabla$ with curvature $R_{L}$. Let $\Omega_{L}=\frac{1}{i} R_{L}$. Then $\Omega_{L}$ is a closed real two form. This data determines a moment map

$$
\Phi_{L}: M \rightarrow \mathfrak{k}^{*}
$$

by the relation $\mathcal{L}(X)-\nabla_{X_{M}}=i\left\langle\Phi_{L}, X\right\rangle$, for all $X \in \mathfrak{k}$. Here $\mathcal{L}(X)$ is the infinitesimal action of $X \in \mathfrak{k}$ on the sections of $L$. The relation $\iota\left(X_{M}\right) \Omega_{L}+$ $d\left\langle\Phi_{L}, X\right\rangle=0$ follows. 
Remark 6.4 The subset $\Phi_{L}^{-1}(0)$ of the zeroes of the associated Kirwan vector field has some particular importance when $L$ is an ample bundle on a projective manifold provided with an action of $K$. In this case, if $\Phi_{L}$ is the moment map associated to the projective embedding, $\Phi_{L}^{-1}(0) / K$ is isomorphic to the geometric invariant theory (GIT) quotient $M / / K_{\mathbb{C}}$ of $M$ by $K_{\mathbb{C}}$, and is provided with structure of projective variety with corresponding line bundle $L / / K_{\mathbb{C}}$. When $M / / K_{\mathbb{C}}$ is smooth, Guillemin and Sternberg [16] have proved the first $[Q, R]=0$ identity :

$$
\left[H^{0}(M, L)\right]^{K} \simeq H^{0}\left(M / / K_{\mathbb{C}} ; L / / K_{\mathbb{C}}\right) .
$$

Remark 6.5 If $\Phi$ is a moment map, by Equation (6.14), we have

$$
d\|\Phi\|^{2}=2 \iota\left(\kappa_{\Phi}\right) \Omega .
$$

Thus the set $Z_{\Phi}$ is contained in the critical set of the function $\|\Phi\|^{2}$ : if $\beta \in \Phi\left(Z_{\Phi}\right)$, then $\|\beta\|^{2}$ is a critical value of the square of the moment map $\Phi$.

One of the properties that a moment map shares with the moment map of a Hamiltonian action is the following.

Proposition 6.6 • For any $X \in \mathfrak{k}$, the function $\langle\Phi, X\rangle$ is constant on each connected component of the manifold $M^{X}$.

- If 0 is a regular value of a moment map $\Phi$, then the action of $K$ is infinitesimally free on the submanifold $\Phi^{-1}(0)$. The reduced space $M_{\text {red }}=$ $\Phi^{-1}(0) / K$ is an orbifold.

- Conversely, if $\Phi$ is the moment map of a Hamiltonian action, and the action of $K$ is infinitesimally free on the submanifold $\Phi^{-1}(0)$, then 0 is a regular value of $\Phi$.

Proof. Equation (6.14) tells us that for a point $m \in M$ where $X_{M}(m)=0$, the differential $\mathrm{T}_{m} \Phi$ sends $\mathrm{T}_{m} M$ to $(\mathbb{R} X)^{\perp} \subset \mathfrak{k}^{*}$. Our three points follow from this simple fact.

We note that any invariant 1-form $\alpha$ defines a moment map $\varphi_{\alpha}: M \rightarrow \mathfrak{k}^{*}$ by the relation : $\left\langle\varphi_{\alpha}, X\right\rangle=\iota\left(X_{M}\right) \alpha, X \in \mathfrak{k}$ : the corresponding 2-form is $d \alpha$.

We will use the following equivalence relation between moment maps on $M$ :

$$
\Phi \sim \Phi^{\prime} \Longleftrightarrow \exists \text { an invariant } 1 \text {-form } \alpha \text { such that } \Phi-\Phi^{\prime}=\varphi_{\alpha} .
$$


Let us describe $\Phi\left(Z_{\Phi}\right)$ when $\Phi$ is a moment map. As before, let $\left\{\mathfrak{k}_{i}, i \in I\right\}$ be a set of representatives of the infinitesimal stabilizers of the $K$-action on $M$, and let $M_{\mathfrak{k}_{i}}$ be the submanifold of points $m \in M$ such that $\mathfrak{k}_{i}=\mathfrak{k}_{m}$. If $\mathcal{X}$ is a connected component of $M_{\mathfrak{k}_{i}}$, Relations (6.14) imply that the image $\Phi(\mathcal{X})$ is contained in an affine space $A(\mathcal{X}, \Phi) \subset \mathfrak{k}^{*}$ with direction $\mathfrak{k}_{i}^{\perp}$. The orthogonal projection $\beta(\mathcal{X}, \Phi)$ of 0 on the affine space of $A(\mathcal{X}, \Phi)$ belongs to $\mathfrak{k}_{i}^{*}$, viewed as a subspace of $\mathfrak{k}^{*}$ thanks to the scalar product.

Lemma 6.7 The set $\Phi\left(Z_{\Phi}\right)$ is contained in

$$
C_{\Phi}:=\bigcup_{i \in I} \bigcup_{\mathcal{X} \subset M_{\mathfrak{k}_{i}}} K \cdot \beta(\mathcal{X}, \Phi) .
$$

More precisely, the orbit $K \cdot \beta(\mathcal{X}, \Phi)$ is contained in $\Phi\left(Z_{\Phi}\right)$ if and only if $\beta(\mathcal{X}, \Phi)$ belongs to $\Phi(\mathcal{X})$.

Proof. The proof is left to the reader.

Concerning the set $C_{\Phi}$, we have the useful remark.

Proposition 6.8 Let $\Phi, \Phi^{\prime}$ be two moment maps on the $K$-manifold $M$.

- If $\Phi \sim \Phi^{\prime}$ then $C_{\Phi}=C_{\Phi^{\prime}}$.

- If $M$ is compact, $C_{\Phi}$ is a finite union of coadjoint orbits.

- If $Z_{\Phi}$ is compact, then $\Phi\left(Z_{\Phi}\right)$ is a finite union of coadjoint orbits.

Proof. The first point is due to the fact that $A(\mathcal{X}, \Phi)=A\left(\mathcal{X}, \Phi+\varphi_{\alpha}\right)$ for any invariant 1-form $\alpha$ and any connected component $\mathcal{X}$ of $M_{\mathfrak{k}_{i}}$. If $M$ is compact, the set $I$ is finite and each submanifold $M_{\mathfrak{k}_{i}}$ has a finite number of connected components, so there is a finite number of terms $\beta(\mathcal{X}, \Phi)$. If $Z_{\Phi}$ is compact, we consider an invariant neighborhood $\mathcal{U}$ of $Z_{\Phi}$ such that $\overline{\mathcal{U}}$ is compact. The subset $I_{\mathcal{U}} \subset I$ formed by the element $i \in I$ such that $M_{\mathfrak{k}_{i}} \cap \mathcal{U} \neq \emptyset$ is finite, and we see that $\Phi\left(Z_{\Phi}\right)$ is contained in

$$
C_{\Phi}^{\mathcal{U}}:=\bigcup_{i \in I_{\mathcal{U}}} \bigcup_{\mathcal{X} \subset M_{\mathfrak{k}_{i}} \cap \mathcal{U}} K \cdot \beta(\mathcal{X}, \Phi)
$$

Since $C_{\Phi}^{\mathcal{U}}$ is a finite union of coadjoint orbits, the last assertion is proved.

For any moment map $\Phi$, if $Z_{\Phi}$ is compact, it admits a finite decomposition $Z_{\Phi}=\coprod_{\mathcal{O}} Z_{\mathcal{O}}$ where $Z_{\mathcal{O}}=Z_{\Phi} \cap \Phi^{-1}(\mathcal{O})$. 
Definition 6.9 We denote by $\mathcal{B}(\Phi)$ the set of coadjoint orbits contained in $\Phi\left(Z_{\Phi}\right)$.

In practice, we parameterize the set $\mathcal{B}(\Phi)$ by a finite set $\mathcal{B}$ contained in $\mathfrak{k}^{*}: \Phi\left(Z_{\Phi}\right)=\coprod_{\beta \in \mathcal{B}} K \beta$ and then

$$
Z_{\Phi}=\coprod_{\beta \in \mathcal{B}} Z_{\beta}
$$

where $Z_{\beta}=K\left(M^{\beta} \cap \Phi^{-1}(\beta)\right)$.

\subsection{Deforming a symbol}

Let $M$ be a $K$-manifold. We do not necessarily assume $M$ to be compact, and the compact Lie group $K$ nor $M$ are necessarily connected. Let $\Phi$ : $M \rightarrow \mathfrak{k}^{*}$ be an equivariant map, and let $\mathcal{E}$ be a graded Clifford bundle on $M$.

Definition 6.10 The symbol $\sigma(M, \mathcal{E}, \Phi)$ pushed by the vector field $\kappa_{\Phi}$ is the symbol on $M$ defined by

$$
\sigma(M, \mathcal{E}, \Phi)(m, \nu)=\sigma(M, \mathcal{E})\left(m, \tilde{\nu}-\kappa_{\Phi}(m)\right)=\mathbf{c}_{\mathcal{E}_{m}}\left(\tilde{\nu}-\kappa_{\Phi}(m)\right)
$$

for any $(m, \nu) \in \mathrm{T}^{*} M$.

Note that $\sigma(M, \mathcal{E}, \Phi)(m, \nu)$ is invertible except if $\tilde{\nu}=\kappa_{\Phi}(m)$. If furthermore $(m, \nu)$ belongs to the subset $\mathrm{T}_{K}^{*} M$ of cotangent vectors orthogonal to the $K$-orbits, then $\nu=0$ and $\kappa_{\Phi}(m)=0$. Indeed, as $\kappa_{\Phi}(m)$ is tangent to $K \cdot m$, the two equations $\tilde{\nu}=\kappa_{\Phi}(m)$ and $\left\langle\nu, \kappa_{\Phi}(m)\right\rangle=0$ imply $\tilde{\nu}=\kappa_{\Phi}(m)=0$. So we note that $(m, \nu) \in \operatorname{Char}(\sigma(M, \mathcal{E}, \Phi)) \cap \mathrm{T}_{K}^{*} M$ if and only if $\nu=0$ and $\kappa_{\Phi}(m)=0$. Hence $\sigma(M, \mathcal{E}, \Phi)$ is transversally elliptic whenever $Z_{\Phi}$ is compact.

Definition 6.11 If $Z_{\Phi}$ is compact, we define $\mathcal{Q}_{K}(M, \mathcal{E}, \Phi) \in \hat{R}(K)$ as the equivariant index of the transversally elliptic symbol $\sigma(M, \mathcal{E}, \Phi) \in \mathbf{K}_{K}^{0}\left(\mathrm{~T}_{K}^{*} M\right)$.

When $M$ is compact, it is clear that the classes of the symbols $\sigma(M, \mathcal{E}, \Phi)$ and $\sigma(M, \mathcal{E})$ are equal in $\mathbf{K}_{K}^{0}\left(\mathrm{~T}_{K}^{*} M\right)$, hence the equivariant indices $\mathcal{Q}_{K}(M, \mathcal{E})$ and $\mathcal{Q}_{K}(M, \mathcal{E}, \Phi)$ are equal.

For any $K$-invariant, relatively compact, open subset $\mathcal{U} \subset M$ such that $\mathcal{U} \cap Z_{\Phi}$ is closed ${ }^{4}$ in $M$, we see that the restriction $\sigma(M, \mathcal{E}, \Phi) \mid \mathcal{U}$ is a transversally elliptic symbol on $\mathcal{U}$, and so its equivariant index is a well defined element in $\hat{R}(K)$, independent of the choice of such a $\mathcal{U}$.

${ }^{4} \mathcal{U} \cap Z_{\Phi}$ is then compact. 
Definition 6.12 $-A$ closed $K$ invariant subset $Z \subset Z_{\Phi}$ is called a component if it is a union of connected components of $Z_{\Phi}$.

- For a compact component $Z$ of $Z_{\Phi}$, we denote by

$$
\sigma(M, \mathcal{E}, Z, \Phi) \in \mathbf{K}_{K}^{0}\left(\mathrm{~T}_{K}^{*} M\right)
$$

the image of $\left.\sigma(M, \mathcal{E}, \Phi)\right|_{\mathcal{U}} \in \mathbf{K}_{K}^{0}\left(\mathrm{~T}_{K}^{*} \mathcal{U}\right)$ by the pushforward morphism $\mathbf{K}_{K}^{0}\left(\mathrm{~T}_{K}^{*} \mathcal{U}\right) \rightarrow \mathbf{K}_{K}^{0}\left(\mathrm{~T}_{K}^{*} M\right)$ attached to a relatively compact invariant neighborhood $\mathcal{U}$ of $Z$ satisfying $\mathcal{U} \cap Z_{\Phi}=Z$.

- For a compact component $Z$ of $Z_{\Phi}$, we denote by

$$
\mathcal{Q}_{K}(M, \mathcal{E}, Z, \Phi) \in \hat{R}(K)
$$

the equivariant index of $\sigma(M, \mathcal{E}, Z, \Phi)$. By definition, if $Z=\emptyset$, then $\mathcal{Q}_{K}(M, \mathcal{E}, \emptyset, \Phi)=0$.

In particular, if $\Phi: M \rightarrow \mathfrak{k}^{*}$ is a proper moment map, we can define

$$
\mathcal{Q}_{K}\left(M, \mathcal{E}, \Phi^{-1}(0), \Phi\right) \in \hat{R}(K) .
$$

By taking its invariant part $\left[\mathcal{Q}_{K}\left(M, \mathcal{E}, \Phi^{-1}(0), \Phi\right)\right]^{K} \in \mathbb{Z}$, we will define in a natural way invariants on the reduced "manifold" $M_{\text {red }}=\Phi^{-1}(0) / K$.

The simplest example of a pushed symbol $\sigma(M, \mathcal{E}, \Phi)$ is when the map $\Phi$ is constant, equal to a $K$-invariant element $\beta \in \mathfrak{k}^{*} \simeq \mathfrak{k}$. The vector field $m \mapsto \beta \cdot m$ is then $K$-equivariant, and we define

$$
\sigma(M, \mathcal{E}, \beta)(m, \nu)=\sigma(M, \mathcal{E})(m, \tilde{\nu}+\beta \cdot m) .
$$

The first example of pushed symbol $\sigma(M, \mathcal{E}, \beta)$ is the Atiyah symbol that we will study in the next section.

We end this section by considering the case where $\Phi$ is a moment map such that $Z_{\Phi}$ is compact. For any $\mathcal{O} \subset C_{\Phi}$, the set $Z_{\mathcal{O}}=Z_{\Phi} \cap \Phi^{-1}(\mathcal{O})$ is a compact component of $Z_{\Phi}$, hence we may consider the localized index $\mathcal{Q}_{K}\left(M, \mathcal{E}, Z_{\mathcal{O}}, \Phi\right)$. This generalized character is non-zero only if $Z_{\mathcal{O}} \neq \emptyset$, and this happens only for a finite number of orbits (those included in $\Phi\left(Z_{\Phi}\right)$ ).

Thanks to the excision property, we get the first form of the localization theorem :

$$
\mathcal{Q}_{K}(M, \mathcal{E}, \Phi)=\sum_{\mathcal{O} \subset C_{\Phi}} \mathcal{Q}_{K}\left(M, \mathcal{E}, Z_{\mathcal{O}}, \Phi\right)
$$

The aim of Section 7.2 is the description by induction of the representations $\mathcal{Q}_{K}\left(M, \mathcal{E}, Z_{\mathcal{O}}, \Phi\right)$.

Let us show that, when $M$ is compact, the character $\mathcal{Q}_{K}\left(M, \mathcal{E}, Z_{\mathcal{O}}, \Phi\right)$ depends only of the class of $\Phi$ relative to the equivalence relation (6.15). 
Proposition 6.13 Let $\Phi \sim \Phi^{\prime}$ be two moment maps on a compact $K$ manifold. Let $Z_{\mathcal{O}} \subset Z_{\Phi}$ and $Z_{\mathcal{O}}^{\prime}=Z_{\Phi^{\prime}} \cap\left(\Phi^{\prime}\right)^{-1}(\mathcal{O})$ be the components attached to $\mathcal{O} \in C_{\Phi}=C_{\Phi^{\prime}}$. Then we have

$$
\mathcal{Q}_{K}\left(M, \mathcal{E}, Z_{\mathcal{O}}, \Phi\right)=\mathcal{Q}_{K}\left(M, \mathcal{E}, Z_{\mathcal{O}}^{\prime}, \Phi^{\prime}\right)
$$

Proof. Let $\alpha$ be the invariant 1-form such that $\Phi^{\prime}-\Phi=\varphi_{\alpha}$. We consider the family of moment maps $\Phi^{t}=\Phi+t \varphi_{\alpha}, t \in \mathbb{R}$. We denote simply by $\sigma^{t}$ the symbol $\sigma\left(M, \mathcal{E}, \Phi^{t}\right)$. For any $t$, consider the component $Z_{\mathcal{O}}^{t}=Z_{\Phi^{t}} \cap\left(\Phi^{t}\right)^{-1}(\mathcal{O})$ and the generalized character

$$
\mathcal{Q}^{t}:=\mathcal{Q}_{K}\left(M, \mathcal{E}, Z_{\mathcal{O}}^{t}, \Phi^{t}\right)
$$

which is the equivariant index of $\left.\sigma^{t}\right|_{U_{t}}$ where $U_{t}$ is a neighborhood of $\left(\Phi^{t}\right)^{-1}(\mathcal{O})$ such that $U_{t} \cap Z_{\Phi^{t}}=Z_{\mathcal{O}}^{t}$.

Let us prove that the multiplicity $\mathcal{Q}^{t}$ is independent of $t$. It is sufficient to prove that $t \rightarrow \mathcal{Q}^{t}$ is locally constant : let us show that it is constant in a neighborhood of 0 .

Note that the set $C_{\Phi^{t}}$ does not depend of $t$ : let us simply denote it by $C$. We remark that for any invariant subset $U \subset M$, we have

$$
U \cap Z_{\Phi^{t}} \subset \bigcup_{\mathcal{P} \subset \Phi^{t}(U) \cap C} Z_{\mathcal{P}}^{t}
$$

By an obvious continuity argument, we see that if $U_{0}$ is a neighborhood of $\left(\Phi^{0}\right)^{-1}(\mathcal{O})$ such that $\Phi^{0}\left(U_{0}\right) \cap C=\mathcal{O}$, then we have

$$
\Phi^{t}\left(U_{0}\right) \cap C=\mathcal{O} \quad \text { and } \quad U_{0} \cap Z_{\Phi^{t}}=Z_{\mathcal{O}}^{t}
$$

for small $t$ (says $|t| \leq \epsilon$ ). As the family $\left.\sigma^{t}\right|_{U_{0}}, t \in[0, \epsilon]$ defines an homotopy of transversally elliptic symbols, they have the same equivariant index. Finally, for small $t$, we have

$$
\begin{aligned}
\mathcal{Q}^{0}=\operatorname{Index}_{K}\left(\left.\sigma^{0}\right|_{U_{0}}\right) & =\operatorname{Index}_{K}\left(\left.\sigma^{t}\right|_{U_{0}}\right) \\
& =\mathcal{Q}^{t} .
\end{aligned}
$$

\subsection{Atiyah symbol}

Let $N$ be a Hermitian vector space. We denote by $\mathrm{U}$ the unitary group of transformations of $N$, and by $U(1) \subset \mathrm{U}$ the subgroup formed the homotheties $x \mapsto e^{i \theta} x$. Consider $S=\bigwedge \bar{N}$, the irreducible Clifford module for $N$ 
with Clifford action $\mathbf{c}(n)$. If we identify $\mathrm{T}^{*} N=N \oplus N^{*}$ with $N \oplus N$, the U-equivariant symbol $\sigma(N, S)$ is defined by

$$
\sigma(N, S)(x, \nu)=\mathbf{c}(\tilde{\nu}): \bigwedge^{\text {even }} \bar{N} \longrightarrow \bigwedge^{\text {odd }} \bar{N}, \quad(x, \nu) \in \mathrm{T}^{*} N
$$

The matrix $\beta=i \mathrm{Id}$ is an invariant element of $\operatorname{Lie}(\mathrm{U})$. We have $\beta \cdot x=J x$ where $J$ is the multiplication by $i$ on $N$. We can then define the pushed symbol $\sigma(N, S, \beta)$.

Definition 6.14 The Atiyah symbol $\operatorname{At}(N) \in \Gamma\left(\mathrm{T}^{*} N, \operatorname{hom}\left(\mathcal{S}^{+}, \mathcal{S}^{-}\right)\right)$is defined by

$$
\operatorname{At}(N)(x, \nu)=\mathbf{c}(\tilde{\nu}+J x): \bigwedge^{\text {even }} \bar{N} \longrightarrow \bigwedge^{\text {odd }} \bar{N}, \quad(x, \nu) \in \mathrm{T}^{*} N .
$$

The symbol $\operatorname{At}(N)$ is not elliptic since $\operatorname{supp}(\operatorname{At}(N))=\{(x, \nu), \tilde{\nu}+J x=$ $0\}$ is not compact. But remark that $x \rightarrow J x$ is the tangent vector produced by the action of $U(1)$ on $N$. Thus At is a $U(1)$-transversally elliptic symbol. Indeed the two equations $\tilde{\nu}+J x=0$ and $\langle\nu, J x\rangle=0$ imply $\tilde{\nu}=x=0$. So a fortiori the symbol $\operatorname{At}(N)$ is U-transversally elliptic. Let us consider $\operatorname{Sym}(N)=\oplus_{k=0}^{\infty} \operatorname{Sym}^{k}(N)$ where $\operatorname{Sym}^{k}(N)$ is the subspace of $\otimes^{k} N$ formed of symmetric tensors.

The following proposition is proved in [1] (see also [9]).

Proposition 6.15 We have $\operatorname{Index}_{\mathrm{U}}^{N}(\operatorname{At}(N))=\operatorname{Sym}(N)$ in $\hat{R}(\mathrm{U})$.

More generally, consider the case of a real vector space $N$ equipped with a linear action of a compact Lie group $K$. Let $\beta \in \mathfrak{k}$ be a $K$-invariant element such that $\mathcal{L}(\beta): N \rightarrow N$ is invertible. We denote $\mathbb{T}_{\beta} \subset K$ the closed torus contained in the center equal to the closure of $\exp (\mathbb{R} \beta)$.

We denote by $N_{J_{\beta}}$ the Hermitian vector space $N$ equipped with the invariant complex structure $J_{\beta}$. We consider the $K$-equivariant $\mathbb{T}_{\beta}$-transversally elliptic symbol $\operatorname{At}_{\beta}(N)$ defined by

$$
\operatorname{At}_{\beta}(N)(v, \xi)=\mathbf{c}(\tilde{\xi}+\beta \cdot v): \bigwedge^{\text {even }} \overline{N_{J_{\beta}}} \longrightarrow \bigwedge^{\text {odd }} \overline{N_{J_{\beta}}} .
$$

Its equivariant index satisfies

$$
\operatorname{Index}_{K}^{N}\left(\operatorname{At}_{\beta}(N)\right)=\operatorname{Sym}\left(N_{J_{\beta}}\right) .
$$


The meaning of this equation is as follows. We write $\operatorname{Sym}\left(N_{J_{\beta}}\right)$ as the sum of the finite dimensional spaces $\operatorname{Sym}^{k}\left(N_{J_{\beta}}\right)$. We consider $\operatorname{Sym}^{k}\left(N_{J_{\beta}}\right)$ as a representation space for $K$, write $\operatorname{Sym}^{k}\left(N_{J_{\beta}}\right)=\sum_{\mu \in \hat{K}} \mathrm{~m}(\mu, k) V_{\mu}$. For each irreducible representation $V_{\mu} \in \hat{K}$, the infinitesimal action of $\beta$ on $V_{\mu}$ is by a scalar $i z_{\mu} \operatorname{Id}_{V_{\mu}}$, as $\beta$ is fixed by $K$. On the other hand, the action of $\beta$ in $\operatorname{Sym}^{k}\left(N_{J_{\beta}}\right)$ is diagonalizable with eigenvalues $\sum_{J} i a_{j}$ with $J$ a subset of cardinal $k$ of $\left[1,2, \ldots, \operatorname{dim}_{\mathbb{C}} N\right]:$ here $a_{j}>0$ are the eigenvalue of $\frac{\mathcal{L}(\beta)}{i}$ on $N_{J_{\beta}}$. As $\sum_{J} a_{j}$ is larger than $k \min \left(a_{j}\right)$, we see that $k$ has to be small enough in order that $\mathrm{m}(\mu, k) \neq 0$. Thus the sum $\sum_{k} \sum_{\mu \in \hat{K}} m(\mu, k) V_{\mu}$ is a well defined element of $\hat{R}(K)$.

We now consider a $K$-equivariant Euclidean vector bundle $\mathcal{N} \rightarrow \mathcal{X}$ on a connected $K$-manifold $\mathcal{X}$. Let us assume that $\beta \in \mathfrak{k}$ is an element fixed by $K$ and acting fiberwise on $\mathcal{N}$ by an invertible transformation : in other words $\mathcal{N}^{\beta}=\mathcal{X}$. The eigenvalues of $\beta^{2}$ on the fibers are strictly negative and constant over each connected component of $\mathcal{X}$. Using Definition 5.3 of $J_{\beta}$, we define on $\mathcal{N}$ a Hermitian structure invariant by $K$. We denote by $\mathcal{N}_{J_{\beta}}$ the vector bundle $\mathcal{N}$ considered as a complex vector bundle with complex structure $J_{\beta}$. By definition of $J_{\beta}$, the list of eigenvalues of $\beta$ on $\mathcal{N}_{m}$ for the complex structure $J_{\beta}$ are $\left[i a_{1}, i a_{2}, \ldots, i a_{R}\right]$ where the $a_{j}$ are strictly positive.

We denote by $\wedge \mathcal{N}_{J_{\beta}}$ the complex exterior bundle of the complex vector bundle $\mathcal{N}_{J_{\beta}}$. We denote by $\operatorname{Sym}\left(\mathcal{N}_{J_{\beta}}\right)$ the $\mathbb{Z}$-graded symmetric vector bundle of $\mathcal{N}_{J_{\beta}}$.

Let $N$ be the fiber $\left.\mathcal{N}\right|_{x_{o}}$ at some point $x_{o} \in \mathcal{X}$, equipped with the linear action of $\beta$, the complex structure $J_{\beta}$ and its Euclidean structure. Let $\mathrm{U}$ be the subgroup of $\mathrm{U}(N)$ of elements that commutes with $\mathcal{L}(\beta)$. Let $P_{U} \rightarrow \mathcal{X}$ be the U-unitary framed bundle of $\mathcal{N}$ : for $x \in \mathcal{X}$, we take

$$
\left.P_{U}\right|_{x}:=\left\{f:\left.N \rightarrow \mathcal{N}\right|_{x}, \text { unitary and } \mathcal{L}(\beta) \text { - equivariant }\right\} .
$$

Hence the vector bundle $\mathcal{N}$ is equal to $P \times_{\mathrm{U}} N$. The Atiyah symbol on $N$ defines a class

$$
\operatorname{At}_{\beta}(N) \in \mathbf{K}_{\mathrm{U} \times \mathbb{T}_{\beta}}^{0}\left(\mathrm{~T}_{\mathbb{T}_{\beta}}^{*} N\right) .
$$

Let us use the notion of fiber product introduced in Section 4.4: here we work with the group $S:=\mathbb{T}_{\beta}$. We can define the morphism $\operatorname{At}_{\beta}$ : $\mathbf{K}_{K}^{0}\left(\mathrm{~T}_{K}^{*} \mathcal{X}\right) \longrightarrow \mathbf{K}_{K}^{0}\left(\mathrm{~T}_{K}^{*} \mathcal{N}\right)$ by the relation

$$
\operatorname{At}_{\beta}(\sigma):=\sigma \diamond \operatorname{At}_{\beta}(N)
$$

where $\sigma$ is a $K$-transversally elliptic symbol on $\mathcal{X}$. 
Lemma 4.6 gives

$$
\begin{aligned}
\operatorname{Index}_{K}^{\mathcal{N}}\left(\operatorname{At}_{\beta}(\sigma)\right) & =\operatorname{Index}_{K}^{\mathcal{X}}\left(\sigma \otimes \operatorname{Sym}\left(\mathcal{N}_{J_{\beta}}\right)\right) \\
& =\sum_{k=0}^{\infty} \operatorname{Index}_{K}^{\mathcal{X}}\left(\sigma \otimes \operatorname{Sym}^{k}\left(\mathcal{N}_{J_{\beta}}\right)\right) .
\end{aligned}
$$

A symbol representing $\operatorname{At}_{\beta}(\sigma)$ is as follows. We write an element $n \in \mathcal{N}$ as $(x, v)$ with $x \in \mathcal{X}$ and $\left.v \in \mathcal{N}\right|_{x}$. We choose a $K$-invariant connection on the bundle $\mathcal{N} \rightarrow \mathcal{X}$, which allows us to parameterize $\mathrm{T}^{*} \mathcal{N}=\{(n,(\xi, \nu))\}$, with $\xi \in \mathrm{T}_{x}^{*} \mathcal{X},\left.\nu \in \mathcal{N}^{*}\right|_{x}$ (here $\xi$ is lifted to an element of $\mathrm{T}_{n}^{*} \mathcal{N}$ using the connection). Let $\sigma(x, \xi) \in \Gamma\left(\mathrm{T}^{*} \mathcal{X}, \operatorname{hom}\left(\mathcal{E}^{+}, \mathcal{E}^{-}\right)\right)$be a symbol on $\mathcal{X}$. Then

$$
\operatorname{At}_{\beta}(\sigma)((x, v),(\xi, \nu))=\sigma(x, \xi) \otimes \mathbf{c}_{x}(\tilde{\nu}+\beta v) .
$$

Here $\mathbf{c}_{x}$ is the Clifford action on $\left.\bigwedge \overline{\mathcal{N}}_{J_{\beta}}\right|_{x}$, so $\mathbf{c}_{x}(\tilde{\nu}+\beta v)^{2}=-\|\tilde{\nu}+\beta v\|^{2} \mathrm{Id}$. We see that $\operatorname{Char}\left(\operatorname{At}_{\beta}(\sigma)\right) \cap \mathrm{T}_{K}^{*} \mathcal{N}=\operatorname{Char}(\sigma) \cap \mathrm{T}_{K}^{*} \mathcal{X}$.

\section{Abelian Localization formula}

\subsection{Atiyah-Segal-Singer localization formula}

Let $M$ be a compact $K$-manifold. Let $\beta \in \mathfrak{k}$ be a central element, and let $M^{\beta}$ be the submanifold of points fixed by the infinitesimal action of $\beta$. Let $\mathcal{N}$ be the normal bundle of $M^{\beta}$ in $M$. Then $\beta$ acts by a fiberwise invertible transformation on $\mathcal{N}$. Denote by $\mathcal{N}_{J_{\beta}}$ the (complex) vector bundle $\mathcal{N}$ polarized by $\beta$.

Let $\mathcal{U}$ be a tubular neighborhood of $M^{\beta}$ in $M$ that is diffeomorphic with the normal bundle $\mathcal{N}$. We denote by $\varphi: \mathcal{U} \rightarrow \mathcal{N}$ the $K$-diffeomorphism and by $i: \mathcal{U} \hookrightarrow M$ the inclusion. Let $\varphi^{*}: \mathbf{K}_{K}^{0}\left(\mathrm{~T}_{K}^{*} \mathcal{N}\right) \rightarrow \mathbf{K}_{K}^{0}\left(\mathrm{~T}_{K}^{*} \mathcal{U}\right)$ and $i_{*}: \mathbf{K}_{K}^{0}\left(\mathrm{~T}_{K}^{*} \mathcal{U}\right) \rightarrow \mathbf{K}_{K}^{0}\left(\mathrm{~T}_{K}^{*} M\right)$ the corresponding maps in $\mathbf{K}$-theory.

We denote by

$$
\mathbf{A t}_{\beta}: \mathbf{K}_{K}^{0}\left(\mathrm{~T}_{K}^{*} M^{\beta}\right) \longrightarrow \mathbf{K}_{K}^{0}\left(\mathrm{~T}_{K}^{*} M\right)
$$

the map $i_{*} \circ \varphi^{*} \circ \mathrm{At}_{\beta}$. For the equivariant index, we have the equality

$$
\operatorname{Index}_{K}^{M}\left(\operatorname{At}_{\beta}(\sigma)\right)=\operatorname{Index}_{K}^{M^{\beta}}\left(\sigma \otimes \operatorname{Sym}\left(\mathcal{N}_{J_{\beta}}\right)\right)
$$

for any $\sigma \in \mathbf{K}_{K}^{0}\left(\mathrm{~T}_{K}^{*} M^{\beta}\right)$.

Let $\mathcal{E} \rightarrow M$ be a (graded) Clifford module on $M$. The bundle $\bigwedge \overline{\mathcal{N}_{J_{\beta}}}$ is a $\operatorname{Spin}^{c}$ bundle for the Euclidean bundle $\mathcal{N}$. 
Definition 7.1 There exists a unique graded Clifford bundle $\mathbb{d}_{\beta}(\mathcal{E})$ over the tangent bundle $\mathrm{T} M^{\beta}$, such that, when we restrict $\mathcal{E}$ to $M^{\beta}$, we get the isomorphism

$$
\left.\mathcal{E}\right|_{M^{\beta}} \simeq \mathbb{d}_{\beta}(\mathcal{E}) \otimes \bigwedge \overline{\mathcal{N}_{J_{\beta}}}
$$

of graded Clifford bundles relative to the decomposition $\left.\mathrm{T} M\right|_{M^{\beta}}:=\mathrm{T} M^{\beta} \oplus$ $\mathcal{N}$.

We have two elliptic symbols : $\sigma(M, \mathcal{E}) \in \mathbf{K}_{K}^{0}\left(\mathrm{~T}^{*} M\right)$ and $\sigma\left(M^{\beta}, \mathbb{d}_{\beta}(\mathcal{E})\right) \in$ $\mathbf{K}_{K}^{0}\left(\mathrm{~T}^{*} M^{\beta}\right)$.

Proposition 7.2 (Atiyah-Segal-Singer localization formula) The following relation holds in $\mathbf{K}_{K}^{0}\left(\mathrm{~T}_{K}^{*} M\right)$ :

$$
\mathbf{A t}_{\beta}\left(\sigma\left(M^{\beta}, \mathbb{d}_{\beta}(\mathcal{E})\right)\right)=\sigma(M, \mathcal{E}) .
$$

In particular $\mathcal{Q}_{K}(M, \mathcal{E})=\mathcal{Q}_{K}\left(M^{\beta}, \mathbb{d}_{\beta}(\mathcal{E}) \otimes \operatorname{Sym}\left(\mathcal{N}_{J_{\beta}}\right)\right)$.

Remark 7.3 In Atiyah-Segal, this equation is proved in the tensor product of $\mathbf{K}_{K}^{0}\left(\mathrm{~T}^{*} M\right)$ with the field of fractions of $R(K)$. In [1], this more precise relation is proved in $\mathbf{K}_{K}^{0}\left(\mathrm{~T}_{K}^{*} M\right)$.

Proof. We deform the symbol $\sigma(M, \mathcal{E})$ by the vector field $\beta_{M}$. So we apply the deformation introduced in Definition 6.10 with the constant map $\Phi=\beta$. The pushed-symbol $\sigma(M, \mathcal{E}, \beta)$ is

$$
\sigma(M, \mathcal{E}, \beta)(m, \nu):=\mathbf{c}_{\mathcal{E}_{m}}(\tilde{\nu}+\beta \cdot m): \mathcal{E}_{m}^{+} \rightarrow \mathcal{E}_{m}^{-} .
$$

The excision property tells us that $\sigma(M, \mathcal{E})=i_{*}\left(\left.\sigma(M, \mathcal{E}, \beta)\right|_{\mathcal{U}}\right)$ in $\mathbf{K}_{K}^{0}\left(\mathrm{~T}_{K}^{*} M\right)$. We now analyze the symbol $\tilde{\sigma}:=\left(\varphi^{-1}\right)^{*}(\sigma(M, \mathcal{E}, \beta) \mid \mathcal{U})$ on $\mathcal{N}$. Let $\pi: \mathcal{N} \rightarrow$ $M^{\beta}$ be the projection. Over $\mathcal{N}$, the Clifford bundle $\tilde{\mathcal{E}}:=\left(\varphi^{-1}\right)^{*}\left(\left.\mathcal{E}\right|_{\mathcal{U}}\right)$ is isomorphic to $\pi^{*}\left(\mathbb{d}_{\beta}(\mathcal{E})\right) \otimes \pi^{*}\left(\bigwedge \overline{\mathcal{N}_{J_{\beta}}}\right)$. With notations of Subsection 5.3, we may assume that for $(n, \nu) \in \mathrm{T}^{*} \mathcal{N}$, and $n=(x, v)$, the map $\tilde{\sigma}(n,(\xi, \nu))$ is the exterior product of the map

$$
\mathbf{c}_{x}^{1}(\tilde{\xi}):\left.\left.\mathbb{d}_{\beta}(\mathcal{E})\right|_{x} ^{+} \longmapsto \mathbb{d}_{\beta}(\mathcal{E})\right|_{x} ^{-}
$$

with the map

$$
\mathbf{c}_{x}^{2}(\tilde{\nu}+\beta \cdot v):\left.\left.\bigwedge^{\text {even }} \overline{\mathcal{N}_{J_{\beta}}}\right|_{x} \longmapsto \bigwedge^{\text {odd }} \overline{\mathcal{N}_{J_{\beta}}}\right|_{x} .
$$

Here $\xi$ is viewed as a horizontal vector in $\mathrm{T}_{n}^{*} \mathcal{N}$ and $\tilde{\nu}, \beta \cdot v$ are vertical vectors in $\mathrm{T}_{n} \mathcal{N}$. By definition, this means that $\tilde{\sigma}$ is equal to $\sigma\left(M^{\beta}, \mathbb{d}_{\beta}(\mathcal{E})\right) \diamond \operatorname{At}_{\beta}(N)$. 


\subsection{Deformed Atiyah-Segal-Singer localization formula}

We now prove a slightly more general version of the preceding Atiyah-SegalSinger localization formula.

Let $\mathcal{N} \rightarrow \mathcal{X}$ be a $K$-equivariant vector bundle over $\mathcal{X}$, and let $\beta$ be a central element in $\mathfrak{k}$ such that $\mathcal{X}=\mathcal{N}^{\beta}$. Thus $\beta$ acts invertibly on the vector bundle $\mathcal{N}$. Let $\Phi: \mathcal{N} \rightarrow \mathfrak{k}^{*}$ be a $K$-invariant map and $Z_{\Phi} \subset \mathcal{N}$ be the set of zeroes of the associated Kirwan vector field $\kappa_{\Phi}$. We do not assume the basis $\mathcal{X}$ compact, but we assume that $\Phi_{\mathcal{X}}:=\left.\Phi\right|_{\mathcal{X}}: \mathcal{X} \rightarrow \mathfrak{k}^{*}$ is a proper map.

The intersection of the set $Z_{\Phi}$ with $\mathcal{X}$ is equal to $Z_{\Phi_{\mathcal{X}}}$.

Lemma 7.4 Assume that $Z_{\Phi} \cap \mathcal{X}=\Phi_{\mathcal{X}}^{-1}(\beta)$. Then $\Phi_{\mathcal{X}}^{-1}(\beta)$ is a compact component of $Z_{\Phi}$ : there exists a $K$-invariant neighborhood $\mathcal{U}$ of $\Phi_{\mathcal{X}}^{-1}(\beta)$ in $\mathcal{N}$ such that $Z_{\Phi} \cap \mathcal{U}=\Phi_{\mathcal{X}}^{-1}(\beta)$.

Proof. Consider a $K$-invariant decomposition of the tangent bundle $\mathrm{T} \mathcal{N}=H \oplus V$ in vertical and horizontal tangent bundles. Here $V=\pi^{*} \mathcal{N}$. As $\mathcal{N}$ is a $K$-equivariant vector bundle, for $X \in \mathfrak{k}$, and $n=(x, v) \in \mathcal{N}$, the vertical projection $\left.\left[X_{\mathcal{N}}\right]^{V}(x, v) \in \mathcal{N}\right|_{x}$ of the vector field $X_{\mathcal{N}}$ depends linearly of $v$. In other words, there exists $\mu(X) \in \Gamma(\mathcal{X}, \operatorname{End}(\mathcal{N}))$ such that $\left[X_{\mathcal{N}}\right]^{V}(x, v)=\left.\mu(X)_{x} \cdot v \in \mathcal{N}\right|_{x}$, where $x \in \mathcal{X}, v \in \mathcal{N}_{x}$.

For $X=\beta,-\mu(\beta)$ is equal to the linear action $\mathcal{L}(\beta)$ of $\beta$ on $\mathcal{N}_{x}$. If $\mathcal{K} \subset \mathcal{X}$ is a compact subset, there exists $c_{\mathcal{K}}>0$ such that $\left\|\left[X_{\mathcal{N}}\right]^{V}(x, v)\right\| \leq$ $c_{\mathcal{K}}\|X\|\|v\|$ for all $(x, v) \in \pi^{-1}(\mathcal{K})$.

The Kirwan vector field $\kappa_{\Phi}(n)=-\Phi(n) \cdot n$ admits then the decomposition $\kappa_{\Phi}=\left[\kappa_{\Phi}\right]^{V} \oplus\left[\kappa_{\Phi}\right]^{H}$ with $\left[\kappa_{\Phi}\right]^{V}(n)=-\left.\mu(\Phi(n))_{x} \cdot v \in \mathcal{N}\right|_{x}$.

Choose a relatively compact open neighborhood $\mathcal{V}$ of $\Phi_{\mathcal{X}}^{-1}(\beta)$ in $\mathcal{X}$, and consider $\mathcal{U}_{\epsilon}:=\left\{n \in \pi^{-1}(\mathcal{V}) ;\|\Phi(n)-\beta\|<\epsilon\right\}$ which is an open neighborhood of $\Phi_{\mathcal{X}}^{-1}(\beta)$ in $\mathcal{N}$.

If $R(n)=\Phi(n)-\beta$, we have

$$
\mu(\Phi(n))_{x} \cdot v=\beta \cdot v+\mu(R(n))_{x} \cdot v .
$$

We choose $\epsilon$ sufficiently small so that $\left\|\mu(R(n))_{x} \cdot v\right\|<\|\beta \cdot v\|$ for any $n=(x, v) \in \mathcal{U}_{\epsilon}$. If $\kappa_{\Phi}(n)=0$, taking the scalar product with the vertical vector $\beta \cdot v$, we obtain

$$
0=-\left\langle\kappa_{\Phi}(n), \beta \cdot v\right\rangle=-\left\langle\left[\kappa_{\Phi}\right]^{V}(n), \beta \cdot v\right\rangle=\|\beta \cdot v\|^{2}+\left\langle\mu(R(n))_{x} \cdot v, \beta \cdot v\right\rangle
$$

and this implies $v=0$. Thus the set of zeroes of $\kappa_{\Phi}$ on $\mathcal{U}_{\epsilon}$ is the set of zeroes of $\kappa_{\Phi}$ on $\mathcal{X}$. 
Let $\mathcal{E} \rightarrow \mathcal{N}$ be a graded Clifford bundle on the manifold $\mathcal{N}$. We can then define the $K$-transversally elliptic class $\sigma(\mathcal{N}, \mathcal{E}, Z, \Phi) \in \mathbf{K}_{K}^{0}\left(\mathrm{~T}_{K}^{*} \mathcal{N}\right)$ attached to the compact component $Z=\Phi_{\mathcal{X}}^{-1}(\beta)$.

Let $\mathbb{d}_{\beta}(\mathcal{E})$ be the graded Clifford bundle on $\mathcal{X}$ induced by $\mathcal{E}$ (see Definition 7.1). We can also define the $K$-transversally elliptic class $\sigma\left(\mathcal{X}, \mathbb{d}_{\beta}(\mathcal{E}), Z, \Phi_{\mathcal{X}}\right) \in \mathbf{K}_{K}^{0}\left(\mathrm{~T}_{K}^{*} \mathcal{X}\right)$.

Proposition 7.5 (Variation on Atiyah-Segal localization formula) The following relation holds in $\mathbf{K}_{K}^{0}\left(\mathrm{~T}_{K}^{*} \mathcal{N}\right)$ :

$$
\operatorname{At}_{\beta}\left(\sigma\left(\mathcal{X}, \mathbb{d}_{\beta}(\mathcal{E}), Z, \Phi_{\mathcal{X}}\right)\right)=\sigma(\mathcal{N}, \mathcal{E}, Z, \Phi) .
$$

In particular $\mathcal{Q}_{K}(\mathcal{N}, \mathcal{E}, Z, \Phi)=\mathcal{Q}_{K}\left(\mathcal{X}, \mathbb{d}_{\beta}(\mathcal{E}) \otimes \operatorname{Sym}\left(\mathcal{N}_{J_{\beta}}\right), Z, \Phi_{\mathcal{X}}\right)$.

Proof. The proof is a variation of the proof of Proposition 7.2. We consider the vector field $\kappa^{0}(n)=-\beta \cdot n \oplus \kappa_{\mathcal{X}}(x)$ where $\kappa_{\mathcal{X}}(x) \in T_{x} \mathcal{X}$ is lifted horizontally in $\mathrm{T} \mathcal{N}=\pi^{*} \mathrm{~T} \mathcal{X} \oplus \pi^{*} \mathcal{N}$. Then $\kappa^{t}=t \kappa^{0}+(1-t) \kappa_{\Phi}$ has vertical component $-\beta \cdot v+(1-t) \mu(R(n))_{x} \cdot v$. The restriction to $\mathcal{X}$ of $\kappa^{t}$ is the vector field $\kappa_{\Phi_{\mathcal{X}}}$ for any $t \in[0,1]$. We then consider

$$
\sigma^{t}(n, \nu)=\mathbf{c}_{n}\left(\tilde{\nu}-\kappa^{t}(n)\right)
$$

for $t \in[0,1]$. We see, as before, that $\operatorname{Char}\left(\sigma^{t}\right)$ intersected with $\mathrm{T}_{K}^{*}(\mathcal{N})$ remains equal to $Z$. Thus the class of $\sigma^{t}$ is constant in $\mathbf{K}_{K}^{0}\left(\mathrm{~T}_{K}^{*} \mathcal{N}\right)$, and $\sigma^{0}$ is equal to $\operatorname{At}_{\beta}\left(\sigma\left(\mathcal{X}, \mathbb{d}_{\beta}(\mathcal{E}), Z, \Phi_{\mathcal{X}}\right)\right)$.

\section{Non abelian localization formula}

In this section, given an equivariant map $\Phi: M \rightarrow \mathfrak{k}^{*}$, we establish a general localization formula for the equivariant index of a Dirac operator.

\subsection{Localization on $\Phi^{-1}(0)$}

Let $\Phi: M \rightarrow \mathfrak{k}^{*}$ be an equivariant proper map. We describe what is the representation $\mathcal{Q}_{K}\left(M, \mathcal{E}, \Phi^{-1}(0), \Phi\right)$, in the special case where 0 is a regular value of $\Phi$, and where $K$ acts infinitesimally freely on the compact submanifold $P=\Phi^{-1}(0)$. Let $M_{\text {red }}:=P / K$ be the corresponding "reduced" space, and let $\pi: P \rightarrow M_{\text {red }}$ be the projection map.

Remark 8.1 By Proposition 6.6, the hypothesis that 0 is a regular value implies that $K$ acts infinitesimally freely on $P$ in the case where $\Phi$ is a moment map. 
On $P$, we obtain an exact sequence $\left.0 \longrightarrow \mathrm{T} P \longrightarrow \mathrm{T} M\right|_{P} \stackrel{\mathrm{T} \Phi}{\longrightarrow}\left[\mathfrak{k}^{*}\right] \rightarrow$ 0 , where $\left[\mathfrak{k}^{*}\right]$ is the trivial bundle $P \times \mathfrak{k}^{*}$. We have also an orthogonal decomposition $\mathrm{T} P=\mathrm{T}_{K} P \oplus[\mathfrak{k}]$ where $[\mathfrak{k}]$ is the subbundle identified to $P \times \mathfrak{k}$ through the map $(p, X) \mapsto X \cdot p$. So TM $\left.\right|_{P}$ admits the orthogonal decomposition $\left.\mathrm{T} M\right|_{P} \simeq \mathrm{T}_{K} P \oplus[\mathfrak{k}] \oplus\left[\mathfrak{k}^{*}\right]$. We rewrite this as

$$
\left.\mathrm{T} M\right|_{P} \simeq \mathrm{T}_{K} P \oplus\left[\mathfrak{k}_{\mathbb{C}}\right]
$$

with the convention $[\mathfrak{k}]=P \times(\mathfrak{k} \otimes \mathbb{R})$ and $\left[\mathfrak{k}^{*}\right]=P \times(\mathfrak{k} \otimes i \mathbb{R})$. Note that the bundle $\mathrm{T}_{K} P$ is naturally identified with $\pi^{*}\left(\mathrm{~T} M_{\text {red }}\right)$.

Following Definition 7.1, we can divide the graded Clifford bundle $\left.\mathcal{E}\right|_{P}$ by the $\operatorname{Spin}^{c}$-bundle $\Lambda \mathfrak{k}_{\mathbb{C}}$ for the vector space $\mathfrak{k}_{\mathbb{C}}$.

Definition 8.2 Let $\mathcal{E}_{\text {red }}$ be the graded Clifford bundle on the vector bundle $\mathrm{T}_{K} P \rightarrow P$ such that

$$
\left.\mathcal{E}\right|_{P} \simeq \mathcal{E}_{\text {red }} \otimes\left[\bigwedge \mathfrak{k}_{\mathbb{C}}\right]
$$

is an isomorphism of graded Clifford bundles on $\left.\mathrm{T} M\right|_{P}$. We still denote by $\mathcal{E}_{\text {red }}$ the induced Clifford (orbi-)bundle on $\mathrm{T} M_{\text {red }}$.

For any representation space $V_{\mu}$ of $K$, we can form the twisted Clifford bundle $\mathcal{E}_{\text {red }} \otimes \mathcal{V}_{\mu}^{*}$ of the Clifford bundle $\mathcal{E}_{\text {red }}$ by the equivariant (orbi)-bundle $\mathcal{V}_{\mu}^{*}=P \times_{K} V_{\mu}^{*}$.

Consider the horizontal Dirac symbol, $\sigma_{\text {hor }}\left(P, \mathcal{E}_{\text {red }}\right)$ on $P$ defined in Section 5.6. This is a $K$ transversally elliptic symbol on $P$. We now prove

Theorem 8.3 If 0 is a regular value of $\Phi$ and $K$ acts locally freely on $\Phi^{-1}(0)$, then $\Phi^{-1}(0)$ is a component of $Z_{\Phi}$, and we have

$$
\begin{aligned}
\mathcal{Q}_{K}\left(M, \mathcal{E}, \Phi^{-1}(0), \Phi\right) & =\operatorname{Index}_{K}^{P}\left(\sigma_{\text {hor }}\left(P, \mathcal{E}_{\text {red }}\right)\right) \\
& =\sum_{\mu \in \hat{K}} \mathcal{Q}\left(M_{\text {red }}, \mathcal{E}_{\text {red }} \otimes \mathcal{V}_{\mu}^{*}\right) V_{\mu} .
\end{aligned}
$$

In particular we have $\left[\mathcal{Q}_{K}\left(M, \mathcal{E}, \Phi^{-1}(0), \Phi\right)\right]^{K}=\mathcal{Q}\left(M_{\text {red }}, \mathcal{E}_{\text {red }}\right)$.

Proof. Let us study the pushed symbol $\sigma(M, \mathcal{E}, \Phi)(m, \nu)=\sigma_{\mathcal{E}_{m}}(\tilde{\nu}-$ $\left.\kappa_{\Phi}(m)\right)$ when $m$ is in a neighborhood of $P=\Phi^{-1}(0)$. So we can assume that $M=P \times \mathfrak{k}^{*}$. For a point $m=(x, \xi) \in P \times \mathfrak{k}^{*}$, the tangent vector $\tilde{\nu} \in \mathrm{T}_{m} M$ is represented by $\eta \oplus a \oplus b$ : here $\eta \in \mathrm{T}_{K} P, a \in \mathfrak{k}$ represents the tangent vector $a \cdot x \in \mathrm{T}_{x} P$, and $b=\mathrm{T}_{m} \Phi(\tilde{\nu}) \in \mathfrak{k}^{*}$. For $X \in \mathfrak{k}$, the vector field $X_{M}(x, \xi)$ associated to the action of $K$ on $P \times \mathfrak{k}^{*}$ is represented by $0 \oplus-X \oplus[\xi, X]$ : 
in particular the Kirwan vector field $\kappa_{\Phi}(x, \xi)$ is equal to $0 \oplus-\xi \oplus 0$. For the pushed symbol we obtain

$$
\sigma(M, \mathcal{E}, \Phi)(m, \nu)=\mathbf{c}_{\mathcal{E}_{r e d}}(\eta) \otimes \mathbf{c}_{\wedge \mathfrak{k}_{\mathbb{C}}}((a+\xi) \oplus i b) .
$$

Let us see that the support of the pushed symbol intersected with $\mathrm{T}_{K}^{*} M$ is just equal to $P$. Indeed if $(x, \xi ; \eta \oplus a \oplus b) \in \mathrm{T}_{K}^{*} M$, we have $([\xi, X], b)-$ $(X, a)=0$ for all $X \in \mathfrak{k}$, e.g. $a=[b, \xi]$. Then, if $\mathbf{c}_{\mathcal{E}_{x}}(\eta) \otimes \mathbf{c}_{\wedge \mathfrak{k}_{\mathbb{C}}}((a+\xi) \oplus i b)$ is not invertible, we obtain $\eta=0, b=0$ and $a+\xi=0$. If furthermore $(x, \xi ; \eta \oplus a \oplus b)$ is in $\mathrm{T}_{K}^{*} M$, we obtain $a=\xi=0$. Thus we see that $\Phi^{-1}(0)=P$ is a component of $Z_{\Phi}$, and $\sigma(M, \mathcal{E}, \Phi)$ restricted to a neighborhood of $P$ defines a transversally elliptic symbol on $M$. We can then define its index $\mathcal{Q}_{K}\left(M, \mathcal{E}, \Phi^{-1}(0), \Phi\right)$.

Consider now the injection $i: P \rightarrow P \times \mathfrak{k}^{*}=M$ and the horizontal symbol $\sigma_{h o r}\left(P, \mathcal{E}_{\text {red }}\right)$ on $P$. By definition (see Section 5.4), the direct image $i_{1} \sigma_{\text {hor }}\left(P, \mathcal{E}_{\text {red }}\right)$ is defined to be the restriction to $\mathrm{T}_{K}^{*} M$ of the symbol

$$
\mathbf{c}_{\mathcal{E}_{\text {red }}}(\eta) \otimes \mathbf{c}_{\wedge \mathfrak{k}_{\mathbb{C}}}(\xi \oplus i b) .
$$

We see that for $t \in[0,1]$, the support of the symbol

$$
\sigma^{t}(x, \xi, \nu)=\mathbf{c}_{\mathcal{E}_{\text {red }}}(\eta) \otimes \mathbf{c}_{\wedge \mathfrak{k}_{\mathbb{C}}}((t a+\xi) \oplus i b)
$$

intersected with $\mathrm{T}_{K}^{*} M$ remains equal to $P$.

Thus $\sigma(M, \mathcal{E}, \Phi)$ defines the same class in $K$-theory than the symbol $i_{!} \sigma_{h o r}\left(P, \mathcal{E}_{\text {red }}\right)$. Thus by the direct image property of the symbol, we obtain our result.

If furthermore we had an action of $G \times K$ on $M$, we would obtain an action of $G$ in each space $\mathcal{Q}\left(M_{\text {red }}, \mathcal{E}_{\text {red }} \otimes \mathcal{V}_{\mu}^{*}\right)$ and the $G \times K$ decomposition

$$
\mathcal{Q}_{G \times K}\left(M, \mathcal{E}, \Phi^{-1}(0), \Phi\right)=\sum_{\mu \in \hat{K}} \mathcal{Q}_{G}\left(M_{r e d}, \mathcal{E}_{r e d} \otimes \mathcal{V}_{\mu}^{*}\right) \otimes V_{\mu}
$$

\subsection{Coadjoint orbits and slices}

Let $\Phi: M \rightarrow \mathfrak{k}^{*}$ be our $K$-equivariant map (with $K$ not necessarily connected).

Consider the coadjoint action of $K$ on $\mathfrak{k}^{*}$. Let $\xi \in \mathfrak{k}^{*}$. Let us consider the $K_{\xi}$-invariant decomposition $\mathfrak{k}=\mathfrak{k}_{\xi} \oplus \mathfrak{q}$, with dual decomposition $\mathfrak{k}^{*}=\mathfrak{k}_{\xi}^{*} \oplus \mathfrak{q}^{*}$.

Proposition 8.4 Let $\xi \in \mathfrak{k}^{*}$, and let $B$ be a sufficiently small $K_{\xi}$-invariant neighborhood of $\xi$ in $\mathfrak{k}_{\xi}^{*}$. Then 
- $Y=\Phi^{-1}(B)$ is a $K_{\xi}$-invariant submanifold of $M$ (perhaps empty).

- $\Phi^{-1}(K B)$ is an open neighborhood of $\Phi^{-1}(K \xi)$, and is diffeomorphic to $K \times_{K_{\xi}} Y$.

We will call the manifold $Y$ a slice of $M$ at $\xi$.

Proof. Consider the coadjoint orbit $K \xi$. The map $X \rightarrow X \cdot \xi$ is a surjective map from $\mathfrak{k}$ to $\mathfrak{q}^{*}$ and $\mathfrak{k}_{\xi}^{*}$ identifies to the normal space to the coadjoint orbit $K \xi$ at $\xi$. The tubular neighborhood theorem implies that if $B$ is sufficiently small, then $K B$ is a neighborhood of $K \xi$ isomorphic to $K \times_{K_{\xi}} B$.

Write $\Phi_{\mathfrak{q}}$ for the component of $\Phi$ in $\mathfrak{q}^{*}$. The differential map $T_{y} \Phi_{\mathfrak{q}}$ : $\mathrm{T}_{y} M \rightarrow \mathfrak{q}^{*}$ is surjective, if $\Phi(y)=\xi$. Thus it is surjective at any point $y \in Y$, if $B$ is sufficiently small. The rest of the proof is standard.

We also consider $\xi$ as an element of $\mathfrak{k}_{\xi}$, using our $K$-invariant identification $\mathfrak{k}=\mathfrak{k}^{*}$. So $\xi$ induces an invertible skew-symmetric transformation on $\mathfrak{q}$. Denote by $\mathfrak{q}_{J_{\xi}}$ the vector space $\mathfrak{q}=\mathfrak{k} / \mathfrak{k}_{\xi}$ equipped with the complex structure $J_{\xi}$,

Suppose now that $M$ carries a graded Clifford bundle $\mathcal{E}$. The tangent bundle $\mathrm{T} M$, when restricted to the slice $Y$, decomposes as $\left.\mathrm{T} M\right|_{Y} \simeq \mathrm{T} Y \oplus$ $\left[\mathfrak{k} / \mathfrak{k}_{\xi}\right]$, where $\left[\mathfrak{k} / \mathfrak{k}_{\xi}\right]$ denotes the trivial bundle $\mathfrak{k} / \mathfrak{k}_{\xi} \times Y$.

As in Definition 7.1, we can divide the graded Clifford bundle $\left.\mathcal{E}\right|_{Y}$ by the Spin ${ }^{c}$-bundle $\wedge \mathfrak{q}_{J_{\xi}}$ for the Euclidean vector space $\mathfrak{q}$. There exists a unique graded Clifford bundle $\mathcal{E}_{d Y}$ over the tangent bundle $\mathrm{T} Y$, such that, when we restrict $\mathcal{E}$ to $Y$, we get the isomorphism

$$
\left.\mathcal{E}\right|_{Y} \simeq \mathcal{E}_{d Y} \otimes\left[\bigwedge \mathfrak{q}_{J_{\xi}}\right]
$$

of graded Clifford bundles relative to the decomposition $\left.\mathrm{T} M\right|_{Y} \simeq \mathrm{T} Y \oplus\left[\mathfrak{k} / \mathfrak{k}_{\xi}\right]$.

\subsection{Localization on $Z_{\beta}$}

We consider our equivariant map $\Phi: M \rightarrow \mathfrak{k}^{*}$, and we assume that

$$
Z_{\beta}=K\left(M^{\beta} \cap \Phi^{-1}(\beta)\right)
$$

is a compact component of $Z_{\Phi}$. Here $\beta$ is a non zero element in $\mathfrak{k}^{*}$.

Let $\mathcal{Q}_{K}\left(M, \mathcal{E}, Z_{\beta}, \Phi\right) \in \hat{R}(K)$ be the localized equivariant index attached to an equivariant graded Clifford bundle $\mathcal{E}$. Let $\mathbb{d}_{\beta}(\mathcal{E})$ be the induced graded Clifford bundle on $M^{\beta}$ (see Section 7.1). The restriction of $\Phi$ to $M^{\beta}$ is a 
map that takes value in $\mathfrak{k}_{\beta}^{*}$ : for simplicity we still denote $\Phi: M^{\beta} \rightarrow \mathfrak{k}_{\beta}^{*}$. The set $M^{\beta} \cap \Phi^{-1}(\beta)$ is a compact component of $Z_{\Phi} \cap M^{\beta}$. Let $\mathcal{N}_{J_{\beta}}$ be the (complex) normal bundle of $M^{\beta}$ in $M$ polarized by $\beta$. The aim of this subsection is to prove the following localization formula.

Theorem 8.5 We have the following relation in $\hat{R}(K)$ :

$$
\begin{aligned}
& \mathcal{Q}_{K}\left(M, \mathcal{E}, Z_{\beta}, \Phi\right)= \\
& \quad \operatorname{Ind}_{K_{\beta}}^{K}\left(\mathcal{Q}_{K_{\beta}}\left(M^{\beta}, \mathbb{d}_{\beta}(\mathcal{E}) \otimes \operatorname{Sym}\left(\mathcal{N}_{J_{\beta}}\right), M^{\beta} \cap \Phi^{-1}(\beta), \Phi\right) \otimes \bigwedge\left(\mathfrak{k} / \mathfrak{k}_{\beta} \otimes \mathbb{C}\right)\right) .
\end{aligned}
$$

Proof. The proof will have two steps.

First Step : Let $B \subset \mathfrak{k}^{*}$ be a small $K_{\beta}$-invariant ball around $\beta$ in $\mathfrak{k}_{\beta}^{*}$ and let $Y=\Phi^{-1}(B)$ be the corresponding slice. A neighborhood of $Z_{\beta}$ in $M$ is then diffeomorphic with $K \times_{K_{\beta}} Y$. We denote by $\Phi_{Y}: Y \rightarrow \mathfrak{k}_{\beta}^{*}$ the restriction of $\Phi$ to the submanifold $Y$. Let $\mathcal{E}_{d Y}$ be the induced Clifford bundle on $\mathrm{T} Y$ (see (8.27)).

The restriction of the symbol $\sigma(M, \mathcal{E}, \Phi)$ to $\mathrm{T}^{*} Y$ is equal to $\sigma\left(Y, \mathcal{E}_{d Y}, \Phi_{Y}\right) \otimes$ $\bigwedge \mathfrak{q}_{J_{\beta}}$, hence the induction formula of Proposition 4.7 gives that

$$
\mathcal{Q}_{K}\left(M, \mathcal{E}, Z_{\beta}, \Phi\right)=\operatorname{Ind}_{K_{\beta}}^{K}\left(\mathcal{Q}_{K_{\beta}}\left(Y, \mathcal{E}_{d Y}, M^{\beta} \cap \Phi^{-1}(\beta), \Phi_{Y}\right) \otimes \bigwedge \mathfrak{q}_{J_{\beta}}\right)
$$

(as $\Phi^{-1}(\beta)$ is contained in $Y, M^{\beta} \cap \Phi^{-1}(\beta)=Y^{\beta} \cap \Phi_{Y}^{-1}(\beta)$ ).

Second Step : Now we use the variation of the Atiyah-Segal-Singer localization formula for computing $\mathcal{Q}_{K_{\beta}}\left(Y, \mathcal{E}_{d Y}, M^{\beta} \cap \Phi^{-1}(\beta), \Phi_{Y}\right)$. As we are working on a neighborhood of $Y^{\beta} \cap \Phi_{Y}^{-1}(\beta)$, we may assume that $Y$ is the normal bundle $\mathcal{N}_{Y}$ of $Y^{\beta}$ in $Y$.

Let $\mathbb{d}_{\beta}\left(\mathcal{E}_{d Y}\right)$ be the Clifford bundle on the submanifold $Y^{\beta}$ determined by $\mathcal{E}_{d Y}$ (see Section 7.1). Let $\left(\mathcal{N}_{Y}\right)_{J_{\beta}}$ be the normal bundle of $Y^{\beta}$ in $Y$ polarized by $\beta$. We apply the variation of Atiyah-Segal-Singer localisation formula to $\mathcal{N}_{Y} \rightarrow Y^{\beta}$ (the group $K$ being now $K_{\beta}$ ) and we obtain the following formula:

$$
\begin{aligned}
& \mathcal{Q}_{K_{\beta}}\left(Y, \mathcal{E}_{d Y}, M^{\beta} \cap \Phi^{-1}(\beta), \Phi_{Y}\right)= \\
& \quad \mathcal{Q}_{K_{\beta}}\left(Y^{\beta}, \mathbb{d}_{\beta}\left(\mathcal{E}_{d Y}\right) \otimes \operatorname{Sym}\left(\left(\mathcal{N}_{Y}\right)_{J_{\beta}}\right), M^{\beta} \cap \Phi^{-1}(\beta), \Phi_{Y}\right) .
\end{aligned}
$$

The manifold $Y^{\beta}$ is an open subset of $M^{\beta}$, and for the normal bundles $\mathcal{N}$ of $M^{\beta}$ in $M$ versus $\mathcal{N}_{Y}$ (the normal bundle of $Y^{\beta}$ in $Y$ ), we have $\mathcal{N}=$ $\mathcal{N}_{Y} \oplus\left[\mathfrak{k} / \mathfrak{k}_{\beta}\right]$. For the polarized ones, we get $\mathcal{N}_{J_{\beta}}=\left(\mathcal{N}_{Y}\right)_{J_{\beta}} \oplus\left[\mathfrak{q}_{J_{\beta}}\right]$. If we use the relation $\operatorname{Sym}\left(\mathfrak{q}_{J_{\beta}}\right) \otimes \wedge \mathfrak{q}_{J_{\beta}}=1$ in $\hat{R}\left(K_{\beta}\right)$, we see that

$$
\begin{aligned}
\operatorname{Sym}\left(\mathcal{N}_{J_{\beta}}\right) \otimes\left[\bigwedge\left(\mathfrak{k} / \mathfrak{k}_{\beta} \otimes \mathbb{C}\right)\right] & =\operatorname{Sym}\left(\left(\mathcal{N}_{Y}\right)_{J_{\beta}}\right) \otimes \operatorname{Sym}\left(\mathfrak{q}_{J_{\beta}}\right) \otimes\left[\bigwedge \mathfrak{q}_{J_{\beta}}\right] \otimes\left[\bigwedge \overline{\mathfrak{q}_{J_{\beta}}}\right] \\
& =\operatorname{Sym}\left(\left(\mathcal{N}_{Y}\right)_{J_{\beta}}\right) \otimes\left[\bigwedge \overline{\mathfrak{q}_{J_{\beta}}}\right] .
\end{aligned}
$$


So we are left to check that $\mathbb{d}_{\beta}\left(\mathcal{E}_{d Y}\right) \otimes\left[\wedge \mathfrak{q}_{J_{\beta}}\right]$ and $\mathbb{d}_{\beta}(\mathcal{E}) \otimes\left[\wedge \overline{\mathfrak{q} J_{\beta}}\right]$ are equal $^{5}$ as Clifford module over $\mathrm{T} Y^{\beta}$.

We have the identity $\left.\mathcal{E}\right|_{Y}=\left[\bigwedge \mathfrak{q}_{J_{\beta}}\right] \otimes \mathcal{E}_{d Y}$ as Clifford modules over $\mathfrak{k} / \mathfrak{k}_{\beta} \oplus$ $\mathrm{T} Y$. We have also $\left.\left(\mathcal{E}_{d Y}\right)\right|_{Y^{\beta}}=\bigwedge \overline{\left(\mathcal{N}_{Y}\right)_{J_{\beta}}} \otimes \mathbb{d}_{\beta}\left(\mathcal{E}_{d Y}\right)$ as an equality of Clifford modules over $\mathcal{N}_{Y} \oplus \mathrm{T} Y^{\beta}$. Finally we get

$$
\left.\mathcal{E}\right|_{Y^{\beta}}=\bigwedge \overline{\left(\mathcal{N}_{Y}\right)_{J_{\beta}}} \otimes\left[\bigwedge \mathfrak{q}_{J_{\beta}}\right] \otimes \mathbb{d}_{\beta}\left(\mathcal{E}_{d Y}\right)
$$

as an equality of Clifford modules over $\mathcal{N}_{Y} \oplus\left[\mathfrak{k} / \mathfrak{k}_{\beta}\right] \oplus \mathrm{T} Y^{\beta}$. By definition, we have also $\left.\mathcal{E}\right|_{M^{\beta}}=\bigwedge \overline{\mathcal{N}_{J_{\beta}}} \otimes \mathbb{d}_{\beta}(\mathcal{E})$ as an equality of modules over $\mathcal{N} \oplus \mathrm{T} M^{\beta}$. If we restrict the previous identity to the open subset $Y^{\beta}$, we get

$$
\left.\mathcal{E}\right|_{Y^{\beta}}=\bigwedge \overline{\left(\mathcal{N}_{Y}\right)_{J_{\beta}}} \otimes\left[\bigwedge \overline{\mathfrak{q}_{J_{\beta}}}\right] \otimes \mathbb{d}_{\beta}(\mathcal{E}) .
$$

If we use (8.28) and (8.29), we get the desired identity.

\subsection{The non abelian localization theorem}

Let $\mathcal{E} \rightarrow M$ be a equivariant graded Clifford module over an even dimensional $K$-manifold $M$ ( $M$ is not necessarily compact, the group $K$ is compact but not necessarily connected). Let $\Phi$ be a moment map, such that $Z_{\Phi}$ is compact. We can then construct $\mathcal{Q}_{K}(M, \mathcal{E}, \Phi) \in \hat{R}(K)$. If $M$ is compact, $\mathcal{Q}_{K}(M, \mathcal{E}, \Phi)$ is just equal to $\mathcal{Q}_{K}(M, \mathcal{E})$.

The set of zeroes $Z_{\Phi}$ is a disjoint union of its components $Z_{\beta}, \beta \in \mathcal{B}$, with $\mathcal{B}$ finite (see (6.16)). The following decomposition of $\mathcal{Q}_{K}(M, \mathcal{E}, \Phi)$ is the $\mathbf{K}$-theoretical analogue of Witten non abelian localization theorem in equivariant cohomology. This reduces the study of $\mathcal{Q}_{K}(M, \mathcal{E}, \Phi)$ by induction to the neighborhood of the zeroes of the moment map.

Theorem 8.6 (Non abelian localization theorem) We have

$$
\mathcal{Q}_{K}(M, \mathcal{E}, \Phi)=\sum_{\beta \in \mathcal{B}} \mathcal{Q}_{K}\left(M, \mathcal{E}, Z_{\beta}, \Phi\right)
$$

Furthermore, for $\beta \neq 0$, the generalized character $\mathcal{Q}_{K}\left(M, \mathcal{E}, Z_{\beta}, \Phi\right)$ is equal to

$$
\operatorname{Ind}_{K_{\beta}}^{K}\left(\mathcal{Q}_{K_{\beta}}\left(M^{\beta}, \mathbb{d}_{\beta}(\mathcal{E}) \otimes \operatorname{Sym}\left(\mathcal{N}_{J_{\beta}}\right), \Phi^{-1}(\beta) \cap M^{\beta}, \Phi\right) \otimes \bigwedge \mathfrak{q}_{J_{\beta}} \otimes \bigwedge \overline{\mathfrak{q}}_{J_{\beta}}\right) .
$$

\footnotetext{
${ }^{5}$ In fact we can check that $\mathbb{d}_{\beta}\left(\mathcal{E}_{d Y}\right)=(-1)^{\operatorname{dim} \mathfrak{q}_{J_{\beta}}} \operatorname{det}\left(\mathfrak{q}_{J_{\beta}}\right)^{-1} \mathbb{d}_{\beta}(\mathcal{E})$.
} 
Here, $\mathcal{B}$ is a set of representatives of the orbits of $K$ in $\Phi\left(Z_{\Phi}\right)$. For $\beta \in \mathcal{B}$, $M^{\beta}$ is the submanifold of $M$ stable by $\beta \in \mathfrak{k}, Z_{\beta}$ is $K\left(\Phi^{-1}(\beta) \cap M^{\beta}\right), \mathcal{N}_{J_{\beta}}$ is the normal bundle on $M^{\beta}$ in $M$ polarized by $\beta, \mathbb{d}_{\beta}(\mathcal{E})$ is the $K_{\beta}$ equivariant Clifford bundle on $M^{\beta}$ defined by

$$
\left.\mathcal{E}\right|_{M^{\beta}}=\mathbb{d}_{\beta}(\mathcal{E}) \otimes \bigwedge \overline{\mathcal{N}}_{J_{\beta}}
$$

and $\mathfrak{q}_{J_{\beta}}$ denotes the vector space $\mathfrak{q}=\mathfrak{k} / \mathfrak{k}_{\beta}$ equipped with the complex structure $J_{\beta}$.

As $\beta$ is a central element in $\mathfrak{k}_{\beta}$, we are reduced by induction to the study of a localized character at a central element for the lower dimensional manifold $M^{\beta}$ acted on by the subgroup $K_{\beta}$.

When 0 is a regular value of the moment map, the term $\mathcal{Q}_{K}\left(M, \mathcal{E}, \Phi^{-1}(0), \Phi\right)$ is given explicitly:

$$
\mathcal{Q}_{K}\left(M, \mathcal{E}, \Phi^{-1}(0), \Phi\right)=\sum_{\lambda \in \hat{K}} \mathcal{Q}\left(M_{\text {red }}, \mathcal{E}_{r e d} \otimes \mathcal{V}_{\lambda}^{*}\right) V_{\lambda}
$$

It follows that the multiplicities $\mathrm{m}(\lambda)$ of the irreducible representations $V_{\lambda}$ in $\mathcal{Q}_{K}\left(M, \mathcal{E}, \Phi^{-1}(0), \Phi\right)$ are given explicitly as the equivariant index of a orbi-bundle over the orbifold $M_{\text {red }}=\Phi^{-1}(0) / K$.

When $K$ is connected, we may parameterize $\hat{K}$ by the lattice of weights intersected with a positive Weyl chamber. In this case Kawasaki formula implies that $\lambda \rightarrow \mathrm{m}(\lambda)$ is a quasi-polynomial function of $\lambda$. We will return to this theme in Subsection 12.5.

\subsection{Example of $\mathrm{T}^{*} \tilde{K}$}

We consider in this subsection the paradigmatic example of the cotangent space $\mathrm{T}^{*} \tilde{K}$ where $\tilde{K}$ is a compact Lie group. This is a $\tilde{K}$-Hamiltonian manifold with moment map $\Phi$, and in the philosophy of quantization, its quantization is $L^{2}(\tilde{K})$. We here study the space $\mathcal{Q}_{\tilde{K}}\left(\mathrm{~T}^{*} \tilde{K}, \mathcal{E}, \Phi\right)$, and verify that indeed this is the space of $\tilde{K}$-finite functions on $\tilde{K}$ for a particular Clifford module $\mathcal{E}$. This example will not be used in the rest of this article.

Let $\tilde{K}$ be a compact Lie group and let $K$ be a closed subgroup. Let $i$ denote the inclusion of $K$ into $\tilde{K}, i: \mathfrak{k} \rightarrow \tilde{\mathfrak{k}}$ the induced embedding of Lie algebras, and $\pi: \tilde{\mathfrak{k}}^{*} \rightarrow \mathfrak{k}^{*}$ the dual projection.

We consider the following action of $\tilde{K} \times K$ on $\tilde{K}:(\tilde{k}, k) \cdot a=\tilde{k} a k^{-1}$.

The tangent bundle T $\tilde{K}$ is identified with $\tilde{K} \times \tilde{\mathfrak{k}}$ through the left translations: to $(a, X) \in \tilde{K} \times \tilde{\mathfrak{k}}$ we associate $\left.\frac{d}{d t} e^{t X} a\right|_{t=0} \in \mathrm{T}_{a} \tilde{K}$. The action of 
$\tilde{K} \times K$ on the cotangent bundle $\mathrm{T}^{*} \tilde{K} \simeq \tilde{K} \times \tilde{\mathfrak{k}}^{*}$ is then

$$
(\tilde{k}, k) \cdot(a, \xi)=\left(\tilde{k} a k^{-1}, \tilde{k} \xi\right) .
$$

The Liouville form $\lambda$ of $\mathrm{T}^{*} \tilde{K}$ is thus given by $\lambda_{a, \xi}(X, \eta)=\langle\xi, X\rangle$ for $(a, \xi) \in \mathrm{T}^{*} \tilde{K} \simeq \tilde{K} \times \tilde{\mathfrak{k}}$ and $(X, \eta) \in \mathrm{T}_{a} \tilde{K} \times \mathrm{T}_{\xi} \tilde{\mathfrak{k}}$. The symplectic form on $\mathrm{T}^{*} \tilde{K}$ is $\Omega:=d \lambda$.

The corresponding moment map relative to the $\tilde{K} \times K$-action is the map $\Phi=\Phi_{\tilde{K}} \oplus \Phi_{K}: \mathrm{T}^{*} \tilde{K} \rightarrow \tilde{\mathfrak{k}}^{*} \oplus \mathfrak{k}^{*}$ defined by

$$
\Phi_{\tilde{K}}(a, \xi)=-\xi, \quad \Phi_{K}(a, \xi)=\pi\left(a^{-1} \cdot \xi\right) .
$$

Lemma 8.7 The set $Z_{\Phi} \subset \mathrm{T}^{*} \tilde{K}$ is equal to $\tilde{K} \times\{0\}$. More generally, if we consider the equivariant map $\Phi_{t}=\Phi_{\tilde{K}} \oplus t \Phi_{K}$ for any $t \geq 0$, the set $Z_{\Phi_{t}} \subset \mathrm{T}^{*} \tilde{K}$ is still equal to $\tilde{K} \times\{0\}$.

Proof. Let $\kappa_{t}$ be the Kirwan vector field on $\mathrm{T}^{*} \tilde{K}$ attached to the map $\Phi_{t}$. A direct computation gives that for $(a, \xi) \in \mathrm{T}^{*} \tilde{K}$, the vector $\kappa_{t}(a, \xi) \in \mathrm{T}_{(a, \xi)}\left(\tilde{K} \times \tilde{\mathfrak{k}}^{*}\right)=\tilde{\mathfrak{k}} \times \tilde{\mathfrak{k}}^{*}$ is equal to $\left(\kappa_{t}^{1}(a, \xi), 0\right)$ with

$$
\kappa_{t}^{1}(a, \xi)=\xi+\operatorname{ta} \pi\left(a^{-1} \xi\right) .
$$

For $t \geq 0$, we see that $\kappa_{t}^{1}(a, \xi)=0$ if and only if $\xi=0$.

Let $\mathcal{E}$ be a $\tilde{K} \times K$-equivariant graded Clifford bundle on the manifold $\mathrm{T}^{*} \tilde{K}$. Thanks to Lemma 8.7 , we know that $Z_{\Phi}$ is compact. Hence we can consider the generalized index

$$
\mathcal{Q}_{\tilde{K} \times K}\left(\mathrm{~T}^{*} \tilde{K}, \mathcal{E}, \Phi\right) \in \hat{R}(\tilde{K} \times K) .
$$

The restriction of $\mathcal{E}$ at $(1,0) \in \mathrm{T}^{*} \tilde{K}$ defines a graded Clifford module $\left.\mathcal{E}\right|_{(1,0)}$ for the Euclidean vector space $\left.\mathrm{T}\left(\mathrm{T}^{*} \tilde{K}\right)\right|_{(1,0)} \simeq \tilde{\mathfrak{k}} \times \tilde{\mathfrak{k}}^{*}$. We use the identification $\tilde{\mathfrak{k}} \times \tilde{\mathfrak{k}}^{*} \simeq \tilde{\mathfrak{k}}_{\mathbb{C}},(X, \xi) \mapsto X \oplus i \xi$. Thus $\bigwedge \tilde{\mathfrak{k}}_{\mathbb{C}}$ is an irreducible Clifford module for $\tilde{\mathfrak{k}} \times \tilde{\mathfrak{k}}^{*}$.

Following Definition 7.1, we can "divide" the graded Clifford bundle $\left.\mathcal{E}\right|_{(1,0)}$ by the irreducible Clifford module $\wedge \mathfrak{k}_{\mathbb{C}}$.

Definition 8.8 Let $E_{\tilde{K} \times K}$ be the graded $\tilde{K} \times K$-representation space such that

$$
\left.\mathcal{E}\right|_{(1,0)} \simeq E_{\tilde{K} \times K} \otimes \bigwedge \mathfrak{k}_{\mathbb{C}} .
$$

is an isomorphism of graded Clifford modules on $\tilde{\mathfrak{k}} \times \tilde{\mathfrak{k}}^{*}$. 
Proposition 8.9 We have the following relation in $\hat{R}(\tilde{K} \times K)$ :

$$
\mathcal{Q}_{\tilde{K} \times K}\left(\mathrm{~T}^{*} \tilde{K}, \mathcal{E}, \Phi\right)=\sum_{V \in \widehat{\tilde{K}}}\left(\left.V^{*} \otimes V\right|_{K}\right) \otimes E_{\tilde{K} \times K} .
$$

Remark 8.10 If $E_{\tilde{K} \times K}=\mathbb{C}$, we obtain that $\mathcal{Q}_{\tilde{K} \times K}\left(\mathrm{~T}^{*} \tilde{K}, \mathcal{E}, \Phi\right)$ is $L^{2}(\tilde{K})$ after completion, as expected.

Proof. Thanks to Lemma 8.7, we know that $t \in[0,1] \mapsto \sigma\left(\mathrm{T}^{*} \tilde{K}, \mathcal{E}, \Phi_{t}\right)$ is an homotopy of transversally elliptic symbols. It follows that $\mathcal{Q}_{\tilde{K} \times K}\left(\mathrm{~T}^{*} \tilde{K}, \mathcal{E}, \Phi\right)=\mathcal{Q}_{\tilde{K} \times K}\left(\mathrm{~T}^{*} \tilde{K}, \mathcal{E}, \Phi_{\tilde{K}}\right)$. We note that the computation of $\mathcal{Q}_{\tilde{K} \times K}\left(\mathrm{~T}^{*} K, \mathcal{E}, \Phi_{\tilde{K}}\right)$ follows from (8.26), since $Z_{\Phi_{\tilde{K}}}=\Phi_{\tilde{K}}^{-1}(0), 0$ is a regular value of $\Phi_{\tilde{K}}$ and $\Phi_{\tilde{K}}^{-1}(0) / \tilde{K}$ is reduced to a point.

\section{$9[Q, R]=0$ Theorem}

Let $M$ be an even dimensional $K$-manifold equipped with an equivariant graded Clifford bundle $\mathcal{E}$.

When $M$ is compact, we consider the representation $\mathcal{Q}_{K}(M, \mathcal{E})$. In this section, we study $\left[\mathcal{Q}_{K}(M, \mathcal{E})\right]^{K}$, with the help of a moment map $\Phi$, and the non abelian localization formula, in particular, we will find sufficient conditions on the couple $(\mathcal{E}, \Phi)$ in order that the only component of $Z_{\Phi}$ contributing to $\left[\mathcal{Q}_{K}(M, \mathcal{E})\right]^{K}$ is $\Phi^{-1}(0)$. We will be considering in more detail the case where $M$ is an almost complex manifold.

Our discussion extends to the case where $M$ is non-compact. We will study the generalized character $\mathcal{Q}_{K}(M, \mathcal{E}, \Phi)$ attached to a moment map $\Phi$ such that $Z_{\Phi}$ is compact.

\subsection{A preliminary formula}

Let $M$ be an even dimensional $K$-manifold equipped with an equivariant graded Clifford bundle $\mathcal{E}$ and a moment map $\Phi: M \rightarrow \mathfrak{k}^{*}$ such that $Z_{\Phi}$ is compact. We study $\left[\mathcal{Q}_{K}(M, \mathcal{E}, \Phi)\right]^{K}$ via the non abelian localization formula. The notations in this subsection are as in Subsection 8.4. From Theorem 8.6, we obtain

$$
\left[\mathcal{Q}_{K}(M, \mathcal{E}, \Phi)\right]^{K}=\sum_{\beta \in \mathcal{B}}\left[\mathcal{Q}_{K}\left(M, \mathcal{E}, Z_{\beta}, \Phi\right)\right]^{K}
$$

and if $\beta \neq 0$, the integer $\left[\mathcal{Q}_{K}\left(M, \mathcal{E}, Z_{\beta}, \Phi\right)\right]^{K}$ is equal to

$$
\left[\mathcal{Q}_{K_{\beta}}\left(M^{\beta}, \mathbb{d}_{\beta}(\mathcal{E}) \otimes \operatorname{Sym}\left(\mathcal{N}_{J_{\beta}}\right), \Phi^{-1}(\beta) \cap M^{\beta}, \Phi\right) \otimes \bigwedge \mathfrak{q}_{J_{\beta}} \otimes \bigwedge \overline{\mathfrak{q}}_{J_{\beta}}\right]^{K_{\beta}} .
$$


Using Lemma 4.1, the vanishing of this last expression is automatic if the linear action (denoted $\mathcal{L}(\beta)$ ) of $\beta$ on the fibers of $\mathbb{d}_{\beta}(\mathcal{E}) \otimes \operatorname{Sym}\left(\mathcal{N}_{J_{\beta}}\right) \otimes \wedge \mathfrak{q}_{J_{\beta}} \otimes$ $\wedge \overline{\mathfrak{q}}_{J_{\beta}}$ has only non-zero eigenvalues. We thus introduce some definitions.

Consider a $K_{\beta}$-equivariant vector bundle $\mathcal{F} \rightarrow M^{\beta}$. Let $\mathcal{X}$ be a connected component of $M^{\beta}$. Let $c \in \mathbb{R}$. If all the eigenvalues of $\mathcal{L}(\beta)$ acting on $\left.\mathcal{F}\right|_{\mathcal{X}}$ are of the form $i \theta$ with $\theta>c$ (respectively $\theta \geq c$ ), we say that $\frac{1}{i} \mathcal{L}(\beta)>c$ on $\left.\mathcal{F}\right|_{\mathcal{X}}\left(\right.$ respectively $\left.\frac{1}{i} \mathcal{L}(\beta) \geq c\right)$.

Proposition 9.1 Let $\beta \neq 0$.

- If $\frac{1}{i} \mathcal{L}(\beta) \geq 0$ on $\left.\mathbb{d}_{\beta}(\mathcal{E})\right|_{\mathcal{X}} \otimes \operatorname{det}\left(\mathfrak{q}_{J_{\beta}}\right)^{-1}$ for any connected component $\mathcal{X} \subset M^{\beta}$ such that $\mathcal{X} \cap \Phi^{-1}(\beta) \neq \emptyset$. Then

$$
\left[\mathcal{Q}_{K}\left(M, \mathcal{E}, Z_{\beta}, \Phi\right)\right]^{K}=\left[\mathcal{Q}_{K_{\beta}}\left(M^{\beta}, \mathcal{E}_{[\beta]}, \Phi^{-1}(\beta) \cap M^{\beta}, \Phi\right)\right]^{K_{\beta}}
$$

where

$$
\mathcal{E}_{[\beta]}:=(-1)^{\operatorname{dim} \mathfrak{q}_{J_{\beta}}}\left[\mathbb{d}_{\beta}(\mathcal{E}) \otimes \operatorname{det}\left(\mathfrak{q}_{J_{\beta}}\right)^{-1}\right]^{\beta}
$$

is a graded $K_{\beta}$-equivariant Clifford bundle on $M^{\beta}$.

- If $\frac{1}{i} \mathcal{L}(\beta)>0$ on $\left.\mathbb{d}_{\beta}(\mathcal{E})\right|_{\mathcal{X}} \otimes \operatorname{det}\left(\mathfrak{q}_{J_{\beta}}\right)^{-1}$ for any connected component $\mathcal{X} \subset M^{\beta}$ such that $\mathcal{X} \cap \Phi^{-1}(\beta) \neq \emptyset$, we have $\mathcal{E}_{[\beta]}=[0]$ in a neighborhood of $M^{\beta} \cap \Phi^{-1}(\beta)$ and then $\left[\mathcal{Q}_{K}\left(M, \mathcal{E}, Z_{\beta}, \Phi\right)\right]^{K}=0$.

Remark that $\left[\mathbb{d}_{\beta}(\mathcal{E}) \otimes \operatorname{det}\left(\mathfrak{q}_{J_{\beta}}\right)^{-1}\right]^{\beta}$ is isomorphic to the subbundle of $\mathbb{d}_{\beta}(\mathcal{E})$ formed by the vectors satisfying $\mathcal{L}(\beta) v=\operatorname{Tr}_{\mathfrak{q}_{J_{\beta}}}(\beta) v$.

\section{Proof.}

Introduce the union $M_{c}^{\beta}=\cup \mathcal{X}$ of the connected components $\mathcal{X}$ of $M^{\beta}$ such that $\mathcal{X}$ intersects $\Phi^{-1}(\beta)$. Then $M_{c}^{\beta}$ is $K_{\beta}$ invariant and clearly, we can replace $M^{\beta}$ by $M_{c}^{\beta}$ in Theorem 8.6. Using Lemma 4.1, we obtain $\left[\mathcal{Q}_{K}\left(M, \mathcal{E}, Z_{\beta}, \Phi\right)\right]^{K}$ is equal to

$$
\left[\mathcal{Q}_{K_{\beta}}\left(M_{c}^{\beta},\left[\mathcal{F}_{M_{c}^{\beta}}\right]^{\beta}, M_{c}^{\beta} \cap \Phi^{-1}(\beta), \Phi\right)\right]^{K_{\beta}}
$$

where $\mathcal{F}_{M_{c}^{\beta}}=\left.\left.\mathbb{d}_{\beta}(\mathcal{E})\right|_{M_{c}^{\beta}} \otimes\left(\operatorname{det}\left(\mathfrak{q}_{J_{\beta}}\right)^{-1} \otimes \operatorname{det}\left(\mathfrak{q}_{J_{\beta}}\right)\right) \otimes \operatorname{Sym}\left(\mathcal{N}_{J_{\beta}}\right)\right|_{M_{c}^{\beta}} \otimes \bigwedge \mathfrak{q}_{J_{\beta}} \otimes$ $\bigwedge \overline{\mathfrak{q} J_{\beta}}$.

Let $\mathcal{X}$ be a connected component of $M_{c}^{\beta}$, thus $\mathcal{X}$ intersects $\Phi^{-1}(\beta)$. On $\left.\operatorname{Sym}\left(\mathcal{N}_{J_{\beta}}\right)\right|_{\mathcal{X}} \otimes \wedge \mathfrak{q}_{J_{\beta}}$, we have $\frac{1}{i} \mathcal{L}(\beta) \geq 0$ by definition of the complex structures associated to $\beta$, and $\left[\left.\operatorname{Sym}\left(\mathcal{N}_{J_{\beta}}\right)\right|_{\mathcal{X}} \otimes \wedge \mathfrak{q}_{J_{\beta}}\right]^{\beta}=[\mathbb{C}]$. Similarly 
$\frac{1}{i} \mathcal{L}(\beta) \geq 0$ on $\left[\operatorname{det}\left(\mathfrak{q}_{J_{\beta}}\right) \otimes \wedge \overline{\mathfrak{q}_{J_{\beta}}}\right]$ and $\left[\operatorname{det}\left(\mathfrak{q}_{J_{\beta}}\right) \otimes \wedge \overline{\mathfrak{q}_{J_{\beta}}}\right]^{\beta}=(-1)^{\operatorname{dim} \mathfrak{q}_{J_{\beta}}}$ Then the Clifford bundle $\left[\mathcal{F}_{\mathcal{X}}\right]^{\beta}$ is reduced to $\mathcal{E}_{[\beta]}$ if $\frac{1}{i} \mathcal{L}(\beta) \geq 0$ on $\left.\mathbb{d}_{\beta}(\mathcal{E})\right|_{\mathcal{X}} \otimes$ $\operatorname{det}\left(\mathfrak{q}_{J_{\beta}}\right)^{-1}$. This proves the first point. The second point is an obvious consequence of the first.

\section{$9.2 \Phi$-positivity}

Definition 9.2 Let $\Phi: M \rightarrow \mathfrak{k}^{*}$ be an equivariant map.

- A K-equivariant bundle $\mathcal{F} \rightarrow M$ is weakly $\Phi$-positive (resp. strictly $\Phi$-positive) if

$$
\frac{1}{i} \mathcal{L}(\beta) \geq 0 \quad(\text { resp. }>0) \quad \text { on }\left.\quad \mathcal{F}\right|_{\mathcal{X}}
$$

for any couple $(\mathcal{X}, \beta)$ where $\mathcal{X} \subset M^{\beta}$ is a connected component such that $\mathcal{X} \cap \Phi^{-1}(\beta) \neq \emptyset$, and $\beta \neq 0$.

- A K-equivariant Clifford bundle $\mathcal{E} \rightarrow M$ is weakly $\Phi$-positive (resp. strictly $\Phi$-positive) if

$$
\frac{1}{i} \mathcal{L}(\beta) \geq 0 \quad(\text { resp. }>0) \quad \text { on }\left.\quad \mathbb{d}_{\beta}(\mathcal{E})\right|_{\mathcal{X}} \otimes \operatorname{det}\left(\mathfrak{q}_{J_{\beta}}\right)^{-1}
$$

for any couple $(\mathcal{X}, \beta)$ where $\mathcal{X} \subset M^{\beta}$ is a connected component such that $\mathcal{X} \cap \Phi^{-1}(\beta) \neq \emptyset$ and $\beta \neq 0$.

An obvious example of weakly $\Phi$-positive bundle $\mathcal{F}$ on $M$ is the trivial bundle $M \times \mathbb{C}$.

If $\mathcal{E}$ is a weakly $\Phi$-positive Clifford bundle and $\mathcal{F}$ a weakly $\Phi$-positive bundle, then the Clifford module $\mathcal{E} \otimes \mathcal{F}$ is weakly $\Phi$-positive.

Another example arise when we have an equivariant map $\varphi: M^{\prime} \rightarrow M$. Suppose that we start with a weakly $\Phi$-positive bundle $\mathcal{F}$ on $M$. We consider the pull-backs $\mathcal{F}^{\prime}=\varphi^{*} \mathcal{F}$ and $\Phi^{\prime}=\varphi^{*} \Phi$ on $M^{\prime}$. It is obvious that $\varphi\left(\left\{\kappa_{\Phi^{\prime}}=\right.\right.$ $0\}) \subset\left\{\kappa_{\Phi}=0\right\}$. It is a small exercise to check the following lemma.

Lemma 9.3 If $\varphi\left(\left\{\kappa_{\Phi^{\prime}}=0\right\}\right)=\left\{\kappa_{\Phi}=0\right\}$, the bundle $\mathcal{F}^{\prime}$ is weakly $\Phi^{\prime}$ positive

A particularly important example of strictly $\Phi$-positive bundle is the following.

Definition 9.4 A $K$-equivariant line bundle $L$ over $M$ is called a $\Phi$-moment line bundle if, for any $m \in M$ and any $X \in \mathfrak{k}_{m}$, the action of $\mathcal{L}(X)$ on the fiber $\left.L\right|_{m}$ is equal to $i\langle\Phi(m), X\rangle \mathrm{Id}$. 
Such a line bundle $L$ is strictly $\Phi$-positive, since for any $m \in \Phi^{-1}(\beta) \cap$ $M^{\beta}$, the action of $\mathcal{L}(\beta)$ on $\left.L\right|_{m}$ is equal to $i\|\beta\|^{2} \mathrm{Id}$. In particular, let $\Phi_{L}$ be the moment map associated to an Hermitian connection (Definition 6.3). Clearly $L$ is a $\Phi_{L}$-line bundle, and the line bundle $L$ is strictly $\Phi_{L}$-positive.

Example 9.5 Let us consider the case studied in Section 8.5: the Hamiltonian action of $\tilde{K} \times K$ on $\mathrm{T}^{*} \tilde{K}$. The moment map is $\Phi(a, \xi)=-\xi \oplus \pi\left(a^{-1} \xi\right)$. In this situation, the trivial line bundle over $\mathrm{T}^{*} \tilde{K}$ is a $\Phi$-moment line bundle.

The following theorem follows from Proposition 9.1.

Theorem 9.6 Let $M$ be a $K$-manifold equipped with a moment map $\Phi$ such that $Z_{\Phi}$ is compact. Let $\mathcal{E}$ be an equivariant graded Clifford bundle on $M$.

- If $\mathcal{E}$ is weakly $\Phi$-positive, then we have

$$
\begin{aligned}
& {\left[\mathcal{Q}_{K}(M, \mathcal{E}, \Phi)\right]^{K}=\left[\mathcal{Q}_{K}\left(M, \mathcal{E}, \Phi^{-1}(0), \Phi\right)\right]^{K}} \\
& \quad+\sum_{\beta \in \mathcal{B}, \beta \neq 0}\left[\mathcal{Q}_{K_{\beta}}\left(M^{\beta}, \mathcal{E}_{[\beta]}, M^{\beta} \cap \Phi^{-1}(\beta), \Phi\right)\right]^{K_{\beta}}
\end{aligned}
$$

where the Clifford bundles $\mathcal{E}_{[\beta]}$ are defined by (9.31).

- When the Clifford bundle $\mathcal{E}$ is strictly $\Phi$-positive, we have

$$
\left[\mathcal{Q}_{K}(M, \mathcal{E}, \Phi)\right]^{K}=\left[\mathcal{Q}_{K}\left(M, \mathcal{E}, \Phi^{-1}(0), \Phi\right)\right]^{K} .
$$

If furthermore 0 is a regular value of $\Phi$, then $K$ acts locally freely on $\Phi^{-1}(0)$, and we have $\left[\mathcal{Q}_{K}(M, \mathcal{E})\right]^{K}=\mathcal{Q}\left(M_{\text {red }}, \mathcal{E}_{\text {red }}\right)$.

The equality $\left[\mathcal{Q}_{K}\left(M, \mathcal{E}, \Phi^{-1}(0), \Phi\right)\right]^{K}=\mathcal{Q}\left(M_{\text {red }}, \mathcal{E}_{\text {red }}\right)$ is proved in Theorem 8.3.

Finally let us remark the following.

Proposition 9.7 Let $\Phi$ be a moment map, and assume that $L$ is a $\Phi$ moment line bundle. Let $\Phi_{t}, t \in[0,1]$ be a smooth family of moment maps such that $\Phi_{0}=\Phi$. We assume that there exists a relatively compact open subset $\mathcal{U} \subset M$ such that $Z_{\Phi_{t}} \subset \mathcal{U}$ for all $t \in[0,1]$. Then there exists $\epsilon>0$ such that $L$ is weakly $\Phi_{t}$-positive for any $t \in[0, \epsilon]$.

Proof. Take the notations of Lemma 6.7. Consider a finite set $\left\{\mathfrak{k}_{i}\right\}$ of representatives of the infinitesimal stabilizers of the action of $K$ on $\mathcal{U}$, and let $\mathcal{C}_{i}$ be the set of connected components $\mathcal{X}$ of $M_{\mathfrak{k}_{i}} \cap \mathcal{U}$. The action of $\frac{1}{i} \beta(\mathcal{X}, \Phi) \in \mathfrak{k}_{i}$ on $\left.L\right|_{m}$, for any $m \in \mathcal{X}$, is given by $\|\beta(\mathcal{X}, \Phi)\|^{2} \operatorname{Id}$ as $\beta(\mathcal{X}, \Phi) \in \mathfrak{k}_{i}$ and $\Phi(\mathcal{X}) \subset \beta(\mathcal{X}, \Phi)+\mathfrak{k}_{i}^{\perp}$. 
Let $m \in Z_{\Phi_{t}} \subset \mathcal{U}$. Then, up to conjugacy, there exists an $i$ such that $m \in \Phi_{t}^{-1}\left(\mathfrak{k}_{i}\right) \cap M_{\mathfrak{k}_{i}} \cap \mathcal{U}$. Let $\mathcal{X}$ be the connected component of $M_{\mathfrak{k}_{i}} \cap \mathcal{U}$ containing $m$. Then $\beta_{t}=\Phi_{t}(m)=\beta\left(\mathcal{X}, \Phi_{t}\right) \in \mathfrak{k}_{i}$ and is close to the point $\beta(\mathcal{X}, \Phi) \in \mathfrak{k}_{i}$. Thus if $\beta(\mathcal{X}, \Phi) \neq 0$, and $t$ small enough, the action of $\beta_{t}$ is positive on $\left.L\right|_{m}$. If $\beta(\mathcal{X}, \Phi)=0$, then $\Phi(\mathcal{X})$ is contained in $\mathfrak{k}_{i}^{\perp}$, and so the action of $\beta_{t} \in \mathfrak{k}_{i}$ on $\left.L\right|_{m}$ is equal to $i\left\langle\Phi(m), \beta_{t}\right\rangle=0$.

\section{3 $[Q, R]=0$ in the asymptotic sense}

Let $M$ be a compact even dimensional $K$-manifold equipped with an equivariant graded Clifford bundle $\mathcal{E}$. Let $L$ be a $K$-equivariant Hermitian line bundle with Hermitian connection $\nabla$, and consider its moment map $\Phi_{L}$.

Theorem 9.8 When $k$ is sufficiently large, we have

$$
\left[\mathcal{Q}_{K}\left(M, \mathcal{E} \otimes L^{\otimes k}\right)\right]^{K}=\left[\mathcal{Q}_{K}\left(M, \mathcal{E} \otimes L^{\otimes k}, \Phi_{L}^{-1}(0), \Phi_{L}\right)\right]^{K} .
$$

If furthermore 0 is a regular value of $\Phi_{L}$, then $K$ acts locally freely on $\Phi_{L}^{-1}(0)$, and we have $\left[\mathcal{Q}_{K}\left(M, \mathcal{E} \otimes L^{\otimes k}\right)\right]^{K}=\mathcal{Q}\left(M_{\text {red }}, \mathcal{E}_{\text {red }} \otimes L_{\text {red }}^{\otimes k}\right)$.

This theorem follows right away from Theorem 9.6. Indeed, when $\beta \neq 0$, for any couple $(\mathcal{X}, \beta)$ where $\mathcal{X} \subset M^{\beta}$ is a connected component such that $\mathcal{X} \cap \Phi_{L}^{-1}(\beta) \neq \emptyset$, the action of $\beta / i$ on $\left.L^{\otimes k}\right|_{\mathcal{X}}$ is given by $k\|\beta\|^{2}$, so when $k$ is large, the Clifford bundle $\mathcal{E} \otimes L^{\otimes k}$ is strictly $\Phi_{L}$-positive.

So, although the map $\Phi_{L}$ varies with the choice of connection on $L$ and the topological space $M_{\text {red }}$ may vary dramatically, then for $k$ large, the quantity $\mathcal{Q}\left(M_{\text {red }}, \mathcal{E}_{\text {red }} \otimes L_{r e d}^{\otimes k}\right)$ is independent of the choice of the connection on $L$.

Theorem 9.32 plays an essential role when one studies the asymptotic behaviour of branching law coefficients (see [37]).

\section{$10[Q, R]=0$ for almost complex manifolds}

Let us assume that our $K$-manifold $M$ is provided with a $K$-invariant almost complex structure $J$. Then, as in the complex case (see Example 5.11), a natural Clifford bundle on $M$ is $\bigwedge_{J} \mathrm{~T} M$ and any Clifford module on $M$ is a twisted Clifford bundle $\bigwedge_{J} \mathrm{~T} M \otimes \mathcal{F}$ where $\mathcal{F}$ is a $K$-equivariant complex vector bundle. 
Definition 10.1 • When $M$ is compact, we define the Riemann-Roch character

$$
R R_{K}^{J}(M, \mathcal{F}):=\mathcal{Q}_{K}\left(M, \bigwedge_{J} \mathrm{~T} M \otimes \mathcal{F}\right) .
$$

- When $M$ is not necessarily compact, we can attach the localized RiemannRoch character

$$
R R_{K}^{J}(M, \mathcal{F}, \Phi):=\mathcal{Q}_{K}\left(M, \bigwedge_{J} \mathrm{~T} M \otimes \mathcal{F}, \Phi\right)
$$

to any equivariant map $\Phi: M \rightarrow \mathfrak{k}^{*}$ such that $Z_{\Phi}$ is compact.

- When $Z$ is a compact component of $Z_{\Phi}$, we define the localized RiemannRoch character by

$$
R R_{K}^{J}(M, \mathcal{F}, Z, \Phi):=\mathcal{Q}_{K}\left(M, \bigwedge_{J} \mathrm{~T} M \otimes \mathcal{F}, Z, \Phi\right) .
$$

An important example of almost complex manifold is the case of symplectic manifold $(M, \Omega)$. In this case, we construct $J$ as follows: choose a $K$-invariant Riemannian metric $g$ on $M$, and write $\Omega_{m}(v, w)=g\left(v, A_{m} w\right)$ where $A_{m}$ is a invertible antisymmetric matrix, and choose $J_{m}=\frac{A_{m}}{\sqrt{-A_{m} A_{m}^{*}}}$. $\Phi$.

Our aim is to compute $\left[R R_{K}^{J}(M, \mathcal{F}, \Phi)\right]^{K}$ for a well chosen moment map

\subsection{Statement of the results}

We start with a definition.

Definition 10.2 Consider the data $(\Omega, J)$ where $\Omega$ is a two form and $J$ is an almost complex structure on $M$. We say that $(\Omega, J)$ is adapted if, for any $m \in M, \Omega(J v, J w)=\Omega(v, w)$ and the quadratic form $v \in \mathrm{T}_{m} M \mapsto \Omega(v, J v)$ is semi-positive.

We have the fundamental fact that we will prove in next subsection.

Proposition 10.3 If $(\Omega, \Phi)$ is a weakly Hamiltonian structure on $M$ such that $(\Omega, J)$ is adapted, then the Clifford bundle $\bigwedge_{J} \mathrm{~T} M$ is weakly $\Phi$-positive.

For the rest of this section, we work with a weakly Hamiltonian structure $(\Omega, \Phi)$ on $M$ such that $(\Omega, J)$ is adapted.

Consider $\beta \in \mathfrak{k}$. For the tangent bundle, we have the decomposition $\left.\mathrm{T} M\right|_{M^{\beta}}=\mathrm{T} M^{\beta} \oplus \mathcal{N}$, where $\mathrm{T}^{\beta}=\operatorname{ker}(\mathcal{L}(\beta))$ and $\mathcal{N}=\operatorname{Image}(\mathcal{L}(\beta))$. 
Since the complex structure $J$ on $M$ commutes with $\mathcal{L}(\beta)$, the vector subbundles $\mathrm{T}^{\beta}$ and $\mathcal{N}$ are stable under $J$. On $\mathcal{N}$, as we have another complex structure $J_{\beta}$ that commutes with $J$, we get the decomposition $\mathcal{N}=\mathcal{N}_{J_{\beta},+} \oplus$ $\mathcal{N}_{J_{\beta},-}$ where $\mathcal{N}_{J_{\beta}, \pm}=\left\{v \in \mathcal{N} \mid J_{\beta} v= \pm J v\right\}$.

Also this lemma will be proved in the next subsection.

Lemma 10.4 For any non-zero element $\beta \in \mathfrak{k}$, and any $m \in M^{\beta} \cap \Phi^{-1}(\beta)$, the real dimension of $\left.\mathcal{N}_{J_{\beta},+}\right|_{m}$ is greater or equal than $\operatorname{dim}_{\mathbb{R}}\left(\mathfrak{k} / \mathfrak{k}_{\beta}\right)$.

Recall that the critical set $Z_{\Phi}$ is parameterized by a finite set $\mathcal{B}$. Let us fix a non-zero element $\beta \in \mathcal{B}$.

Definition 10.5 For any non-zero element $\beta \in \mathcal{B}$, define $M_{o}^{\beta} \subset M^{\beta}$ as the union $\cup \mathcal{X}$ of the connected components $\mathcal{X}$ of $M^{\beta}$ intersecting $\Phi^{-1}(\beta)$ and such that the real rank of the vector bundle $\mathcal{N}_{J_{\beta},+} \mid \mathcal{X}$ is equal to $\operatorname{dim}_{\mathbb{R}}\left(\mathfrak{k} / \mathfrak{k}_{\beta}\right)$.

We notice that $M_{o}^{\beta}$ is a submanifold of $M^{\beta}$ stable under the action of the group $K_{\beta}$.

The main result of this subsection is the following theorem.

Theorem 10.6 Let $\mathcal{F}$ be a $K$-equivariant bundle on an almost complex manifold $(M, J)$. Let $(\Omega, \Phi)$ be a weakly Hamiltonian structure such that $(\Omega, J)$ is adapted and $Z_{\Phi}$ is compact.

- If the vector bundle $\mathcal{F}$ is weakly $\Phi$-positive, we have

$$
\begin{aligned}
& {\left[R R_{K}^{J}(M, \mathcal{F}, \Phi)\right]^{K}=\left[R R_{K}\left(M, \mathcal{F}, \Phi^{-1}(0), \Phi\right)\right]^{K}+} \\
& \quad \sum_{\beta \in \mathcal{B} \backslash\{0\}}\left[R R_{K_{\beta}}\left(M_{o}^{\beta},\left[\left.\mathcal{F}\right|_{M_{o}^{\beta}}\right]^{\beta}, M_{o}^{\beta} \cap \Phi^{-1}(\beta), \Phi\right)\right]^{K_{\beta}} .
\end{aligned}
$$

- If the vector bundle $\mathcal{F}$ is strictly $\Phi$-positive, then

$$
\left[R R_{K}^{J}(M, \mathcal{F}, \Phi)\right]^{K}=\left[R R_{K}\left(M, \mathcal{F}, \Phi^{-1}(0), \Phi\right)\right]^{K} .
$$

- If 0 is a regular value of $\Phi$, we have

$$
\left[R R_{K}\left(M, \mathcal{F}, \Phi^{-1}(0), \Phi\right)\right]^{K}=\mathcal{Q}\left(M_{\text {red }}, \mathcal{S}_{\text {red }} \otimes \mathcal{F}_{\text {red }}\right) .
$$

Here $\mathcal{F}_{\text {red }}=\left.\mathcal{F}\right|_{\Phi^{-1}(0)} / K$ is a orbibundle over $M_{\text {red }}=\Phi^{-1}(0) / K$, and $\mathcal{S}_{\text {red }}$ is the Spin ${ }^{c}$-bundle on $M_{\text {red }}$ induced by $\bigwedge_{J} \mathrm{~T} M$. 
Suppose now that $(\Omega, \Phi)$ is an Hamiltonian structure : the closed 2-form $\Omega$ is non-degenerate. When $M$ is non-compact, we assume furthermore that $\Phi$ is proper and that the set $Z_{\Phi}$ is compact. Theorem 10.6 can be improved as follows.

Theorem 10.7 Let $(M, J)$ be an almost complex manifold, and let $\mathcal{F}$ be a $K$-equivariant vector bundle. Let $(\Omega, \Phi)$ be a Hamiltonian structure on $M$ such that : $(\Omega, J)$ is adapted, the moment map $\Phi$ is proper and $Z_{\Phi}$ is compact.

If the vector bundle $\mathcal{F}$ is weakly $\Phi$-positive, and 0 is in the image of the moment map, then:

$$
\left[R R_{K}^{J}(M, \mathcal{F}, \Phi)\right]^{K}=\left[R R_{K}^{J}\left(M, \mathcal{F}, \Phi^{-1}(0), \Phi\right)\right]^{K} .
$$

Our manifold $M$ is not necessarily connected, but, considering the action of $K$ on connected components of $M$, our study reduces easily to the case where $M=K M^{0}$, where $M^{0}$ is connected. Then $M^{0}$ is connected and stable by the connected component $K^{0}$ of $K$. We now assume that we are in this situation. In the Hamiltonian setting, we then have the following lemma.

Lemma 10.8 The set of points in $\Phi(M)$ at minimum distance of the origin is a $K$-orbit $K \beta_{\min }$. Furthermore $\Phi^{-1}\left(K \beta_{\min }\right) \subset M^{\beta_{\min }}$.

We denote by $M^{\prime}=M^{\beta_{\min }}, K^{\prime}=K_{\beta_{\min }}, \Phi^{\prime}=\left.\Phi\right|_{M^{\prime}}-\beta_{\min }, J^{\prime}$ the restriction of $J$ to $M^{\prime}$, and $\mathcal{F}^{\prime}$ the restriction of $\mathcal{F}$ to $M^{\prime}$. We have then the following theorem, which allows us to always reduce the study of $\left[R R_{K}^{J}(M, \mathcal{F}, \Phi)\right]^{K}$ when $\mathcal{F}$ is weakly $\Phi$-positive, to the study of a Hamiltonian manifold with 0 in the image of its moment map.

Theorem 10.9 Let $(M, J)$ be an almost complex manifold, and let $\mathcal{F}$ be a $K$-equivariant vector bundle. Let $(\Omega, \Phi)$ be a Hamiltonian structure on $M$ such that : $(\Omega, J)$ is adapted, the moment map $\Phi$ is proper and $Z_{\Phi}$ is compact. If the vector bundle $\mathcal{F}$ is weakly $\Phi$-positive, then $\mathcal{F}^{\prime}$ is weakly $\Phi^{\prime}$-positive, $Z_{\Phi}^{\prime}$ is compact and

$$
\left[R R_{K}^{J}(M, \mathcal{F}, \Phi)\right]^{K}=\left[R R_{K^{\prime}}^{J^{\prime}}\left(M^{\prime}, \mathcal{F}^{\prime}, \Phi^{\prime}\right)\right]^{K^{\prime}}
$$

Let us summarize what we have proved in this section for a line bundle $L \rightarrow M$ on an almost complex manifold $(M, J)$. Let $(\Omega, \Phi)$ be a weak 
Hamiltonian structure on $M$ such that $(\Omega, J)$ is adapted and $Z_{\Phi}$ is compact. Then the relation $\left[R R_{K}^{J}(M, L, \Phi)\right]^{K}=\left[R R_{K}^{J}\left(M, L, \Phi^{-1}(0), \Phi\right)\right]^{K}$ holds in two cases:

1. $L$ is a $\Phi$-moment line bundle,

2. $L$ is weakly $\Phi$-positive, $\Omega$ is non-degenerate and $\Phi^{-1}(0) \neq \emptyset$.

\subsection{Proofs}

We now concentrate on the proofs of Lemma 10.4, Proposition 10.3 and Theorems $10.6-10.9$.

Let $\beta \in \mathfrak{k} \backslash\{0\}$. If $m \in M^{\beta} \cap \Phi^{-1}(\beta)$, we have $\mathfrak{k}_{m} \subset \mathfrak{k}_{\beta}$ and then the map $X \mapsto X \cdot m,\left.\mathfrak{q}_{\beta} \rightarrow \mathcal{N}\right|_{m} \subset \mathrm{T}_{m} M$, is injective, $\mathbb{R}$-linear and equivariant relatively to the action of $\mathcal{L}(\beta)$. We consider the map

$$
\mathrm{p}_{m}:\left.\mathfrak{q}_{\beta} \rightarrow \mathcal{N}\right|_{m} ^{\beta,+}
$$

which is the composition of the injective map $\left.\mathfrak{q}_{\beta} \hookrightarrow \mathcal{N}\right|_{m}$ with the projection $\left.\left.\mathcal{N}\right|_{m} \rightarrow \mathcal{N}\right|_{m} ^{\beta,+}$. The map $\mathrm{p}_{m}$ is complex linear if both vector spaces $\mathfrak{q}_{\beta}$ and $\left.\mathcal{N}\right|_{m}$ are endowed with the complex structure $J_{\beta}$.

Lemma 10.4 is a consequence of

Lemma 10.10 The map $\mathrm{p}_{m}$ is injective for any $m \in \Phi^{-1}(\beta) \cap M^{\beta}$.

Proof. Consider the symmetric $\mathbb{R}$-bilinear form

$$
H_{m}(v, w)=\Omega_{m}(v, \mathcal{L}(\beta) w)
$$

on $\left.\mathcal{N}\right|_{m}$. By our condition on $\Omega, H_{m}(v, v) \leq 0$ on $\left.\mathcal{N}_{J_{\beta},-}\right|_{m}$. In contrast, on the subspace $\left.\mathfrak{q} \cdot m \subset \mathcal{N}\right|_{m}$, the quadratic form $H_{m}$ is positive definite. Indeed $H_{m}(X \cdot m, X \cdot m)=(\Phi(m),[X,[\Phi(m), X]])=\|[\beta, X]\|^{2}$. Thus $\mathfrak{q} \cdot m$ does not intersect $\left.\mathcal{N}_{J_{\beta},-}\right|_{m}$, and then $\mathrm{p}_{m}$ is injective.

Remark 10.11 The proof above shows that if $\mathrm{p}_{m}$ is bijective, then $\mathfrak{q} \cdot m$ is a maximal subspace of $\left.\mathcal{N}\right|_{m}$ where the quadratic form associate to $H_{m}$ is definite positive.

The injectivity of the complex linear map $\mathrm{p}_{m}$ implies that $\frac{1}{i} \mathcal{L}(\beta) \geq 0$ on $\operatorname{det}\left(\left.\mathcal{N}_{J_{\beta},+}\right|_{m}\right) \otimes \operatorname{det}\left(\mathfrak{q}_{J_{\beta}}\right)^{-1}$, and for a connected component $\mathcal{X}$ of $M^{\beta}$ intersecting $\Phi^{-1}(\beta)$ we have

$$
\mathcal{X} \subset M_{o}^{\beta} \quad \Longleftrightarrow \quad \mathcal{L}(\beta)=0 \text { on } \operatorname{det}\left(\mathcal{N}_{J_{\beta},+} \mid \mathcal{X}\right) \otimes \operatorname{det}\left(\mathfrak{q}_{J_{\beta}}\right)^{-1}
$$


Moreover, when $m$ is on a neighborhood $\Phi^{-1}(\beta) \cap M_{o}^{\beta}$ of $\Phi^{-1}(\beta) \cap M_{o}^{\beta}$, the map $\mathrm{p}_{m}$ stay injective and realize a trivialization of the $\operatorname{bundle} \operatorname{det}\left(\mathcal{N}_{J_{\beta},+}\right) \otimes$ $\operatorname{det}\left(\mathfrak{q}_{J_{\beta}}\right)^{-1}$.

Let us determine the Clifford bundle $\mathbb{d}_{\beta}(\mathcal{E})$ associated to the $\operatorname{Spin}^{c}$ bundle $\mathcal{E}=\bigwedge_{J} \mathrm{~T} M$ on $M$. By definition, we have $\left.\mathcal{E}\right|_{M^{\beta}}=\bigwedge \overline{\mathcal{N}_{J_{\beta}}} \otimes \mathbb{d}_{\beta}(\mathcal{E})$.

Since $\mathcal{N}_{J_{\beta}} \simeq \mathcal{N}_{J_{\beta},+} \oplus \overline{\mathcal{N}_{J_{\beta},-}}$ as complex vector bundles, we have

$$
\begin{aligned}
\bigwedge \overline{\mathcal{N}_{J_{\beta}}} & \simeq \bigwedge \overline{\mathcal{N}_{J_{\beta},+}} \otimes \bigwedge \mathcal{N}_{J_{\beta},-} \\
& \simeq(-1)^{\operatorname{rank} \mathcal{N}_{J_{\beta},+}} \operatorname{det}\left(\mathcal{N}_{J_{\beta},+}\right)^{-1} \otimes \bigwedge \mathcal{N}_{J_{\beta},+} \otimes \bigwedge \mathcal{N}_{J_{\beta},-} \\
& \simeq(-1)^{\operatorname{rank} \mathcal{N}_{J_{\beta},+}} \operatorname{det}\left(\mathcal{N}_{J_{\beta},+}\right)^{-1} \otimes \bigwedge_{J} \mathcal{N}
\end{aligned}
$$

as graded Spin ${ }^{c}$-bundles on $\mathcal{N} \rightarrow M^{\beta}$. On the other hand we have also $\left.\bigwedge_{J} \mathrm{~T} M\right|_{M^{\beta}} \simeq \bigwedge_{J} \mathrm{~T} M^{\beta} \otimes \bigwedge_{J} \mathcal{N}$. This shows that

$$
\mathbb{d}_{\beta}(\mathcal{E}):=(-1)^{\mathrm{rank} \mathcal{N}_{J_{\beta},+}} \bigwedge_{J} \mathrm{~T} M^{\beta} \otimes \operatorname{det}\left(\mathcal{N}_{J_{\beta},+}\right) .
$$

At this stage we have proved Proposition 10.3: The Clifford bundle $\mathcal{E}=$ $\bigwedge_{J} \mathrm{~T} M$ is weakly $\Phi$-positive since for any connected component $\mathcal{X}$ of $M^{\beta}$ intersecting $\Phi^{-1}(\beta)$ we have $\frac{1}{i} \mathcal{L}(\beta) \geq 0$ on $\left.\mathbb{d}_{\beta}(\mathcal{E})\right|_{\mathcal{X}} \otimes \operatorname{det}\left(\mathfrak{q}_{J_{\beta}}\right)^{-1}$ as $\frac{1}{i} \mathcal{L}(\beta) \geq$ 0 on $\operatorname{det}\left(\mathcal{N}_{J_{\beta},+}\right) \otimes \operatorname{det}\left(\mathfrak{q}_{J_{\beta}}\right)^{-1}$ restricted to $\mathcal{X}$.

Moreover we compute that

$$
\begin{aligned}
\mathcal{E}_{[\beta]} & :=(-1)^{\operatorname{dim} \mathfrak{q}_{J_{\beta}}\left[\mathbb{d}_{\beta}(\mathcal{E}) \otimes \operatorname{det}\left(\mathfrak{q}_{J_{\beta}}\right)^{-1}\right]^{\beta}} \\
& =(-1)^{n_{\beta}} \mathbb{D}_{\beta} \otimes \bigwedge_{J} \mathrm{~T} M^{\beta}
\end{aligned}
$$

where $n_{\beta}=\operatorname{dim}_{\mathbb{C}} \mathfrak{q}_{J_{\beta}}-\operatorname{rank}_{\mathbb{C}} \mathcal{N}_{J_{\beta},+}$ and

$$
\mathbb{D}_{\beta}=\left[\operatorname{det}\left(\mathcal{N}_{J_{\beta},+}\right) \otimes \operatorname{det}\left(\mathfrak{q}_{J_{\beta}}\right)^{-1}\right]^{\beta} .
$$

Two cases hold for a connected component $\mathcal{X}$ of $M^{\beta}$ intersecting $\Phi^{-1}(\beta)$. Thanks to the discussion above (see (10.34)), we see that either $\mathcal{X} \nsubseteq M_{o}^{\beta}$ and then $\left.\mathbb{D}_{\beta}\right|_{\mathcal{X}}=[0]$, or $\mathcal{X} \subset M_{o}^{\beta}$ and then $n_{\beta}=0$ on $\mathcal{X}$ and $\left.\mathbb{D}_{\beta}\right|_{\mathcal{U}} \simeq \mathcal{U} \times \mathbb{C}$ on a neighborhood $\mathcal{U}$ of $\mathcal{X} \cap \Phi^{-1}(\beta)$ in $\mathcal{X}$.

Finally we see that Theorem 10.6 is a consequence of Theorem 9.6. If we work with the Clifford bundle $\mathcal{E}^{\prime}=\bigwedge_{J} \mathrm{~T} M \otimes \mathcal{F}$, we have $\left.\mathcal{E}_{[\beta]}^{\prime}\right|_{\mathcal{X}}=[0]$ unless $\mathcal{X}$ is contained in $M_{o}^{\beta}$, and we have $\left.\mathcal{E}_{[\beta]}^{\prime}\right|_{M_{o}^{\beta}} \simeq \bigwedge_{J} \mathrm{~T} M_{o}^{\beta} \otimes\left[\left.\mathcal{F}\right|_{M_{o}^{\beta}}\right]^{\beta}$ in a neighborhood of $M_{o}^{\beta} \cap \Phi^{-1}(\beta)$. So we can conclude. 
We now concentrate ourselves on the proof of Theorem 10.7 and Theorem 10.9. First we prove the preliminary lemma, Lemma 10.8. Let $K^{0}$ be the connected component of $K$. We assume $M=K M^{0}$ where $M^{0}$ is connected and stable by $K^{0}$. Let $T$ be the maximal torus of $K^{0}$ with Lie algebra $\mathfrak{t}$. We make the choice of a Weyl chamber $\mathfrak{t}_{>0}^{*}$. The Convexity Theorem $[2,15,21,23]$ is telling us that the set $\Delta(M)=\Phi(M) \cap \mathfrak{t}_{\geq 0}^{*}$ is a convex polytope, called the Kirwan polytope. So there is a unique point $\beta_{\min } \in$ $\Delta(M)$ at minimum distance of the origin. So $K \beta_{\min }$ is the unique orbit at minimal distance in $\Phi(M)$.

Let $\beta_{\min } \in \Phi(M)$ at minimum distance of the origin. Equation $d\|\Phi\|^{2}=$ $2 \iota\left(\kappa_{\Phi}\right) \Omega$ shows that $\Phi^{-1}\left(\beta_{\min }\right)$ is contained in $M^{\beta_{\min }}=M^{\prime}$. Let $K^{\prime}$ be $K_{\beta_{\text {min }}}$. We notice that $M^{\prime}$ is a $K^{\prime}$-Hamiltonian manifold with moment map $\Phi_{M^{\prime}}$, and $J$ induces an invariant almost complex structure $J^{\prime}$ on $M^{\prime}$. We have the following basic properties whose proof is left to the reader.

Lemma 10.12 • The data $\left(\Omega^{\prime}, J^{\prime}\right)$ is adapted. - The map $\Phi_{M^{\prime}}$ is proper and $Z_{\Phi_{M^{\prime}}}$ is compact. • If $\mathcal{F}$ is weakly $\Phi$-positive, then $\mathcal{F}_{M^{\prime}}$ is weakly $\Phi_{M^{\prime-}}$ positive.

Let us go back to the proofs of Theorem 10.7 and Theorem 10.9.

Using Theorem 10.6, and Lemma 10.8 we are left to prove the following

Lemma 10.13 Let $\beta \in \mathcal{B} \backslash\{0\}$. The submanifold $M_{o}^{\beta}$ is non-empty if and only if $\beta=\beta_{\min }$.

Thus, let $\beta \in \mathcal{B}$ and consider $m \in \Phi^{-1}(\beta) \cap M^{\beta}$ such that the real rank of $\mathcal{N}_{J_{\beta},+}$ is equal to $\operatorname{dim}_{\mathbb{R}} \mathfrak{q}$. We want to prove that $\beta=\beta_{\text {min }}$. For that, we can only consider the action of the connected group $K^{0}$ on a connected component $M^{0}$ of $M$. Changing locally of notations for the aim of this proof, we now assume $K$ and $M$ connected. Let us use the notations of the proof of Lemma 10.10. As $m \in M^{\beta}, m$ is a critical point of the $\operatorname{map}(\Phi, \beta): M \rightarrow \mathbb{R}$. Its Hessian $H_{m}$ at $m$ is the symmetric bilinear form defined in (10.33). The fact that the 2 -form $\Omega$ is symplectic implies that $H_{m}$ is non-degenerate on $\left.\mathcal{N}\right|_{m}$.

Let $U$ be the connected component of the set of elements $\xi$ in $\mathfrak{k}_{\beta}$ such that $\mathfrak{k}_{\xi} \subset \mathfrak{k}_{\beta}$. This is an open set in $\mathfrak{k}_{\beta}$ containing $\beta$. The subset $Y=\Phi^{-1}(U)$ is connected, and is a $K_{\beta}$-symplectic submanifold of $M$ such that $K Y \subset M$ is a dense open subset of $M$.

Let $\mathcal{N}^{Y}$ be the normal bundle of $Y^{\beta}$ in $Y$. At the level of tangent spaces 
we have the decompositions

$$
\begin{aligned}
\mathrm{T}_{m} M & =\left.\mathrm{T}_{m} M^{\beta} \oplus \mathcal{N}\right|_{m} \\
& =\left.\mathrm{T}_{m} Y^{\beta} \oplus \mathfrak{q} \cdot m \oplus \mathcal{N}^{Y}\right|_{m}
\end{aligned}
$$

that are orthogonal with respect to $\Omega_{m}$ (and also under $H_{m}$ ).

Now, if the map $\mathrm{p}_{m}$ is an isomorphism, $\mathfrak{q} \cdot m$ is a maximal subspace of $\left.\mathcal{N}\right|_{m}$ where the quadratic form associate to $H_{m}$ is definite positive (see Remark 10.11). As $H_{m}$ is non-degenerate on $\left.\mathcal{N}^{Y}\right|_{m}$, necessarily $H_{m}(v, v)<0$ for any non-zero element $\left.v \in \mathcal{N}^{Y}\right|_{m}$. So, we have $(\Phi, \beta) \geq\|\beta\|^{2}$ on a neighborhood of $m$ in $Y$. In the symplectic setting, any function of the type $(\Phi, \beta)$ has a unique local minimum, thus $(\Phi, \beta) \geq\|\beta\|^{2}$ on $Y$, and consequently $\|\Phi\|^{2} \geq$ $\|\beta\|^{2}$ on $Y$. By $K$-invariance it implies that $\|\Phi\|^{2} \geq\|\beta\|^{2}$ on $M$. So $\beta=\beta_{\text {min }}$ as desired.

\section{3 $[Q, R]=0$ and semi-ample line bundles}

We define the notion of semi-ample line bundles in the almost complex setting.

Definition 10.14 Let $L$ be a $K$-equivariant line bundle on an almost complex manifold $(M, J)$. We say that $L$ is semi-ample, if there exists a $K$ invariant Hermitian connection with curvature two form $R_{L}=i \Omega_{L}$ such that $\Omega_{L}(v, J v) \geq 0$ for $v \in \mathrm{T} M$. The corresponding moment map $\Phi_{L}$ will be called adapted.

The following corollary is clear.

Corollary 10.15 Let $L$ be a $K$-equivariant semi-ample line bundle on a compact almost complex manifold $M$, with adapted moment map $\Phi_{L}$. Then

$$
\left[R R_{K}^{J}\left(M, L^{\otimes k}\right)\right]^{K}=\left[R R_{K}^{J}\left(M, L^{\otimes k}, \Phi_{L}^{-1}(0), \Phi_{L}\right)\right]^{K}
$$

for any $k \geq 1$. In particular, $\left[R R_{K}^{J}\left(M, L^{\otimes k}\right)\right]^{K} \neq 0$ for some $k \geq 1$ only if $0 \in \Phi_{L}(M)$.

This theorem is an analogue of the GIT construction. We will obtain more precise results on the description of $\left[R R_{K}(M, \mathcal{F})\right]^{K}$ via a moment map $\Phi$ if we can choose $(\Omega, \Phi)$ such that $\Omega$ is non degenerate, and $\mathcal{F}$ weakly $\Phi$ positive. This is the object of Section 12.4. 
Example 10.16 We follow the notations of Example 5.12. Let $\mathbb{F}:=K / T$ be the flag manifold equipped with an integrable complex structure. The line bundle $\left[\mathbb{C}_{\mu}\right]:=K \times_{T} \mathbb{C}_{\mu}$ associated to a weight $\mu \in \Lambda$ is semi-ample if and only if $\mu$ is dominant, and $\left[\mathbb{C}_{\mu}\right]$ is ample if and only if $\mu$ is regular dominant.

Example 10.17 The cotangent bundle $\mathrm{T}^{*} \tilde{K}$ of a compact Lie group $\tilde{K}$ is equipped with the symplectic form $\Omega=d \lambda$ where $\lambda$ is the Liouville 1-form (see section 8.5). Let $J$ be a almost complex structure on $\mathrm{T}^{*} \tilde{K}$ that is compatible with $\Omega$. Let us consider the trivial line bundle $[\mathbb{C}]=T^{*} \tilde{K} \times \mathbb{C}$ with the connection $\nabla:=d+i \lambda$. We see then that the two form $\Omega_{[\mathbb{C}]}:=\frac{1}{i} \nabla^{2}$ is equal to $\Omega$.

\section{A slice theorem for deformed symbol}

\subsection{An induction formula}

Let $H$ be a closed subgroup of $K$, and consider a $H$-invariant decomposition $\mathfrak{k}=\mathfrak{h} \oplus \mathfrak{q}$. Let $N^{\prime}$ be a $H$-manifold, and consider the $K$-manifold

$$
N=K \times_{H}\left(B_{\mathfrak{q}^{*}} \times N^{\prime}\right)
$$

where $B_{\mathfrak{q}^{*}} \subset \mathfrak{q}^{*}$ is a $H$-invariant ball centered at 0 . Then $N^{\prime}$ is a submanifold of $N$, and the normal bundle of $N^{\prime}$ in $N$ is isomorphic to the trivial bundle with fiber $\mathfrak{q} \oplus \mathfrak{q}^{*}:$ for $n^{\prime} \in N^{\prime}$, the element $X \in \mathfrak{q}$ defines the vector $\left.\frac{d}{d t}\right|_{t=0} e^{t X} \cdot\left[1,0, n^{\prime}\right] \in \mathrm{T}_{\left[1, n^{\prime}, 0\right]} N$.

The vector space $\mathfrak{q} \oplus \mathfrak{q}^{*}$ is identified to $\mathfrak{q}_{\mathbb{C}}$ by $X \oplus \xi \rightarrow X \oplus i \tilde{\xi}$ : here $\xi \in \mathfrak{q}^{*} \rightarrow \tilde{\xi} \in \mathfrak{q}$ is the isomorphism associated to an invariant scalar product on $\mathfrak{k}$. Let $S_{\mathfrak{q}}=\wedge \mathfrak{q}_{\mathbb{C}}$ be the spinor space for $\mathfrak{q}_{\mathbb{C}}$. Thus if $\mathcal{E}$ is a $K$-equivariant graded Clifford bundle on $N$, there exists a $H$-equivariant graded Clifford bundle $\mathcal{E}^{\prime}$ on $N^{\prime}$ such that

$$
\left.\mathcal{E}\right|_{N^{\prime}}=S_{\mathfrak{q}} \otimes \mathcal{E}^{\prime}
$$

Let $\Phi^{\prime}: N^{\prime} \rightarrow \mathfrak{h}^{*}$ be a $H$-equivariant map, and let $\Phi: N \rightarrow \mathfrak{k}^{*}$ be a $K$-equivariant map. We assume that these maps are linked by the following relations :

$$
\left\{\begin{array}{l}
\left.\Phi\right|_{N^{\prime}}=\Phi^{\prime}, \\
\Phi\left(\left[1 ; \xi, n^{\prime}\right]\right) \in \mathfrak{h}^{*} \Longleftrightarrow \xi=0 \\
\left(\Phi\left(\left[1 ; \xi, n^{\prime}\right]\right), \xi\right) \geq 0
\end{array}\right.
$$

for $\left(\xi, n^{\prime}\right) \in B_{\mathfrak{q}^{*}} \times N^{\prime}$.

The following induction result will be useful. 
Proposition 11.1 - The critical sets $Z_{\Phi} \subset N$ and $Z_{\Phi^{\prime}} \subset N^{\prime}$ are related by $Z_{\Phi}=K \times_{H}\left(\{0\} \times Z_{\Phi^{\prime}}\right)$.

- Let $Z$ be a compact component of $Z_{\Phi}$ and $Z^{\prime}$ its intersection with $N^{\prime}$. Then $Z^{\prime}$ is a compact component of $Z_{\Phi^{\prime}}$ and

$$
\mathcal{Q}_{K}(N, \mathcal{E}, Z, \Phi)=\operatorname{Ind}_{H}^{K}\left(\mathcal{Q}_{H}\left(N^{\prime}, \mathcal{E}^{\prime}, Z^{\prime}, \Phi^{\prime}\right)\right) .
$$

This leads to the relation $\left[\mathcal{Q}_{K}(N, \mathcal{E}, Z, \Phi)\right]^{K}=\left[\mathcal{Q}_{H}\left(N^{\prime}, \mathcal{E}^{\prime}, Z^{\prime}, \Phi^{\prime}\right)\right]^{H}$.

Proof. Let $\left(\xi, n^{\prime}\right) \in B_{\mathfrak{q}^{*}} \times N^{\prime}$. We identify $\mathrm{T}_{\left[1 ; \xi, n^{\prime}\right]} N$ to $\mathfrak{q} \oplus \mathfrak{q}^{*} \oplus \mathrm{T}_{n^{\prime}} N^{\prime}$. Let us write $\Phi\left(\left[1 ; \xi, n^{\prime}\right]\right)=\Phi_{\mathfrak{q}}\left(\xi, n^{\prime}\right) \oplus \Phi_{\mathfrak{h}}\left(\xi, n^{\prime}\right)$ in the decomposition $\mathfrak{k}=\mathfrak{h} \oplus \mathfrak{q}$ (and we have identified $\mathfrak{k}^{*}$ and $\mathfrak{k}$ ). We have

$$
\kappa_{\Phi}\left(\left[1 ; \xi, n^{\prime}\right]\right)=-\Phi_{\mathfrak{q}}\left(\xi, n^{\prime}\right) \oplus\left[\xi, \Phi_{\mathfrak{h}}\left(\xi, n^{\prime}\right)\right] \oplus-\Phi_{\mathfrak{h}}\left(\xi, n^{\prime}\right) \cdot n^{\prime} .
$$

From (11.36), we have that $\Phi_{\mathfrak{q}}\left(\xi, n^{\prime}\right)=0$ only if $\xi=0$. Hence the vector field $\kappa_{\Phi}$ vanishes at $\left[1 ; X, n^{\prime}\right]$ if and only if $\xi=0$ and $\kappa_{\Phi^{\prime}}\left(n^{\prime}\right)=-\Phi_{\mathfrak{h}}\left(0, n^{\prime}\right) \cdot n^{\prime}=0$. So if $Z$ a component of $Z_{\Phi}$, its intersection $Z^{\prime}$ with $\mathfrak{q} \times N^{\prime}$ is a component of $Z_{\Phi^{\prime}}$ such that $Z=K \times_{H}\left(\{0\} \times Z^{\prime}\right)$.

Consider a compact component $Z$ of $Z_{\Phi}$. Let $\mathcal{U}^{\prime}$ be a relatively compact neighborhood of $Z^{\prime}$ in $N^{\prime}$, so that $\mathcal{V}=B_{\mathfrak{q}} \times \mathcal{U}^{\prime}$ is a relatively compact neighborhood of $Z^{\prime}$ in $\mathfrak{q} \times M^{\prime}$. Let $\tau$ be the restriction of $\sigma(M, \mathcal{E}, \Phi)$ to $\mathrm{T}^{*} \mathcal{V}$. We are working on $K \times_{H} \mathcal{V}$, a small neighborhood of $Z=K Z^{\prime}$ in $M$, and by the induction formula of Proposition 4.7, we have

$$
\mathcal{Q}_{K}(M, \mathcal{E}, Z, \Phi)=\operatorname{Ind}_{H}^{K}\left(\operatorname{Index}_{H}^{\mathcal{V}}(\tau)\right) .
$$

Let us consider the injection $i: \mathcal{U}^{\prime} \rightarrow \mathcal{V}$. The symbol $\sigma^{\prime}=\sigma\left(\mathcal{U}^{\prime}, \mathcal{E}^{\prime}, \Phi^{\prime}\right)$ is a $H$-equivariant transversally elliptic symbol on $\mathcal{U}^{\prime}$. Its index is by definition $\mathcal{Q}_{H}\left(N^{\prime}, \mathcal{E}^{\prime}, Z^{\prime}, \Phi^{\prime}\right)$. We can construct the push-forward $i_{!}\left(\sigma^{\prime}\right)$ of the symbol $\sigma^{\prime} \in \mathbf{K}_{H}^{0}\left(\mathrm{~T}_{H}^{*} \mathcal{U}^{\prime}\right)$ in $\mathbf{K}_{H}^{0}\left(\mathrm{~T}_{H}^{*} \mathcal{V}\right)$ as defined in Section 5.4. Our proposition 11.1 will follow from the following formula and Theorem 5.9.

\section{Lemma 11.2}

$$
\tau=i_{!}\left(\sigma^{\prime}\right)
$$

Proof. By definition the symbol $i_{!}\left(\sigma^{\prime}\right)$ is equal to $\sigma^{\prime} \otimes \operatorname{Bott}\left(\mathfrak{q}_{\mathbb{C}}\right)$. For $\left(\xi, n^{\prime}\right) \in \mathfrak{q}^{*} \times N^{\prime}$, and $(q, \nu) \in \mathfrak{q} \times \mathrm{T}_{n^{\prime}}^{*} N^{\prime}$, we have

$$
i_{!}\left(\sigma^{\prime}\right)_{\left(\xi, n^{\prime}\right)}(q, \nu)=\mathbf{c}_{\mathcal{E}_{n^{\prime}}^{\prime}}\left(\tilde{\nu}-\kappa_{\Phi^{\prime}}\left(n^{\prime}\right)\right) \otimes \mathbf{c}_{S_{\mathfrak{q}}}(\tilde{\xi} \oplus i q) .
$$


We compute $\tau_{\left(X, n^{\prime}\right)}(q, \nu)$. Using Formula 11.37 we have

$$
\tau_{\left(\xi, n^{\prime}\right)}(q, \nu)=\mathbf{c}_{\mathcal{E}_{n^{\prime}}^{\prime}}\left(\tilde{\nu}+\Phi_{\mathfrak{h}}\left(\xi, n^{\prime}\right) \cdot n^{\prime}\right) \otimes \mathbf{c}_{S_{\mathfrak{q}}}\left(\Phi_{\mathfrak{q}}\left(\xi, n^{\prime}\right) \oplus i\left(q-\left[\xi, \Phi_{\mathfrak{h}}\left(\xi, n^{\prime}\right)\right]\right)\right) .
$$

For any $t \in[0,1]$, we define

$$
\begin{aligned}
A^{t}\left(\xi, n^{\prime}, q\right) & =t \Phi_{\mathfrak{q}}\left(\xi, n^{\prime}\right)+(1-t) \xi \oplus i\left(q-t\left[\xi, \Phi_{\mathfrak{h}}\left(\xi, n^{\prime}\right)\right]\right) \in \mathfrak{q}_{\mathbb{C}} \\
B^{t}\left(\xi, n^{\prime}\right) & =-t \Phi_{\mathfrak{h}}\left(\xi, n^{\prime}\right) \cdot n^{\prime}+(1-t) \kappa_{\Phi^{\prime}}\left(n^{\prime}\right) \in \mathrm{T}_{n^{\prime}} N^{\prime},
\end{aligned}
$$

and the symbol

$$
\tau_{\left(\xi, n^{\prime}\right)}^{t}(q, \nu)=\mathbf{c}_{\mathcal{E}_{n^{\prime}}^{\prime}}\left(\tilde{\nu}-B^{t}\left(\xi, n^{\prime}\right)\right) \otimes \mathbf{c}_{S_{\mathfrak{q}}}\left(A^{t}\left(\xi, n^{\prime}, q\right)\right) .
$$

We have $\left\|A^{t}\left(\xi, n^{\prime}, q\right)\right\|^{2}=\left\|t \Phi_{\mathfrak{q}}\left(\xi, n^{\prime}\right)+(1-t) \xi\right\|^{2}+\left\|\tilde{q}-t\left[\xi, \Phi_{\mathfrak{h}}\left(\xi, n^{\prime}\right)\right]\right\|^{2}$.

If $\left(\left(\xi, n^{\prime}\right),(q, \nu)\right)$ is in the support of $\tau^{t}$, necessarily $A^{t}\left(\xi, n^{\prime}, q\right)=0$. We use the conditions (11.36). As $\left(\xi, \Phi_{\mathfrak{q}}\left(\xi, n^{\prime}\right)\right)=\left(\Phi\left(\left[1 ; \xi, n^{\prime}\right]\right), \xi\right) \geq 0$, we see that $\left\|t \Phi_{\mathfrak{q}}\left(\xi, n^{\prime}\right)+(1-t) \xi\right\|^{2} \geq t^{2}\|\xi\|^{2}+(1-t)^{2}\left\|\Phi_{\mathfrak{q}}\left(\xi, n^{\prime}\right)\right\|^{2}$ and this last expression vanishes only when $\xi=0$. Thus, we see that, when $\left(\xi, n^{\prime}\right) \in B_{\mathfrak{q}} \times N^{\prime}$, the vector $A^{t}\left(\xi, n^{\prime}, q\right)$ vanishes if and only if $\xi=0$ and $q=0$. And for any $t \in[0,1], B^{t}\left(0, n^{\prime}\right)=\kappa_{\Phi^{\prime}}\left(n^{\prime}\right)$.

Thus $\tau^{t}$ is an homotopy of transversally elliptic symbol on $B_{\mathfrak{q}} \times N^{\prime}$. Hence $\tau^{1}=\tau$ defines the same $\mathbf{K}$-theory class than $\tau^{0}=i_{!}\left(\sigma^{\prime}\right)$.

In the next subsection, we give a first application of this formula.

\subsection{The shifting formula}

We consider a $K$-manifold $M$, oriented and of even dimension, that is equipped with a $K$-equivariant Clifford bundle $\mathcal{G}$. Let $\Phi_{M}: M \rightarrow \mathfrak{k}^{*}$ be an equivariant map. Let $a \in \mathfrak{k}^{*}$, and let $H=K_{a}$ be the stabilizer subgroup of $a$. On the coadjoint orbit $K a$, consider the inclusion $\Phi_{a}: K a \hookrightarrow \mathfrak{k}^{*}$. We assume that $K a$ is equipped with a $K$-equivariant Clifford bundle $\mathcal{F}$. On the product $M \times K a$, consider the map $\Phi=\Phi_{M}-\Phi_{a}$.

In this section we assume that $\Phi^{-1}(0)$ is a compact component of $Z_{\Phi}$ : this property holds when $\Phi_{M}$ is a moment map and $M$ is compact.

Consider

$$
\mathcal{Q}_{K}\left(M \times K a, \mathcal{G} \otimes \mathcal{F}, \Phi^{-1}(0), \Phi\right)
$$

the equivariant index localized near the component $\Phi^{-1}(0) \subset M \times K a$.

We look at a small $H$-invariant open neighborhood $B$ of $a$ in $\mathfrak{h}^{*}$ such that $Y=\Phi_{M}^{-1}(B) \subset M$ is a slice at $a \in \mathfrak{k}^{*}$ for the map $\Phi_{M}$. Then $Y$ is a 
$H$-invariant manifold of $M$ such that $\Phi_{M}(Y) \subset \mathfrak{h}^{*}$ and such that $K \times_{H} Y$ is diffeomorphic to a invariant open neighborhood of $\Phi_{M}^{-1}(K a)$. We denote by $\Phi_{Y}$ the restriction of the map $\Phi_{M}$ to $Y$.

The element $a \in \mathfrak{k}$ acts by an invertible skew-symmetric transformation on $\mathfrak{k} / \mathfrak{k}_{a}$. We denote by $\mathfrak{q}_{J_{a}}$ the vector space $\mathfrak{k} / \mathfrak{k}_{a}$ equipped with the complex structure $J_{a}$, We write $\left.\mathcal{G}\right|_{Y}=\mathcal{G}_{d Y} \otimes \wedge \mathfrak{q}_{J_{a}}$ where $\mathcal{G}_{d Y}$ is a $H$-equivariant Clifford bundle on $Y$, and $\left.\mathcal{F}\right|_{\{a\}}=\bigwedge \overline{\mathfrak{q}_{J_{a}}} \otimes F$ where $F \in R(H)$ (here $\{a\}$ is the base point of $K a$ ).

Proposition 11.3 For any equivariant Clifford bundles $\mathcal{G} \rightarrow M$ and $\mathcal{F} \rightarrow$ $K a$, and any equivariant map $\Phi(k, \xi)=\Phi_{M}(m)-\xi$ such that $\Phi^{-1}(0)$ is a compact component of $Z_{\Phi}$, we have

$\mathcal{Q}_{K}\left(M \times K a, \mathcal{G} \otimes \mathcal{F}, \Phi^{-1}(0), \Phi\right)=\operatorname{Ind}_{H}^{K}\left(\mathcal{Q}_{H}\left(Y, \mathcal{G}_{d Y}, \Phi_{Y}^{-1}(a),\left(\Phi_{Y}-a\right)\right) \otimes F\right)$.

Proof. We show that we are in the situation of Subsection 11.1. A neighborhood of $\Phi^{-1}(K a)$ in $M$ is of the form $K \times_{H} Y$. Let us consider the diffeomorphism $\varphi: K \times_{H}(K a \times Y) \rightarrow K a \times\left(K \times_{H} Y\right)$ defined by $\varphi([k ; \xi, y])=(k \xi, k y)$. Through $\varphi$, the map $\Phi$ becomes on $K \times_{H}(K a \times Y)$ the map $\tilde{\Phi}([k ; \xi, y])=k\left(\Phi_{Y}(y)-\xi\right)$, and the set $\tilde{\Phi}^{-1}(0)$ is equal to $K \times$ $\left(\{a\} \times \phi^{-1}(a)\right)$.

We are then working on a $H$-invariant neighborhood of $\{a\}$ in $K a$. This neighborhood can indeed be parameterized by $\mathfrak{q}^{*}$ through the map $\xi \rightarrow e^{\tilde{\xi}} a$. So we work with the $K$-manifold $N=K \times_{H}\left(B_{\mathfrak{q}^{*}} \times Y\right)$ and the $H$-manifold $N^{\prime}=Y$. The equivariant maps are $\Phi([k ; \xi, y]):=k\left(\Phi_{Y}(y)-e^{\tilde{\xi}} a\right)$ on $N$ and $\Phi^{\prime}=\Phi_{Y}-a$ on $Y$. We check easily that relations (11.36) hold if the ball $B_{\mathfrak{q}^{*}}$ is small enough. Indeed, $\left(e^{\tilde{\xi}} a, \tilde{\xi}\right)=0$ for any $\xi \in \mathfrak{q}^{*}$, and for small $\xi$ the term $e^{\tilde{\xi}} a=a+[\tilde{\xi}, a]+O\left(\|\tilde{\xi}\|^{2}\right)$ belongs to $\mathfrak{h}$ only if $\xi=0$.

At the level of Clifford bundles, if one considers $\mathcal{E}:=\mathcal{G} \otimes \mathcal{F}$, the corresponding $\mathcal{E}^{\prime}$ on $Y$ is $\mathcal{E}^{\prime}:=\mathcal{G}_{d Y} \otimes F$ since the Clifford bundle $S_{\mathfrak{q}_{a}}$ is equal to $\bigwedge \mathfrak{q}_{J_{a}} \otimes \bigwedge \overline{\mathfrak{q}_{J_{a}}}$. So we are in the setting of Proposition 11.1 and we can conclude.

\section{The Hamiltonian setting}

Let us assume that our $K$-manifold $M$ is provided with a Hamiltonian structure $(\Phi, \Omega): \Omega$ is a non degenerate closed 2-form on $M$ and the moment map $\Phi: M \rightarrow \mathfrak{k}^{*}$ and $\Omega$ are related by the Kostant relations (6.14). Then we can always choose an invariant almost complex structure $J$ on $M$ such that 
$\Omega(v, J w), v, w \in \mathrm{T} M$ is a Riemannian metric on $M$ : such almost complex structure $J$ is called compatible with $\Omega$.

Consider the case where $M$ compact. In this context, the representation $\mathcal{Q}_{K}\left(M, \bigwedge_{J} \mathrm{~T} M \otimes \mathcal{F}\right)$ does not depend on the choice of $J$ since the set of $\Omega$ compatible almost complex structures is contractible, so, given $\Omega$, we denote it by $R R_{K}(M, \mathcal{F})$ whenever the almost complex structure $J$ is compatible with the given choice of $\Omega$.

We can make use of the result of Theorem 10.7, since our hypothesis " $J$ compatible with $\Omega$ " is stronger than the hypothesis " $(\Omega, J)$ is adapted". In fact we will gain much more, as we will be able to compute geometrically the multiplicities of $R R_{K}(M, L)$ when $L$ is a $\Phi$-moment bundle : it is the heart of the $[Q, R]=0$ theorem of Meinrenken-Sjamaar that is explained in the next section.

Our proof of the Meinrenken-Sjamaar Theorem relies on the Witten deformation argument presented in the previous section. We attach a localized Riemann-Roch character

$$
R R_{K}\left(M, \mathcal{F}, Z_{\beta}, \Phi\right)=\mathcal{Q}_{K}\left(M, \bigwedge_{J} \mathrm{~T} M \otimes \mathcal{F}, Z_{\beta}, \Phi\right)
$$

to each component $Z_{\beta}$ of $Z_{\Phi}[32,35,24]$, where $J$ is an almost complex structure compatible with $\Omega$. In this way, we decompose the character $R R_{K}(M, \mathcal{F})$ in sum of (infinite) dimensional representations, while Meinrenken-Sjamaar decomposes $R R_{K}(M, \mathcal{F})$ in sums of (finite) dimensional representations by cutting the Kirwan polytope in small polytopes. In both of these methods, when $\mathcal{F}$ is weakly $\Phi$-positive, the study of $\left[R R_{K}(M, \mathcal{F})\right]^{K}$ is reduced to the study of $M$ around $\Phi^{-1}(0)$, and this can be done explicitly, at least in the case where 0 is a quasi-regular value. The use of the compact connected component $\Phi^{-1}(0)$ of the zeroes of the Kirwan vector field $\kappa_{\Phi}$ replaces the compactification of a neighborhood of $\Phi^{-1}(0)$ by cutting used in Meinrenken-Sjamaar.

When $M$ is non-compact, we can also study the localized Riemann-Roch character $R R_{K}(M, \mathcal{F}, \Phi)$ when the set $Z_{\Phi}$ is proper [35, 24]. In Section 13 we will concentrate ourselves to the case of an Hamiltonian action of $\tilde{K} \times K$ on the cotangent bundle $\mathrm{T}^{*} \tilde{K}$.

\section{1 $[Q, R]=0$ Theorem of Meinrenken-Sjamaar}

Assume in this subsection that $K$ is a compact connected Lie group, and let $T$ be its maximal torus. Let $\Lambda \subset \mathfrak{t}^{*}$ be the weight lattice of $T$, and consider the set of dominant weights $\Lambda_{\geq 0}=\Lambda \cap \mathfrak{t}_{\geq 0}^{*}$ that parameterizes 
the irreducible representations of $K:$ for $\mu \in \Lambda_{\geq 0}$, we denote by $V_{\mu}^{K}$, the irreducible representation with highest weight $\mu$.

For the remaining part of this section we assume that $M$ is compact and connected, and we denote $\Delta(M):=\Phi(M) \cap \mathfrak{t}_{\geq 0}^{*}$ the Kirwan polytope.

Let $L \rightarrow M$ be a $\Phi$-moment bundle. Write

$$
R R_{K}(M, L)=\sum_{\mu \in \Lambda_{\geq 0}} \mathrm{~m}_{\mu}(L) V_{\mu}^{K} .
$$

We will explain how to compute geometrically the multiplicity $\mathrm{m}_{\mu}(L)$ of the representation $V_{\mu}^{K}$ in $R R_{K}(M, L)$ in terms of the fibers of the moment map.

Definition 12.1 For any $a \in \mathfrak{k}^{*}$, we define the topological space $M_{a}:=$ $\Phi^{-1}(K a) / K$.

We say that $M_{a}$ is the reduced space of $M$ at $a$. In particular, the reduced space $M_{\text {red }}=\Phi^{-1}(0) / K$ is denoted $M_{0}$.

Let us recall the notion of quasi-regular value of $\Phi$.

Definition 12.2 An element $a \in \mathfrak{k}^{*}$ is a quasi-regular value of $\Phi$ if $a \in$ $\Phi(M)$ and the $K$-orbits in $\Phi^{-1}(K a)$ all have the same dimension.

Equivalently, $a$ is a quasi-regular value if there exists a subalgebra $\mathfrak{s} \subset$ $\mathfrak{k}$ such that $\Phi^{-1}(K a)$ is contained in the infinitesimal orbit type stratum $M_{(\mathfrak{s})}=\left\{m \in M,\left(\mathfrak{k}_{m}\right)=(\mathfrak{s})\right\}$. As we will see, this implies that $\Phi^{-1}(K a)$ is a smooth $K$-manifold. Furthermore, if $a$ is a quasi-regular value, then the orbifold stratification of $M_{a}:=\Phi^{-1}(K a) / K$ consists of one piece only, and $M_{a}$ is therefore a symplectic orbifold.

Assume that a dominant weight $\mu$ is a quasi-regular value of $\Phi$. We can then consider the orbifold line bundle over $M_{\mu}$,

$$
L_{\mu}:=\left(\left.L\right|_{\Phi^{-1}(\mu)} \otimes \mathbb{C}_{-\mu}\right) / K_{\mu},
$$

and we may define the Riemann-Roch number $R R\left(M_{\mu}, L_{\mu}\right)$.

In general, we consider quasi-regular values $a$ of $\Phi$ close to $\mu$, and we can, as we will see, define the orbifold line bundle

$$
L_{a, \mu}:=\left(\left.L\right|_{\Phi^{-1}(a)} \otimes \mathbb{C}_{-\mu}\right) / K_{a},
$$

over the symplectic orbifold $M_{a}=\Phi^{-1}(a) / K_{a}$. The line orbi-bundle $L_{a, \mu}$ is well defined since for $a$ close enough to $\mu$, the action of $\mathfrak{k}_{m}$ on $\left.L\right|_{m} \otimes \mathbb{C}_{-\mu}$ is trivial for any $m \in \Phi^{-1}(a)$ (see Lemma 12.20).

We can now state the Meinrenken-Sjamaar Theorem [29]. 
Theorem 12.3 Let $\mu \in \Lambda_{\geq 0}$.

- If $\mu \notin \Delta(M)$, then $\mathrm{m}_{\mu}(L)=0$.

- If $\mu \in \Delta(M)$, then $\mathrm{m}_{\mu}(L)=R R\left(M_{a}, L_{a, \mu}\right)$ for any quasi-regular value a of $\Phi$ that is close enough to $\mu$.

We can restate this result by defining Riemann-Roch numbers on (singular) symplectic reduced spaces as follows.

Definition 12.4 Let $L$ be a $\Phi$-moment bundle. For any dominant weight $\mu \in \Lambda_{\geq 0}$, the Riemann-Roch number $R R\left(M_{\mu}, L_{\mu}\right)$ is defined by the following dichotomy

$R R\left(M_{\mu}, L_{\mu}\right)= \begin{cases}0 & \text { if } \mu \notin \Delta(M), \\ R R\left(M_{a}, L_{a, \mu}\right) & \text { if } \mu \in \Delta(M) \text { and } a \text { is a quasi regular } \\ \text { value close enough } & \text { to } \mu .\end{cases}$

The $[Q, R]=0$ Theorem of Meinrenken-Sjamaar says that for a $\Phi$ moment bundle $L$, the multiplicity $\mathrm{m}_{\mu}(L)$ of the representation $V_{\mu}^{K}$ in $R R_{K}(M, L)$ is equal to $R R\left(M_{\mu}, L_{\mu}\right)$.

\subsection{Localization on $\Phi^{-1}(0)$}

In this section, we consider a $K$-Hamiltonian manifold $(M, \Omega, \Phi)$ not necessarily compact nor connected, and the group $K$ is not supposed connected. We study the character $R R_{K}\left(M, \mathcal{F}, \Phi^{-1}(0), \Phi\right)$, in the case where 0 is a quasi-regular value of $\Phi$ and the manifold $\Phi^{-1}(0)$ is compact.

\subsubsection{Regular case}

We suppose here that 0 is a regular value of $\Phi$. The group $K$ acts infinitesimally freely on the compact submanifold $P=\Phi^{-1}(0)$, and $M_{0}:=P / K$ is a compact orbifold equipped with an induced symplectic form $\Omega_{0}$. We denote $\pi: P \rightarrow P / K=M_{0}$ the projection.

Proposition 12.5 For any equivariant vector bundle $\mathcal{F} \rightarrow M$ we have

$$
\left[R R_{K}\left(M, \mathcal{F}, \Phi^{-1}(0), \Phi\right)\right]^{K}=R R\left(M_{0}, \mathcal{F}_{0}\right)
$$

where $\mathcal{F}_{0}=\left.\mathcal{F}\right|_{P} / K$. 
Proof. Let $\mathcal{E}:=\bigwedge_{J} \mathrm{~T} M$ be the Clifford bundle associated to a compatible almost complex structure $J$. We consider the restriction of the tangent bundle $\mathrm{T} M$ on the submanifold $P$. Let $\left.[\mathfrak{k}] \subset \mathrm{T} M\right|_{P}$ be the subbundle which is the image of the map $(m, X) \in P \times \mathfrak{k} \mapsto X \cdot m \in \mathrm{T}_{m} P \subset \mathrm{T}_{m} M$. We consider the subbundle $[\mathfrak{k}] \oplus J[\mathfrak{k}]$ of $\left.\mathrm{T} M\right|_{P}$ that is canonically isomorphic to $\left[\mathfrak{k}_{\mathbb{C}}\right]$. Let $\left.E \subset \mathrm{T} M\right|_{P}$ be the orthogonal of $[\mathfrak{k}] \oplus J[\mathfrak{k}]$ relatively to the symplectic form. We see that $E \rightarrow P$ is a symplectic bundle isomorphic to the pull-back $\pi^{*} \mathrm{~T} M_{0}$, and that $J$ induces an almost complex structure $J_{0}$ on $E$ compatible with $\pi^{*}\left(\Omega_{0}\right)$. We see then that $\left.\mathcal{E}\right|_{P} \simeq \bigwedge \mathfrak{E}_{\mathbb{C}} \otimes \mathcal{E}_{0}$ with $\mathcal{E}_{0}=\pi^{*}\left(\bigwedge_{J_{0}} \mathrm{~T} M_{0}\right)$. Our result follows then from Theorem 8.3.

\subsubsection{Reduction in stage}

In this section, we explore the case of reduction in stage. Suppose that we have a Hamiltonian action of the compact Lie group $G \times K$ on the manifold $(M, \Omega)$. Let $\Phi=\Phi_{G} \oplus \Phi_{K}: M \rightarrow \mathfrak{g}^{*} \oplus \mathfrak{k}^{*}$ be the corresponding moment map. We suppose that

- 0 is a regular value of $\Phi_{K}$,

- $K$ acts freely on $P:=\Phi_{K}^{-1}(0)$,

- $Z:=\Phi^{-1}(0)$ is compact.

We denote by $\pi: P \rightarrow M_{0}:=P / K$ the corresponding $G$-equivariant principal fibration. We denote by $\phi: M_{0} \rightarrow \mathfrak{g}^{*}$ the equivariant map induced by $\Phi_{G}$ : it is the moment map relative to the Hamiltonian action of $G$ on the symplectic manifold $\left(M_{0}, \Omega_{0}\right)$.

Proposition 12.6 For any equivariant vector bundle $\mathcal{F} \rightarrow M$, we have the following relation

$$
\begin{aligned}
& {\left[R R_{K \times G}\left(M, \mathcal{F}, \Phi^{-1}(0), \Phi\right)\right]^{K}=R R_{G}\left(M_{0}, \mathcal{F}_{0}, \phi^{-1}(0), \phi\right) \text { in } \hat{R}(G) .} \\
& \quad \text { Here } \mathcal{F}_{0}=\left(\left.\mathcal{F}\right|_{P}\right) / K .
\end{aligned}
$$

Proof. We follow the proof of Proposition 12.5. We analyze the symbol $\sigma(M, \Phi)(m, \nu):=\mathbf{c}_{m}\left(\tilde{\nu}-\kappa_{\Phi}(m)\right): \bigwedge_{J}^{\text {even }} \mathrm{T} M \rightarrow \bigwedge_{J}^{\text {odd }} \mathrm{T} M$ on the model $\hat{M}=P \times \mathfrak{k}^{*}$ in a neighborhood of $Z \times\{0\}$.

The choice of a $G \times K$-invariant connection 1 -form $\theta$ on the $K$-principal bundle $P \rightarrow M_{0}$ defines a projection map $\mathrm{T}_{p} P \rightarrow \mathfrak{k}$ which associates to a tangent vector its vertical part. This projection induces a map $j: P \rightarrow$ 
$\operatorname{hom}(\mathfrak{g}, \mathfrak{k})$, where the vertical part of the vector $X \cdot p \in \mathrm{T}_{p} P$ is equal to $j_{p}(X) \in \mathfrak{k}$ for any $(X, p) \in \mathfrak{g} \times P$.

The symplectic form can be taken as $\hat{\Omega}=\pi^{*} \Omega_{0}+d\langle\theta, \xi\rangle$, and the moment maps have the following expressions : for $(p, \xi) \in P \times \mathfrak{k}^{*}$

- $\Phi_{K}(p, \xi)=\xi$,

- $\Phi_{G}(p, \xi)=\phi(\bar{p})+j_{p}^{*}(\xi)$, where $\bar{p}=\pi(p)$.

We denote by $\kappa_{G}\left(\right.$ resp. $\left.\kappa_{\phi}\right)$ the Kirwan vector field on $P$ (resp. $M_{0}$ ) associated to the equivariant map $\Phi_{G}: P \rightarrow \mathfrak{g}^{*}$ (resp. $\left.\phi\right)$. The Kirwan vector field $\kappa_{\Phi}$ has the following expression $\kappa_{\Phi}=\kappa_{\Phi}^{\text {vert }}+\kappa_{\Phi}^{\text {hor }}$, where

- $-\kappa_{\Phi}^{v e r t}(p, \xi)=\left(I d+j_{p} j_{p}^{*}\right) \xi+j_{p} \phi(\bar{p})$,

- $-\kappa_{\Phi}^{\text {hor }}(p, \xi) \simeq-\kappa_{\phi}(\bar{p})+j_{p}^{*}(\xi) \cdot \bar{p}$ through the tangent map $\mathrm{T}_{p} \pi$.

Let $V \subset U$ be two invariant relatively compact neighborhoods of $Z=$ $\Phi_{G}^{-1}(0) \cap P$ in $P$ such that $\pi(\bar{U}) \cap\left\{\kappa_{\phi}=0\right\}=\phi^{-1}(0)$, and $\bar{V} \subset U$. The vector field $\kappa_{\phi}$ does not vanish on the compact set $\pi(\bar{U} \backslash V)$, hence we can choose the connection 1-form $\theta$ such that the vector field $\kappa_{G}$ is horizontal on $\bar{U} \backslash V$. In other words, the term $j_{p} \phi(\bar{p})$ vanishes for $p \in \bar{U} \backslash V$. Let $c>0$ such that $\left\|j_{p} \phi(\bar{p})\right\| \leq c$ on $V$. We deform $\Phi$ in $\Phi_{t}(p, \xi)=\xi \oplus\left(\phi(\bar{p})+t j_{p}(\xi)\right)$ for $t \in[0,1]$, and we denote $\kappa_{t}$ the corresponding vector field. The following lemma is an easy computation

Lemma 12.7 For any $t \in[0,1]$, the set $\left\{(p, \xi) \in U \times \mathfrak{k}^{*}, \kappa_{t}(p, \xi)=0\right\}$ is contained in the compact set $\bar{V} \times\{\|\xi\| \leq c\}$.

Thanks to the previous lemma, we see that the symbols $\sigma(M, \Phi)$ and $\sigma\left(M, \Phi_{0}\right)$ define the same class in $\mathbf{K}_{G \times K}^{0}\left(\mathrm{~T}_{G \times K}\left(U \times \mathfrak{k}^{*}\right)\right)$. Let $\sigma_{\text {hor }} \in$ $\mathbf{K}_{G \times K}^{0}\left(\mathrm{~T}_{G \times K} U\right)$ be the pullback of $\left.\sigma\left(M_{0}, \phi\right)\right|_{\pi(U)} \in \mathbf{K}_{G}^{0}\left(\mathrm{~T}_{G} \pi(U)\right)$.

For $m=(p, \xi) \in U \times \mathfrak{k}^{*}$ and $\nu:=\eta \oplus a \oplus b \in \mathrm{T}^{*}\left(U \times \mathfrak{k}^{*}\right)$, we have

$$
\sigma\left(M, \Phi_{0}\right)(m, \nu)=\sigma_{h o r}(p, \eta) \otimes \mathbf{c}_{\wedge \mathfrak{k}_{\mathbb{C}}}\left(\left(a+\gamma_{p}+\xi\right) \oplus i b\right)
$$

where $\gamma_{p}=j_{p} \phi(\bar{p})$. We see that the support of the symbol $\sigma\left(M, \Phi_{0}\right)$ intersected with $\mathrm{T}_{G \times K}^{*}\left(U \times \mathfrak{k}^{*}\right)$ is just equal to $Z \times\{0\}$.

We see also that for $t \in[0,1]$, the support of the symbol

$$
\sigma^{t}(m, \nu)=\sigma_{h o r}(p, \eta) \otimes \mathbf{c}_{\wedge \mathfrak{k}_{\mathbb{C}}}\left(\left(t\left(a+\gamma_{p}\right)+\xi\right) \oplus i b\right)
$$

intersected with $\mathrm{T}_{G \times K}^{*}\left(U \times \mathfrak{k}^{*}\right)$ remains equal to $Z \times\{0\}$. Thus we have proved that $\sigma(M, \Phi)$ is equal to $i_{!}\left(\sigma_{h o r}\right)$ in $\mathbf{K}_{G \times K}^{0}\left(\mathrm{~T}_{G \times K}\left(U \times \mathfrak{k}^{*}\right)\right)$. Our result follows from this fact. 


\subsubsection{Quasi regular case}

In this subsection, we study the character $R R_{K}\left(M, \mathcal{F}, \Phi^{-1}(0), \Phi\right)$, in the case where 0 is a quasi-regular value of $\Phi$.

In particular, we prove the first form of the $[Q, R]=0$ theorem.

Theorem 12.8 If 0 is a quasi-regular value of the moment map, and $L$ a $\Phi-m o m e n t$ bundle, then

$$
\left[R R_{K}(M, L)\right]^{K}=R R\left(M_{0}, L_{0}\right)
$$

Here $L_{0}=\left.L\right|_{\Phi^{-1}(0)} / K$.

Consider first the basic example of a Hermitian vector space $W$ with complex structure $J$ and Hermitian inner product $\mathrm{h}(v, w)$. Consider the symplectic form on $W$ given by $\Omega=-\operatorname{Im}(\mathrm{h})$. Thus $J$ is a complex structure adapted to $\Omega$. Let $K$ be a compact group acting by unitary transformations on $W$. Then $W$ is a $K$-Hamiltonian manifold with moment map $\langle\Phi(x), X\rangle=$ $\frac{i}{2} \mathrm{~h}(X \cdot x, x)$, for $X \in \mathfrak{k}, x \in W$.

Assume that $\Phi^{-1}(0)=\{0\}$ : in this case 0 is a quasi-regular value. Consider a complex representation space $F$ of $K$, and the $K$-equivariant vector bundle $[F]=W \times F$.

Proposition 12.9 Assume that $\Phi^{-1}(0)=0$. Then

$$
R R_{K}\left(W,[F], \Phi^{-1}(0), \Phi\right)=\operatorname{Sym}\left(W^{*}\right) \otimes F .
$$

Furthermore, $\left[\operatorname{Sym}\left(W^{*}\right)\right]^{K}=\mathbb{C}$.

Remark 12.10 The second claim follows from Mumford GIT theory. In spirit, this is the first case of the $[Q, R]=0$ theorem.

Proof. Let $x \in W$, and $\kappa_{\Phi}(x)=-\Phi(x) \cdot x$ be the Kirwan vector field associated to $\Phi$. Then $\kappa_{\Phi}$ vanishes only at 0 : indeed we have $\mathrm{h}\left(J x, \kappa_{\Phi}(x)\right)=$ $2\|\Phi(x)\|^{2}$.

Our deformed symbol is $\sigma(W, \Phi)(x, \nu)=\mathbf{c}\left(\tilde{\nu}-\kappa_{\Phi}(x)\right): \bigwedge^{\text {even }} W \longrightarrow$ $\bigwedge^{\text {odd }} W$, while Atiyah symbol $\operatorname{At}(\bar{W})$ is $\operatorname{At}(\bar{W})(x, \nu)=\mathbf{c}(\tilde{\nu}-J x): \bigwedge^{\text {even }} W \longrightarrow$ $\bigwedge^{\text {odd }} W$.

Let $t \in[0,1]$ and $\sigma^{t}(x, \nu)=\mathbf{c}\left(\tilde{\nu}-(1-t) J x-t \kappa_{\Phi}(x)\right)$. Let us see that $\sigma^{t}$ is a transversally elliptic symbol with support $\{(0,0)\}$ in $\mathrm{T}_{K}^{*} W$. Indeed if $\tilde{\nu}=(1-t) J x+t \kappa_{\Phi}(x)$ and is orthogonal to $\mathfrak{k} \cdot x$, we obtain

$$
0=\operatorname{Re}\left(\mathrm{h}\left(\tilde{\nu}, \kappa_{\Phi}(x)\right)\right)=2(1-t)\|\Phi(x)\|^{2}+t\left\|\kappa_{\Phi}(x)\right\|^{2},
$$


and this implies $x=0$. We obtain our first claim since the equivariant index of $\operatorname{At}(\bar{W})$ is $\operatorname{Sym}\left(W^{*}\right)$ (see Proposition 6.15).

Let us now prove that $\left[\operatorname{Sym}\left(W^{*}\right)\right]^{K}=\mathbb{C}$. If $P \in \operatorname{Sym}\left(W^{*}\right)$ is $K$-invariant, $P$ is constant on the trajectory of the vector field $\kappa_{\Phi}$ which is tangent to the $K$-orbits. So the value of $P$ coincide with the value of $P$ on the unique stationary point of $\kappa_{\Phi}$. So $P(x)=P(0)$ and $P$ is constant.

Remark 12.11 In the proof above we have checked that the transversally elliptic symbols $\sigma(W, \Phi)$ and $\operatorname{At}(\bar{W})$ define the same class in $\mathbf{K}_{K}^{0}\left(\mathrm{~T}_{K}^{*} W\right)$. If $\mathbb{T}$ is the 1-dimension center of $U(V)$, the same proof shows that $\sigma(W, \Phi)$ and $\operatorname{At}(\bar{W})$ define the same class in $\mathbf{K}_{K \times \mathbb{T}}^{0}\left(\mathrm{~T}_{\mathbb{W}}^{*} W\right)$

We now consider the general case where 0 is a quasi-regular value, and we study the character $R R_{K}\left(M, \mathcal{F}, \Phi^{-1}(0), \Phi\right)$ for any vector bundle $\mathcal{F} \rightarrow M$.

Let $\mathfrak{s}$ be the subalgebra of $\mathfrak{k}$ such that $Z:=\Phi^{-1}(0)$ is contained in the submanifold $M_{(\mathfrak{s})}$. Let $M_{\mathfrak{s}}=\left\{m \in M, \mathfrak{k}_{m}=\mathfrak{s}\right\}$. Let $H$ be the normalizer subgroup of $\mathfrak{s}$ in $K$. Thus $M_{(\mathfrak{s})}=K \times_{H} M_{\mathfrak{s}}$ and $Z=K \times_{H} Z_{\mathfrak{s}}$ where $Z_{\mathfrak{s}}:=\Phi^{-1}(0) \cap M_{\mathfrak{s}}$.

We write a $H$-invariant decomposition $\mathfrak{k}=\mathfrak{h} \oplus \mathfrak{q}$, with corresponding decomposition $\mathfrak{k}^{*}=\mathfrak{h}^{*} \oplus \mathfrak{q}^{*}$, and write $\Phi=\Phi_{\mathfrak{h}} \oplus \Phi_{\mathfrak{q}}$. Here $\Phi_{\mathfrak{h}}: M \rightarrow \mathfrak{h}^{*}$ is the moment map relative to the $H$-action on $M$, and the map $\Phi_{\mathfrak{q}}: M \rightarrow \mathfrak{q}^{*}$ is $H$-equivariant. We start with the basic lemma.

Lemma 12.12 The map $\Phi_{\mathfrak{q}}$ is equal to zero on $M_{\mathfrak{s}}$.

Proof. Let $m \in M_{\mathfrak{s}}$. Since $\mathfrak{k}_{m}=\mathfrak{s}$, the term $\Phi(m) \in \mathfrak{k}^{*} \simeq \mathfrak{k}$ satisfies $[\mathfrak{s}, \Phi(m)]=0$ and a fortiori $\Phi(m) \in \mathfrak{h}=N_{\mathfrak{k}}(\mathfrak{s})$.

Thus the restriction $\phi$ of $\Phi$ to $M_{\mathfrak{s}}$ takes values in $\mathfrak{h}^{*}$ : the manifold $M_{\mathfrak{s}}$ is a $H$-Hamiltonian manifold, with moment map $\phi$. Let $M_{\mathfrak{s}}^{0}$ be the union of connected components of $M_{\mathfrak{s}}$ that intersect $\phi^{-1}(0)$. Since $S$ acts trivially on $M_{\mathfrak{s}}^{0}$, the map $\phi$ take value in $(\mathfrak{h} / \mathfrak{s})^{*}$ on $M_{\mathfrak{s}}^{0}$. Since the group $H / S$ acts locally freely on $M_{\mathfrak{s}}^{0}$, the map $\phi: M_{\mathfrak{s}}^{0} \rightarrow(\mathfrak{h} / \mathfrak{s})^{*}$ is a submersion. In particular, $Z_{\mathfrak{s}}=$ $\phi^{-1}(0) \cap M_{\mathfrak{s}}^{0}$ is a compact submanifold, so $Z=K \times_{H} Z_{\mathfrak{s}}$ is a submanifold of $M$, as asserted previously, and the reduced space

$$
M_{0}:=\Phi^{-1}(0) / K=\phi^{-1}(0) \cap M_{\mathfrak{s}}^{0} /(H / S)
$$

is a compact symplectic orbifold. 
Let $\mathcal{N} \rightarrow Z$ be the symplectic normal bundle of the submanifold $Z$ in $M:$ for $x \in Z$,

$$
\left.\mathcal{N}\right|_{x}=\left(\mathrm{T}_{x} Z\right)^{\perp} /\left(\mathrm{T}_{x} Z\right)^{\perp} \cap \mathrm{T}_{x} Z .
$$

Here we have denoted by $\left(\mathrm{T}_{x} Z\right)^{\perp}$ the orthogonal with respect to the symplectic form.

We can equip $\mathcal{N}$ with an $H$-invariant Hermitian structure $\mathrm{h}$ such that the symplectic structure on the fibers of $\mathcal{N} \rightarrow Z$ is equal to $-\operatorname{Im}(\mathrm{h})$.

The subalgebra $\mathfrak{s}$ acts fiberwise on the complex vector bundle $\left.\mathcal{N}\right|_{Z_{\mathfrak{s}}}$. We consider the action of $\mathfrak{s}$ on the fibers of the complex bundle $\operatorname{Sym}\left(\mathcal{N}^{*} \mid Z_{\mathfrak{s}}\right)$. We will use the following.

Lemma 12.13 The subbundle $\left[\operatorname{Sym}\left(\left.\mathcal{N}^{*}\right|_{Z_{\mathfrak{s}}}\right)\right]^{\mathfrak{s}}$ is reduced to the trivial bundle $[\mathbb{C}] \rightarrow Z_{\mathfrak{s}}$.

Proof. Let $x \in Z_{\mathfrak{s}}$. The vector space $\mathfrak{k} \cdot x \subset \mathrm{T}_{x} M$ is totally isotropic, since $\Omega_{x}(X \cdot x, Y \cdot x)=\langle\Phi(x),[X, Y]\rangle=0$, hence we can consider the vector space $E_{x}:=(\mathfrak{k} \cdot x)^{\perp} / \mathfrak{k} \cdot x$ that is equipped with a $K_{x}$-equivariant symplectic structure $\Omega_{x}$ : we denote by $\Phi_{E_{x}}: E_{x} \rightarrow \mathfrak{s}^{*}$ the corresponding moment map. A local model for a symplectic neighborhood of $K x$ is $U:=K \times_{K_{x}}\left(\left(\mathfrak{k}_{x} / \mathfrak{s}\right)^{*} \times\right.$ $\left.E_{x}\right)$ where the moment map is $\Phi_{x}[k ; \xi, v]=k\left(\xi+\Phi_{E_{x}}(v)\right)$. Our hypothesis that $\Phi^{-1}(0)$ is contained in $M_{(\mathfrak{s})}$ implies that $\Phi_{E_{x}}^{-1}(0)=\left(E_{x}\right)^{\mathfrak{s}}$. We get then the decompositions

$$
\begin{aligned}
\mathrm{T}_{x} Z & =(\mathfrak{k} / \mathfrak{s}) \cdot x \oplus\left(E_{x}\right)^{\mathfrak{s}}, \\
\mathrm{T}_{x} M & =(\mathfrak{k} / \mathfrak{s}) \cdot x \oplus(\mathfrak{k} / \mathfrak{s})^{*} \oplus E_{x} .
\end{aligned}
$$

Let $S$ be the connected component of $K_{x}$. We see then that $\left.\mathcal{N}\right|_{x}$ is equal to the symplectic orthogonal of $\left(E_{x}\right)^{\mathfrak{s}}$ in $E_{x}$. Hence $\left.\mathcal{N}\right|_{x}$ is a symplectic vector space with a Hamiltonian action of the compact group $S$ such that $\Phi_{\left.\mathcal{N}\right|_{x}}^{-1}(0)=\left.\Phi_{E_{x}}^{-1}(0) \cap \mathcal{N}\right|_{x}=0$. As we have seen before, it implies that $\left[\operatorname{Sym}\left(\left.\mathcal{N}^{*}\right|_{x}\right)\right]^{\mathfrak{s}}=\mathbb{C}$.

Definition 12.14 If $\mathcal{F} \rightarrow M$ is a $K$ - equivariant complex vector bundle, we define on $M_{0}$ the (finite dimensional) orbi-bundle

$$
\mathcal{F}_{0}:=\left[\left.\mathcal{F}\right|_{Z_{\mathfrak{s}}} \otimes \operatorname{Sym}\left(\left.\mathcal{N}^{*}\right|_{Z_{\mathfrak{s}}}\right)\right]^{\mathfrak{s}} /(H / S) .
$$

If $\mathfrak{s}$ acts trivially on the fibers of $\left.\mathcal{F}\right|_{Z_{\mathfrak{s}}}$, the bundle $\mathcal{F}_{0}$ is equal to $\left.\mathcal{F}\right|_{Z_{\mathfrak{s}}} /(H / S)$.

The aim of this subsection is the following theorem. 
Theorem 12.15 Assume that $\Phi^{-1}(0) \subset M_{(\mathfrak{s})}$. For any $K$-equivariant complex vector bundle $\mathcal{F} \rightarrow M$, we have

$$
\left[R R_{K}\left(M, \mathcal{F}, \Phi^{-1}(0), \Phi\right)\right]^{K}=R R\left(M_{0}, \mathcal{F}_{0}\right) .
$$

The proof of this theorem will require two steps, inducing and restricting, that are considered in the next subsections.

\section{Inducing from $H$ to $K$.}

Let us show that there is a $K$-invariant neighborhood $N$ of the compact submanifold $Z_{\mathfrak{s}}:=\Phi^{-1}(0) \cap M_{\mathfrak{s}}$ of $M$ of the form $N=K \times_{H}\left(N^{\prime} \times B_{\mathfrak{q}^{*}}\right)$, where $B_{\mathfrak{q}^{*}}$ is a small $H$-invariant ball in $\mathfrak{q}^{*}$, and $N^{\prime}$ a $H$-invariant symplectic submanifold of $M$.

Let $\mathcal{V} \subset M$ be a small $K$-invariant tubular neighborhood of the stratum $M_{(\mathfrak{s})}$ with fibration $p: \mathcal{V} \rightarrow M_{(\mathfrak{s})}$. Let $V=p^{-1}\left(M_{\mathfrak{s}}\right)$. Thus $V$ is a $H$-invariant submanifold of $M$ containing $M_{\mathfrak{s}}$, fibered over $M_{\mathfrak{s}}$ and $\mathcal{V}=K \times_{H} V$ is an open subset of $M$. We consider the map $\Phi_{\mathfrak{q}}: V \rightarrow \mathfrak{q}^{*}$.

Lemma 12.16 Let $z \in Z_{\mathfrak{s}}$ :

(a) the differential $\mathrm{T}_{z} \Phi_{\mathfrak{q}}: \mathrm{T}_{z} V \rightarrow \mathfrak{q}^{*}$ is surjective,

(b) $\operatorname{ker}\left(\mathrm{T}_{z} \Phi_{\mathfrak{q}}\right) \subset \mathrm{T}_{z} V$ is a symplectic subspace of $\mathrm{T}_{z} M$.

Proof. For $z \in Z_{\mathfrak{s}}$, we have $\mathrm{T}_{z} M=\mathfrak{q} \cdot z \oplus \mathrm{T}_{z} V$ with $\mathfrak{q} \cdot z \simeq \mathfrak{q}$ since $\mathfrak{k}_{z}=\mathfrak{s}$. The subspace $\mathfrak{q} \cdot z$ is totally isotropic since $\Phi(z)=0$, and by definition the subspace $\operatorname{ker}\left(\mathrm{T}_{z} \Phi_{\mathfrak{q}}\right) \subset \mathrm{T}_{z} V$ is equal to $(\mathfrak{q} \cdot z)^{\perp} \cap \mathrm{T}_{z} V$. So we have $(\mathfrak{q} \cdot z)^{\perp}=\mathfrak{q} \cdot z \oplus \operatorname{ker}\left(\mathrm{T}_{z} \Phi_{\mathfrak{q}}\right)$. This implies that $\operatorname{ker}\left(\mathrm{T}_{z} \Phi_{\mathfrak{q}}\right) \subset \mathrm{T}_{z} V$ is a symplectic subspace of $\mathrm{T}_{z} M$ of dimension equal to $\operatorname{dim}\left(\mathrm{T}_{z} V\right)-\operatorname{dim}(\mathfrak{q})$ : the surjectivity of $\mathrm{T}_{z} \Phi_{\mathfrak{q}}: \mathrm{T}_{z} V \rightarrow \mathfrak{q}^{*}$ follows.

Let $\mathcal{X}$ be a small $H$-invariant neighborhood of $Z_{\mathfrak{s}}$ in $M_{\mathfrak{s}}$, and let $V_{\mathcal{X}}$ be a small neighborhood of $\mathcal{X}$ in $p^{-1}(\mathcal{X}) \subset V$ such that for any $z \in V_{\mathcal{X}}$ the statements $(a)$ and $(b)$ hold.

Consider $N^{\prime}:=\left\{z \in V_{\mathcal{X}} ; \Phi_{\mathfrak{q}}(z)=0\right\}$. Then $N^{\prime}$ is a $H$-Hamiltonian submanifold of $V_{\mathcal{X}}$ containing $\mathcal{X}$, with symplectic form $\Omega^{\prime}:=\left.\Omega\right|_{N^{\prime}}$ and moment map $\Phi^{\prime}: N^{\prime} \rightarrow \mathfrak{h}^{*}$ equal to the restriction of $\Phi$ to $N^{\prime}$. If $\mathcal{X}$ is small enough, there exists an invariant neighborhood $U^{\prime}$ of $\mathcal{X}$ in $V_{\mathcal{X}}$ and a diffeomorphism $j: N^{\prime} \times B_{\mathfrak{q}^{*}} \rightarrow U^{\prime}$ such that $\Phi \circ j=\Phi^{\prime}\left(n^{\prime}\right)+\xi$, for any $\left(n^{\prime}, \xi\right) \in N^{\prime} \times B_{\mathfrak{q}^{*}}$. 
Finally we have proved that a neighborhood of $Z_{\mathfrak{s}}$ in $M$ is diffeomorphic to $N=K \times_{H}\left(N^{\prime} \times B_{\mathfrak{q}^{*}}\right)$. Here $\left(N^{\prime}, \Omega^{\prime}, \Phi^{\prime}\right)$ is a $H$-Hamiltonian submanifold of $(M, \Omega, \Phi)$ and the moment map $\Phi_{N}: N \rightarrow \mathfrak{k}^{*}$ is defined by the relation

$$
\Phi_{N}\left(\left[k ; n^{\prime}, \xi\right]\right)=k \cdot\left(\Phi^{\prime}\left(n^{\prime}\right)+\xi\right) .
$$

We return to the study of $R R_{K}\left(M, \mathcal{F}, \Phi^{-1}(0), \Phi\right)$ under the hypothesis that $\Phi^{-1}(0)$ is contained in $M_{(\mathfrak{s})}$. We may do the computation in the neighborhood $N$ of $\Phi^{-1}(0)$. Note that $\Phi^{-1}(0) \subset M_{(\mathfrak{s})}$ implies that $Z^{\prime}:=\left(\Phi^{\prime}\right)^{-1}(0)$ is equal to $Z_{\mathfrak{s}}$. Recall that $R R_{K}\left(N, \mathcal{F}, \Phi^{-1}(0), \Phi\right)=\mathcal{Q}_{K}\left(N, \bigwedge_{J} \mathrm{~T} N \otimes\right.$ $\left.\mathcal{F}, \Phi^{-1}(0), \Phi\right)$. Here we have chosen $J_{N}$, an almost complex structure that is compatible with the symplectic form $\Omega$. We may then further deform the almost complex structure $J_{N}$ and we will keep the same index.

Let $J_{N^{\prime}}$ be an almost complex structure on $N^{\prime}$ that is compatible with $\Omega^{\prime}$. If we restrict the Clifford bundle $\mathcal{E}:=\bigwedge_{J_{N}} \mathrm{~T} N$ to the submanifold $N^{\prime}$, we get

$$
\left.\mathcal{E}\right|_{N^{\prime}}=S_{\mathfrak{q}} \otimes \mathcal{E}^{\prime}
$$

where $S_{\mathfrak{q}}=\bigwedge_{\mathbb{C}} \mathfrak{q}_{\mathbb{C}}$. We need to see that $\mathcal{E}^{\prime}=\bigwedge_{J_{N^{\prime}}} \mathrm{T} N^{\prime}$.

At each point $y$ of $N^{\prime}$, the tangent bundle $\mathrm{T}_{y} N$ admits a canonical identification with $\mathfrak{q} \oplus \mathfrak{q}^{*} \oplus \mathrm{T}_{y} N^{\prime}$. Through the identification $X \oplus \xi \in$ $\mathfrak{q} \oplus \mathfrak{q}^{*} \mapsto X \oplus i \tilde{\xi} \in \mathfrak{q} \mathbb{C}$, the vector space $\mathfrak{q} \oplus \mathfrak{q}^{*}$ inherits a complex structure $J_{\mathfrak{q}}$ and a Hermitian form. Take a Riemannian metric $g$ on $\mathrm{T} N$, orthogonal direct sum of the metric $\Omega^{\prime}\left(\cdot, J_{N^{\prime}} \cdot\right)$ and of the underlying Riemannian metric of $\mathfrak{q}_{\mathbb{C}}$. The symplectic form $\Omega_{y}$ can be written $g\left(\cdot, A_{y} \cdot\right)$ where $A_{y}$ is a non degenerate antisymmetric matrix. Let $J_{y}=\frac{A_{y}}{\sqrt{-A_{y} A_{y}^{*}}}$ be the almost complex structure determined by $A_{y}$. When $y \in Z_{\mathfrak{s}}$, $A_{y}$ is equal to $J_{N^{\prime}} \oplus J_{\mathfrak{q}}$. As $Z_{\mathfrak{s}}$ is compact, we see that if $N^{\prime}$ is a sufficiently small neighborhhod, $J_{y}$ is homotopic to $J_{N^{\prime}} \oplus J_{\mathfrak{q}}$, and we obtain our result.

Proposition 11.1 tells us then that

$$
\left[R R_{K}\left(M, \mathcal{F}, \Phi^{-1}(0), \Phi\right)\right]^{K}=\left[R R_{H}\left(N^{\prime}, \mathcal{F}, Z^{\prime}, \Phi^{\prime}\right)\right]^{H} .
$$

Here $Z^{\prime}=Z_{\mathfrak{s}}$, and we have still denoted by $\mathcal{F}$ the restriction of $\mathcal{F}$ to $N^{\prime}$.

\section{Localization on $M_{\mathfrak{s}}$.}

We now analyze $\left[R R_{H}\left(N^{\prime}, \mathcal{F}, Z^{\prime}, \Phi^{\prime}\right)\right]^{H}$, where $Z^{\prime}=Z_{\mathfrak{s}}$.

Let us summarize the situation. We consider a $H$-equivariant decomposition $\mathfrak{h}=\mathfrak{s} \oplus \mathfrak{r}$. Here $\mathcal{X}$ is a small neighborhood of $Z_{\mathfrak{s}}$ in $M_{\mathfrak{s}}, N^{\prime}$ is a $H$-Hamiltonian manifold, with symplectic structure $\Omega^{\prime}$ and moment map 
$\Phi^{\prime}: N^{\prime} \rightarrow \mathfrak{h}^{*}$. We have $N_{\mathfrak{s}}^{\prime}=\mathcal{X}$. The restriction $\Phi_{\mathcal{X}}$ of $\Phi^{\prime}$ to $\mathcal{X}$ takes its values in $\mathfrak{r}^{*}$, and the zero set $Z^{\prime}$ of the vector field $\kappa_{\Phi^{\prime}}$ is the compact submanifold $\Phi_{\mathcal{X}}^{-1}(0)=Z_{\mathfrak{s}}$ of $\mathcal{X}$.

We denote by $\kappa_{\mathcal{X}}$ the Kirwan vector field on $\mathcal{X} \subset M_{\mathfrak{s}}$ associated to $\Phi_{\mathcal{X}}$.

As we work on a neighborhood of $Z^{\prime}$, we may assume that $N^{\prime}$ is diffeomorphic to $\mathcal{N}^{\prime}$, the total space of the normal bundle of $\mathcal{X}$ in $N^{\prime}$. The restriction of $\Omega^{\prime}$ on $\mathcal{X}$ induces a symplectic structure on each fibers : $\left.\Omega_{x} \in \Lambda^{2} \mathcal{N}^{\prime}\right|_{x}$ for $x \in \mathcal{X}$.

We choose an $H$-invariant connection on $\mathcal{N}^{\prime}$. The subspace $\mathfrak{r} \cdot w$ of $\mathrm{T}_{w} \mathcal{W}$ projects injectively on $\mathfrak{r} \cdot x$, and keep the same dimension $\operatorname{dim} \mathfrak{r}$. Thus we can assume that the horizontal space contains $\mathfrak{r} \cdot w$.

If $\eta \in \mathrm{T}_{w} \mathcal{N}^{\prime}$, we denote $\eta^{\text {vert }}$ its vertical component. Let $\lambda$ be the $H$ invariant one-form on $\mathcal{N}^{\prime}$ such that, at a point $w=(x, v) \in \mathcal{N}^{\prime}, \lambda_{w}(\eta)=$ $\Omega_{x}\left(v, \eta^{\text {vert }}\right)$. From the symplectic embedding theorem, we may assume that $\Omega^{\prime}=d \lambda+p^{*} \Omega_{\mathcal{X}}$. Here $p$ is the projection $\mathcal{N}^{\prime} \rightarrow \mathcal{X}$.

We can equip the vector bundle $\mathcal{N}^{\prime}$ with a $H$-equivariant Hermitian structure such that the section $x \in \mathcal{X} \mapsto \Omega_{x}$ is minus the imaginary part of the Hermitian metric. Thus the complex structure $J$ on the fiber $\left.\mathcal{N}^{\prime}\right|_{x}$ is compatible with $\Omega_{x}$. Let us choose a $H$-invariant metric on $\mathcal{X}$, and let $J_{\mathcal{X}}$ be the corresponding almost complex structure on $\mathcal{X}$ compatible with $\Omega_{\mathcal{X}}$. Thanks to the connection, we can consider on the manifold $\mathcal{N}^{\prime}$ a $H$-invariant metric orthogonal direct sum of the metric on $\mathcal{X}$ (its horizontal lift) and the Hermitian metric on $\mathcal{N}^{\prime}$, let $J_{\mathcal{N}^{\prime}}$ be the corresponding compatible almost complex structure associated to $\Omega^{\prime}$ on $\mathcal{N}^{\prime}$. We consider also with the help of the connection $J^{\prime}=J \oplus J_{\mathcal{X}}$. On $\mathcal{X}$, we have $J^{\prime}=J_{\mathcal{N}^{\prime}}$. Thus $J_{\mathcal{N}^{\prime}}$ is a deformation of $J^{\prime}$ and $R R_{H}\left(N^{\prime}, \mathcal{F}, Z^{\prime}, \Phi^{\prime}\right)$ can be computed using the direct sum $J^{\prime}$.

Remark that we have the additional action of the group $U(1)$ acting by homotheties $v \mapsto e^{i \theta} v$ on the fibers of the Hermitian bundle $\mathcal{N}^{\prime} \rightarrow \mathcal{X}$ and commuting with the $H$-action, with infinitesimal generator $J$. Thus we have a morphism $\mathrm{At}_{J}: \mathbf{K}_{H}^{0}\left(\mathrm{~T}_{H}^{*} \mathcal{X}\right) \longrightarrow \mathbf{K}_{H}^{0}\left(\mathrm{~T}_{H}^{*} \mathcal{N}^{\prime}\right)$ defined by Equation (6.18).

We compute now the moment map $\Phi^{\prime}$ associated to the action of $H$ on $\left(\mathcal{N}^{\prime}, \Omega^{\prime}\right)$. We write $\Phi^{\prime}=\Phi_{\mathfrak{s}} \oplus \Phi_{\mathfrak{r}}$. We obtain for $w=(x, v) \in \mathcal{N}^{\prime}$ :

- $\left\langle\Phi_{\mathfrak{s}}(x, v), X\right\rangle=\frac{1}{2} \Omega_{x}(X v, v)$ if $X \in \mathfrak{s}$,

- $\Phi_{\mathfrak{r}}(x, v)=\Phi_{\mathcal{X}}(x)$.

Let $\mathcal{N} \rightarrow Z$ be the symplectic normal bundle of the submanifold $Z$ in $M$, and let $\left.\mathcal{N}\right|_{Z_{\mathfrak{s}}}$ be its restriction on $Z_{\mathfrak{s}}$. We will need the following 
Lemma 12.17 • The restriction of symplectic vector bundle $\mathcal{N}^{\prime} \rightarrow \mathcal{X}$ to $Z_{\mathfrak{s}}$ is equal to $\left.\mathcal{N}\right|_{Z_{\mathfrak{s}}}$.

- The subbundle $\left[\operatorname{Sym}\left(\left(\mathcal{N}^{\prime}\right)^{*}\right)\right]^{\mathfrak{s}}$ is reduced to the trivial bundle $[\mathbb{C}] \rightarrow \mathcal{X}$.

Proof. Let $x \in Z_{\mathfrak{s}}$. Let $\mathfrak{q}_{x}^{*}$ be a subspace of $\left(\mathrm{T}_{x} N^{\prime}\right)^{\perp} \subset \mathrm{T}_{x} M$ that is in bijection with $\mathfrak{q}^{*}$ through the tangent map $\mathrm{T}_{x} \Phi_{\mathfrak{q}}$. Let $(\mathfrak{h} / \mathfrak{s})_{x}^{*}$ be a subspace of $\mathrm{T}_{x} \mathcal{X}$ that is in bijection with $(\mathfrak{h} / \mathfrak{s})^{*}$ through the tangent map $\mathrm{T}_{x} \Phi_{\mathfrak{h}}$. We have the decompositions

$$
\begin{aligned}
\mathrm{T}_{x} M & =\left(\mathfrak{q} \cdot x \oplus \mathfrak{q}_{x}^{*}\right) \stackrel{\perp}{\oplus} \mathrm{T}_{x} N^{\prime} \\
\mathrm{T}_{x} N^{\prime} & =\left.\mathrm{T}_{x} \mathcal{X} \oplus \mathcal{N}^{\prime}\right|_{x} \\
\mathrm{~T}_{x} \mathcal{X} & =(\mathfrak{h} / \mathfrak{s})_{x}^{*} \oplus \mathrm{T}_{x} Z_{\mathfrak{s}} .
\end{aligned}
$$

that gives $\mathrm{T}_{x} M=\left.\left(\mathfrak{q} \cdot x \oplus \mathfrak{q}_{x}^{*}\right) \stackrel{\perp}{\oplus}\left((\mathfrak{h} / \mathfrak{s})_{x}^{*} \oplus \mathrm{T}_{x} Z_{\mathfrak{s}}\right) \stackrel{\perp}{\oplus} \mathcal{N}^{\prime}\right|_{x}$. Now, if we use that $\mathrm{T}_{x} Z=\mathfrak{q} \cdot x \oplus \mathrm{T}_{x} Z_{\mathfrak{s}}$, we see that $\left(\mathrm{T}_{x} Z\right)^{\perp}=\left.\left(\mathrm{T}_{x} Z\right)^{\perp} \cap \mathrm{T}_{x} Z \stackrel{\perp}{\oplus} \mathcal{N}^{\prime}\right|_{x}$, and then $\left.\mathcal{N}\right|_{x}=\left.\mathcal{N}^{\prime}\right|_{x}$. The first point is proved.

The restriction of the bundle $\left[\operatorname{Sym}\left(\left(\mathcal{N}^{\prime}\right)^{*}\right)\right]^{\mathfrak{s}}$ to $Z_{\mathfrak{s}}$ is equal to $\left[\operatorname{Sym}\left(\left.\mathcal{N}\right|_{Z_{\mathfrak{s}}} ^{*}\right)\right]^{\mathfrak{s}}$ which is the trivial bundle $[\mathbb{C}] \rightarrow Z_{\mathfrak{s}}$. The second point follows.

We are in a very similar situation than Proposition $7.5:$ instead of $\beta$ acting fiberwise, we have $\mathfrak{s}$ (an $H$-stable ideal in $\mathfrak{h}$ ) acting fiberwise.

Let $w=(x, v) \in \mathcal{N}^{\prime}$, with $x \in \mathcal{X}$, and $\left.v \in \mathcal{N}^{\prime}\right|_{x}$. The horizontal lift of $\kappa_{\mathcal{X}}$ (still denoted by $\kappa_{\mathcal{X}}$ ) is the vector field $\Phi_{\mathfrak{r}}(w) \cdot w$. As in the proof of Proposition 12.9, we introduce the deformation $\kappa^{t}(w)=-\Phi_{\mathfrak{r}}(w) \cdot w-$ $t \Phi_{\mathfrak{s}}(w) \cdot w+(1-t) J w$ and

$$
\sigma^{t}(w, \nu)=\mathbf{c}_{w}\left(\tilde{\nu}-\kappa^{t}(w)\right), \quad(w, \nu) \in \mathrm{T}^{*} \mathcal{N}^{\prime}
$$

acting on $\bigwedge_{J^{\prime}} \mathrm{T}^{\prime} \otimes \mathcal{F}$. Note that the vector $\Phi_{\mathfrak{r}}(w) \cdot w$ is horizontal, while $\Phi_{\mathfrak{s}}(w) \cdot w$ and $J w$ are verticals.

By definition, the character $R R_{H}\left(N^{\prime}, \mathcal{F}, Z^{\prime}, \Phi^{\prime}\right)$ is equal to the equivariant index of $\sigma^{1} \otimes \mathcal{F}$. Let us prove that the characteristic set of $\sigma^{t}$ intersected with $\mathrm{T}_{H}^{*} \mathcal{N}^{\prime}$ stays equal to $Z_{\mathfrak{s}}$. Consider $\eta=\kappa^{t}(w)$ and orthogonal to $\mathfrak{h} \cdot w$. Thus we obtain $\left\langle\Phi_{\mathfrak{r}}(w) \cdot w+t \Phi_{\mathfrak{s}}(w) \cdot w-(1-t) J w, \Phi_{\mathfrak{r}}(w) \cdot w\right\rangle=0$ and $\left\langle\Phi_{\mathfrak{r}}(w) \cdot w+t \Phi_{\mathfrak{s}}(w) \cdot w-(1-t) J w, \Phi_{\mathfrak{s}}(w) \cdot w\right\rangle=0$. Thus considering vertical and orthogonal components, we obtain $\Phi_{\mathfrak{r}}(w)=0$. Then we have

$$
\begin{aligned}
0 & =\left\langle t \Phi_{\mathfrak{s}}(w) \cdot w-(1-t) J w, \Phi_{\mathfrak{s}}(w) \cdot w\right\rangle \\
& =t\left\|\Phi_{\mathfrak{s}}(w) \cdot w\right\|^{2}+2(1-t)\left\|\Phi_{\mathfrak{s}}(w)\right\|^{2}
\end{aligned}
$$


as $\left\langle J w, \Phi_{\mathfrak{s}}(w) \cdot w\right\rangle=-2\left\|\Phi_{\mathfrak{s}}(w)\right\|^{2}$.

Thus we have proved that if $\kappa^{t}(w)$ is orthogonal to $\mathfrak{h} \cdot w$, then $\Phi^{\prime}(w)=0$ : this implies $w=(x, 0)$ with $x \in Z_{\mathfrak{s}}$.

We know then that $R R_{H}\left(N^{\prime}, \mathcal{F}, Z^{\prime}, \Phi^{\prime}\right)$ is equal to the equivariant index of the transversally elliptic symbol $\sigma^{0} \otimes \mathcal{F}$. Since the symbol $\sigma^{0}$ is equal to $\operatorname{At}_{J}\left(\sigma\left(\mathcal{X}, Z_{\mathfrak{s}}, \Phi_{\mathcal{X}}\right)\right)$, we have then by Equation (6.19)

$$
\left[R R_{H}\left(N^{\prime}, \mathcal{F}, Z^{\prime}, \Phi^{\prime}\right)\right]^{H}=\left[R R_{H}\left(\mathcal{X}, \mathcal{F} \otimes \operatorname{Sym}\left(\left(\mathcal{N}^{\prime}\right)^{*}\right), Z_{\mathfrak{s}}, \Phi_{\mathcal{X}}\right)\right]^{H} .
$$

We obtain

$$
\begin{aligned}
& {\left[R R_{H}\left(\mathcal{X}, \mathcal{F} \otimes \operatorname{Sym}\left(\left(\mathcal{N}^{\prime}\right)^{*}\right), Z_{\mathfrak{s}}, \Phi_{\mathcal{X}}\right)\right]^{H}} \\
& \quad=\left[R R_{H / S}\left(\mathcal{X},\left[\mathcal{F} \otimes \operatorname{Sym}\left(\left(\mathcal{N}^{\prime}\right)^{*}\right)\right]^{\mathfrak{s}}, Z_{\mathfrak{s}}, \Phi_{\mathcal{X}}\right)\right]^{H / S}, \\
& \quad=R R\left(M_{0}, \mathcal{F}_{0}\right)
\end{aligned}
$$

where $\mathcal{F}_{0}:=\left[\left.\mathcal{F}\right|_{Z_{\mathfrak{s}}} \otimes \operatorname{Sym}\left(\left.\mathcal{N}^{*}\right|_{Z_{\mathfrak{s}}}\right)\right]^{\mathfrak{s}} /(H / S)$. In (1), we used the regular case considered in Proposition 12.5.

\subsection{Riemann-Roch number on symplectic reduction}

Let $(M, \Omega, \Phi)$ be a Hamiltonian $K$-manifold not necessarily compact. Suppose that $a$ is a quasi-regular value of $\Phi$ such that the fiber $Z:=\Phi^{-1}(a)$ is a compact submanifold. The quotient $M_{a}:=\Phi^{-1}(a) / K_{a}$ is a compact symplectic orbifold.

Let $\mathfrak{s} \subset \mathfrak{k}_{a}$ be a subalgebra such that $\Phi^{-1}(K a)$ is contained in the submanifold $M_{(\mathfrak{s})}$. We denote by $S$ the connected subgroup of $K_{a}$ with Lie algebra $\mathfrak{s}$, and $H$ the normalizer subgroup of $\mathfrak{s}$ in $\mathfrak{k}_{a}$. The orbifold $M_{a}=\Phi^{-1}(a) / K_{a}$ is also the quotient of the submanifold $Z_{\mathfrak{s}}:=\Phi^{-1}(a) \cap M_{\mathfrak{s}}$ by the group $H / S$.

We consider now the case of an equivariant vector bundle $\mathcal{F} \rightarrow M$ and a character $\chi_{\lambda}: K_{a} \rightarrow \mathrm{U}(1):$ here $\lambda=\frac{1}{i} d \chi_{\lambda}$ is a weight, and we denote $\mathbb{C}_{\lambda}$ the 1-dimensional representation of $K_{a}$ defined by $\chi_{\lambda}$.

We suppose that the action of $S$ on the fibers of the bundle $\left.\mathcal{F}\right|_{Z_{\mathfrak{s}}}$ is $t \cdot v=\chi_{\lambda}(t) v$. Then $S$ acts trivially on $\left.\mathcal{F}\right|_{Z_{\mathfrak{s}}} \otimes \mathbb{C}_{-\lambda}$ and we may form the orbibundle

$$
\mathcal{F}_{a, \lambda}:=\left(\left.\mathcal{F}\right|_{Z_{\mathfrak{s}}} \otimes \mathbb{C}_{-\lambda}\right) /(H / S)
$$

on $M_{a}$. The aim of this section is to show that the Riemann-Roch number $R R\left(M_{a}, \mathcal{F}_{a, \lambda}\right)$ can be computed by a localization procedure.

Let $(K a)^{-}$be the coadjoint orbit $K a$ with the opposite symplectic structure. Let $\left[\mathbb{C}_{-\lambda}\right]=K \times_{K_{a}} \mathbb{C}_{-\lambda}$ be the line bundle on $(K a)^{-}$. We consider 
the symplectic manifold $M \times(K a)^{-}$with the complex bundle $\mathcal{F} \otimes\left[\mathbb{C}_{-\lambda}\right]$ on it. The corresponding moment map $\Phi_{a}: M \times(K a)^{-} \rightarrow \mathfrak{k}^{*}$ is $\Phi_{a}(m, \xi)=$ $\Phi(m)-\xi$. As the set $\Phi_{a}^{-1}(0)$ is compact, we may define the localized Riemann Roch character

$$
R R_{K}\left(M \times(K a)^{-}, \mathcal{F} \otimes\left[\mathbb{C}_{-\lambda}\right], \Phi_{a}^{-1}(0), \Phi_{a}\right) \in \hat{R}(K) .
$$

The main result of this section is the

Theorem 12.18 If a is a quasi-regular value of $\Phi$ we have

$$
\left[R R_{K}\left(M \times(K a)^{-}, \mathcal{F} \otimes\left[\mathbb{C}_{-\lambda}\right], \Phi_{a}^{-1}(0), \Phi_{a}\right)\right]^{K}=R R\left(M_{a}, \mathcal{F}_{a, \lambda}\right) .
$$

Proof. Let $B \subset \mathfrak{k}_{a}^{*}$ be a small $K_{a}$-invariant ball around $a$, and consider the slice $Y:=\Phi^{-1}(B)$ which is a symplectic submanifold of $M$ : the action of $K_{a}$ on $\left(Y,\left.\Omega\right|_{Y}\right)$ is Hamiltonian with moment map $\Phi_{Y}:=\left.\Phi\right|_{Y}$.

The computation of the left hand side of (12.39) is done in the manifold $\tilde{M} \times(K a)^{-}$where $\tilde{M}=K \times_{K_{a}} Y$ is viewed as an open subset of $M$. Let $J_{Y}$ be a $K_{a}$-invariant almost complex structure on $Y$ compatible with the symplectic form $\left.\Omega\right|_{Y}$. Let $J_{\tilde{M}}$ be the $K$-invariant complex structure on $\tilde{M}$ that is equal to $J_{a} \oplus J_{Y}$ at the points $[1, y], y \in Y$. Notice that $J_{\tilde{M}}$ is compatible with the symplectic structure $\left.\Omega\right|_{\tilde{M}}$.

If we apply the induction formula of Proposition 11.3 to the manifold $\tilde{M} \times(K a)^{-}$equipped with the Clifford bundles $\mathcal{G}:=\bigwedge_{J} \mathrm{~T} M \otimes \mathcal{F}$ on $\tilde{M}$ and $\mathcal{R}=\bigwedge_{-J_{a}} \mathrm{~T} K a \otimes\left[\mathbb{C}_{-\lambda}\right]$ on $K a$, we get

- $\mathcal{G}_{d Y}=\left.\bigwedge_{J_{Y}} \mathrm{~T} Y \otimes \mathcal{F}\right|_{Y}$ for the corresponding Clifford bundle on $Y$,

- $R=\mathbb{C}_{-\lambda}$ for the corresponding $K_{a}$-module.

Hence we have

$$
\begin{aligned}
& {\left[R R_{K}\left(M \times(K a)^{-}, \mathcal{F} \otimes\left[\mathbb{C}_{-\lambda}\right], \Phi_{a}^{-1}(0), \Phi_{a}\right)\right]^{K}} \\
& \quad=\left[R R_{K_{a}}\left(Y, \mathcal{F}_{\lambda}, \phi^{-1}(0), \phi\right)\right]^{K_{a}} .
\end{aligned}
$$

where $\phi:=\Phi_{Y}-a$ is a moment map on the $K_{a}$-Hamiltonian manifold $\left(Y, \Omega_{Y}\right), 0$ is a quasi regular value of $\phi$ and $\mathcal{F}_{\lambda}=\left.\mathcal{F}\right|_{Y} \otimes \mathbb{C}_{-\lambda}$.

We are in the setting of Section 12.2.3. Since $\mathfrak{s}$ acts trivially on the fibers of the vector bundle $\left.\mathcal{F}_{\lambda}\right|_{Z_{\mathfrak{s}}}$, Theorem 12.15 says that

$$
\left[R R_{K_{a}}\left(Y, \mathcal{F}_{\lambda}, \phi^{-1}(0), \phi\right)\right]^{K_{a}}=R R\left(M_{a}, \mathcal{F}_{a, \lambda}\right)
$$

with $\mathcal{F}_{a, \lambda}=\left.\mathcal{F}_{\lambda}\right|_{Z_{\mathfrak{s}}} /(H / S)$. The proof is completed. 


\subsection{Multiplicities as Riemann-Roch numbers}

In this section, we give a proof of the $[Q, R]=0$ Theorem of MeinrenkenSjamaar.

Let $L$ be a $\Phi$-moment line bundle on the compact Hamiltonian $K$ manifold $(M, \Omega, \Phi)$. We fix a dominant weight $\mu$, and we consider the multiplicity $\mathrm{m}_{\mu}(L)=\left[R R_{K}(M, L) \otimes V_{\mu}^{*}\right]^{K}$. We consider elements $a \in \mathfrak{t}^{*}$ close enough to $\mu$, so $K_{a} \subset K_{\mu}$. Let $(K a)^{-}$be the coadjoint orbit equipped with the opposite symplectic structure and the line bundle $\left[\mathbb{C}_{-\mu}\right] \simeq K \times_{K_{a}} \mathbb{C}_{-\mu}$. We have $R R_{K}\left((K a)^{-},\left[\mathbb{C}_{-\mu}\right]\right)=V_{\mu}^{*}$, hence the shifting trick gives

$$
\mathrm{m}_{\mu}(L)=\left[R R_{K}\left(M \times(K a)^{-}, \mathbb{L}(a)\right)\right]^{K} .
$$

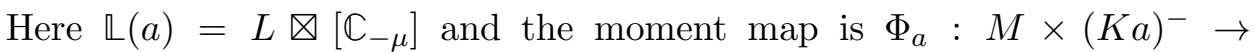
$\mathfrak{k}^{*},(m, \xi) \mapsto \Phi(m)-\xi$. We start with the

Lemma 12.19 There exist $\epsilon>0$ such that the line bundle $\mathbb{L}(a)$ is weakly $\Phi_{a}$-positive if $\|a-\mu\| \leq \epsilon$.

Proof. We consider the maps $\varphi_{a}: M \times K / T \rightarrow M \times(K a)^{-}$defined by $\varphi(m,[k])=(m, k a)$. The line bundle $\varphi_{a}^{*} \mathbb{L}(a)$ does not depends of $a$ and is denoted $\mathbb{L}^{\prime}$. Let $\Phi_{a}^{\prime}=\varphi_{a}^{*} \Phi_{a}$.

One checks that $\varphi_{a}\left(\left\{\kappa_{\Phi_{a}^{\prime}}=0\right\}\right)=\left\{\kappa_{\Phi_{a}}=0\right\}$, hence we can use Lemma 9.3 : the line bundle $\mathbb{L}(a)$ is weakly $\Phi_{a}$-positive if and only if the line bundle $\mathbb{L}^{\prime}$ is weakly $\Phi_{a}^{\prime}$-positive.

Now we see that for $a=\mu$, the line bundle $\mathbb{L}^{\prime}$ is a $\Phi_{\mu}^{\prime}$-moment line bundle. Thanks to Proposition 9.7, we know that $\mathbb{L}^{\prime}$ is weakly $\Phi_{a}^{\prime}$-positive if $a$ is close enough to $\mu$.

Now we take $a \in \Delta(M)$ such that $\|a-\mu\| \leq \epsilon$. Since $\mathbb{L}(a)$ is a weakly $\Phi_{a}$-positive line bundle on the Hamiltonian $K$-manifold $M \times(K a)^{-}$and $\Phi_{a}^{-1}(0) \neq \emptyset$, Theorem 10.7 says that

$$
\mathrm{m}_{\mu}(L)=\left[R R_{K}\left(M \times(K a)^{-}, \mathbb{L}(a), \Phi_{a}^{-1}(0), \Phi_{a}\right)\right]^{K} .
$$

We suppose furthermore that $a$ is a quasi-regular value of $\Phi$. Let $\mathfrak{s}$ be the subalgebra of $\mathfrak{k}_{a}$ such that $\Phi^{-1}(K a)$ is contained in $M_{(\mathfrak{s})}$ : we denote $Z_{\mathfrak{s}}$ the submanifold $\Phi^{-1}(a) \cap M_{\mathfrak{s}}$. In order to make use of Theorem 12.18 we need to compute the action of $S$ on the fibers of the line bundle $\left.L\right|_{Z_{\mathfrak{s}}} \otimes \mathbb{C}_{-\mu}$.

Lemma 12.20 If a is a quasi-regular value close enough to $\mu$, the group $S$ acts trivially on the fibers of the line bundle $\left.L\right|_{Z_{\mathfrak{s}}} \otimes \mathbb{C}_{-\mu}$. 
Proof. We consider the $K_{\mu}$-invariant subset $U \subset M$ formed by the points $m \in M$ such that the algebra $\mathfrak{k}_{m} \cap \mathfrak{k}_{\mu}$ acts trivially on $\left.L\right|_{m} \otimes \mathbb{C}_{-\mu}$. The Kostant formulas shows that $\Phi^{-1}(\mu) \subset U$, and if we use local symplectic models of orbits $K_{\mu} \cdot m$, one sees that $U$ is an open subset. Hence $\Phi^{-1}(a) \subset U$ for $a$ close enough to $\mu$. The proof is completed.

Finally, we can use Theorem 12.18 when $a$ is a quasi-regular value close enough to $\mu$ : relation (12.39) gives

$$
\mathrm{m}_{\mu}(L)=\left[R R_{K}\left(M \times(K a)^{-}, \mathbb{L}(a), \Phi_{a}^{-1}(0), \Phi_{a}\right)\right]^{K}=R R\left(M_{a}, L_{\mu, a}\right) .
$$

The proof of Theorem 12.3 is then completed.

\subsection{Quasi polynomial behavior}

We work in the same setting than in Subsection 12.4. We fix a $\Phi$-moment line bundle $L$ on $M$. For any dominant weight $\mu \in \Lambda_{\geq 0}$ and any integer $k \in \mathbb{N}$, we define $\mathrm{m}(k, \mu) \in \mathbb{N}$ as the multiplicity of the representation $V_{\mu}^{K}$ in $R R_{K}\left(M, L^{\otimes k}\right)$.

We introduce

1. the vector subspace $V_{M} \subset \mathbb{R} \oplus \mathfrak{t}^{*}$ generated by $\{1\} \times \Delta(M)$,

2. the cone $C_{M}=\mathbb{R}_{\geq 0}(\{1\} \times \Delta(M))$ contained in $V_{M}$,

3. the lattice $\Lambda_{M}=\{\mathbb{Z} \oplus \Lambda\} \cap V_{M}$,

4. the principal face $\sigma$ for the $K$-manifold : $\sigma$ is the relative interior of a face of the Weyl chamber and $\sigma \cap \Delta(M)$ is dense in $\Delta(M)$.

Theorem 12.3 tells us that $\mathrm{m}(k, \mu)=0$ if $(k, \mu) \notin C_{M}$. We will now give some qualitative properties on the behavior of the multiplicities $\mathrm{m}(k, \mu)$ on the cone $C_{M}$.

If $\xi$ varies in $\sigma$, the subgroup $K_{\xi}$ is independent of $\xi$. We denote it by $K_{\sigma}$. Let $Y=\Phi^{-1}(\sigma)$ be the slice attached to the principal face. It is a $K_{\sigma}$-invariant symplectic submanifold of $M$, and the subgroup $\left[K_{\sigma}, K_{\sigma}\right]$ acts trivially on $\left.L\right|_{Y} \rightarrow Y$ [23]. The vector space $\mathbb{R} \sigma$ is naturally identified with the dual of the Lie algebra of the torus $A_{\sigma}:=K_{\sigma} /\left[K_{\sigma}, K_{\sigma}\right]$. Hence the restriction $\Phi_{Y}=\left.\Phi\right|_{Y}: Y \rightarrow \sigma$ corresponds to the moment map relative to the action of $A_{\sigma}$ on $\left(Y,\left.\Omega\right|_{Y}\right)$.

Let $I_{M} \subset \mathbb{R} \sigma$ be the affine subspace generated by $\Delta(M)$ : the orthogonal, denoted $\mathfrak{t}_{M}$, of the direction of $I_{M}$ in $\operatorname{Lie}\left(A_{\sigma}\right)$ corresponds to the generic infinitesimal stabilizer for the $A_{\sigma}$-action on $Y$. Let $\Delta(M)^{0} \subset \Delta(M) \cap \sigma$ be 
the open subset formed by the regular values of $\Phi_{Y}: Y \rightarrow I_{M}$. Note that any element $\xi \in \Delta(M)^{0}$ is a quasi-regular value of $\Phi$. The reduced space $M_{\xi}$ stays of constant dimension when $\xi \in \Delta(M)^{0}$, and this dimension is the maximal dimension of the reduced spaces $M_{\xi}$, where $\xi$ varies over the set of quasi-regular values of $\Phi$.

If $\mathfrak{a} \subset \Delta(M)^{0}$ is a connected component, we choose a point $\xi \in \mathfrak{a}$, and we consider

- the closed subcone $C_{\mathfrak{a}}=\mathbb{R}_{\geq 0}(\{1\} \times \overline{\mathfrak{a}})$ of $C_{M}$,

- the symplectic orbifold $M_{\mathfrak{a}}:=\Phi^{-1}(\xi) / A_{\sigma}$,

- the family of line orbi-bundle $L_{\mathfrak{a}}^{k, \mu} \rightarrow M_{\mathfrak{a}}$ parameterized by $(k, \mu) \in$ $\Lambda_{M}$ :

$$
L_{\mathfrak{a}}^{k, \mu}:=\left(\left.L^{\otimes k}\right|_{\Phi^{-1}(\xi)} \otimes \mathbb{C}_{-\mu}\right) / A_{\sigma}
$$

The line orbi-bundles $L_{\mathfrak{a}}^{k, \mu}$ are well-defined for any $(k, \mu) \in \Lambda_{M}$ since $\mathcal{L}(X)=0$ on $\left.L^{\otimes k}\right|_{\Phi^{-1}(\xi)} \otimes \mathbb{C}_{-\mu}$ for any $X \in \mathfrak{t}_{M}$.

Definition 12.21 Let $\mathfrak{a}$ be a connected component of $\Delta(M)^{0}$. We consider the function $p_{\mathfrak{a}}: \Lambda_{M} \rightarrow \mathbb{Z}$ defined by the relation

$$
p_{\mathfrak{a}}(k, \mu):=R R\left(M_{\mathfrak{a}}, L_{\mathfrak{a}}^{k, \mu}\right)
$$

The Kawasaki-Riemann-Roch Theorem [19] implies that the function $p_{\mathfrak{a}}$ have a quasi polynomial behavior on the lattice $\Lambda_{M}$.

The function $p_{\mathfrak{a}}$ does not depend on the choice of the choice of $\xi$ in $\mathfrak{a}$. It is due to the fact that $p_{\mathfrak{a}}(k, \mu)$ is equal to

$$
\left[R R_{A_{\sigma}}\left(Y,\left.L^{\otimes k}\right|_{Y}, \Phi_{Y}^{-1}(\xi), \Phi_{Y}-\xi\right) \otimes \mathbb{C}_{-\mu}\right]^{A_{\sigma}} .
$$

See (12.39). Hence if we take another $\xi^{\prime} \in \mathfrak{a}$, we consider a continuous family $\xi_{t} \in \mathfrak{a}, t \in[0,1]$ joining $\xi$ and $\xi^{\prime}$. By an easy homotopy argument, we see that $\left[R R_{A_{\sigma}}\left(Y,\left.L^{\otimes k}\right|_{Y}, \Phi_{Y}^{-1}\left(\xi_{t}\right), \Phi_{Y}-\xi_{t}\right) \otimes \mathbb{C}_{-\mu}\right]^{A_{\sigma}}$ is independent of $t \in[0,1]$.

We can now state the main result of this section. Notice that $C_{M}=$ $\bigcup_{\mathfrak{a}} C_{\mathfrak{a}}$.

Theorem 12.22 If $(k, \mu) \in C_{\mathfrak{a}}$, we have $\mathrm{m}(k, \mu)=p_{\mathfrak{a}}(k, \mu)$. 
Proof. Let us fix $(k, \mu) \in C_{\mathfrak{a}}$. We apply Theorem 12.3 to the $K$ manifold $\mathbb{X}:=(M, k \Omega, k \Phi)$ equipped with the moment line bundle $\mathbb{L}:=L^{\otimes k}$. The multiplicity of $V_{\mu}^{K}$ in $R R_{K}(\mathbb{X}, \mathbb{L})$ is equal to $R R\left(\mathbb{K}_{a}, \mathbb{L}_{a, \mu}\right)$ where $a$ is any quasi-regular value of $k \Phi$ close enough to $\mu$. We can take $a=k \xi$ with $\xi \in \mathfrak{a}$ : the reduced space $\mathbb{X}_{a}$ is equal to $M_{\xi}$ and the line bundle $\mathbb{L}_{a, \mu}$ coincides with $L_{\mathfrak{a}}^{k, \mu}$. We have proved that $\mathrm{m}(k, \mu)=R R\left(\mathbb{K}_{a}, \mathbb{L}_{a, \mu}\right)=p_{\mathfrak{a}}(k, \mu)$.

\subsection{Multiplicities on a face}

Let $F$ be a (closed) face of the polytope $\Delta(M)$ that intersects the principal face $\sigma$. We say that $F$ is a general face of $\Delta(M)$.

We assume in this section that $\mu$ is a dominant weight that belongs to $F$. Let $T_{\sigma} \subset T$ be the connected component of the center of the subgroup $K_{\sigma}$. The dual of the Lie algebra of $T_{\sigma}$ is the subspace $\mathbb{R} \sigma \subset \mathfrak{t}^{*}$. Let $T_{F} \subset T_{\sigma}$ be the subtorus with Lie algebra equal to the orthogonal of the direction $\vec{F} \subset \mathbb{R} \sigma$ of the face $F$. We denote $K_{F}$ the subgroup of $K$ that centralizes $T_{F}$ : we see that $K_{\sigma} \subset K_{F}$.

We start with the basic fact.

Lemma 12.23 The pullback $\Phi^{-1}(F \cap \sigma)$ is connected and belongs to the submanifold $M^{T_{F}}$.

Proof. The pullback $\Phi^{-1}(F \cap \sigma)$ belongs to the slice $Y=\Phi^{-1}(\sigma)$, and it is connected as each fiber $\Phi^{-1}(\xi)$ is connected. Since $F$ is a face of the polytope $\Delta(M) \subset \mathbb{R} \sigma$, we see that $\Delta(M)$ is contained in a cone of the form

$$
\bigcap_{j=1}^{p}\left\{\xi \in \mathbb{R} \sigma \mid\left\langle\xi, X_{j}\right\rangle \geq\left\langle\mu, X_{j}\right\rangle\right\}
$$

where $X_{1}, \ldots, X_{p}$ is a basis of $\operatorname{Lie}\left(T_{F}\right)$. Since $\Delta(M) \cap \sigma=\operatorname{Image}\left(\left.\Phi\right|_{Y}\right)$, the functions $y \in Y \mapsto\left\langle\Phi(y), X_{j}\right\rangle$ reach their minimum at any point of $\Phi^{-1}(F \cap \sigma)$. So the differential of the function $y \in Y \mapsto\left\langle\Phi(y), X_{j}\right\rangle$ vanishes at any point of $\Phi^{-1}(F \cap \sigma)$. This implies that $\Phi^{-1}(F \cap \sigma) \subset \bigcap_{j=1}^{p} M^{X_{j}}=$ $M^{T_{F}}$.

Let $M_{F}$ be the connected component of $M^{T_{F}}$ that contains $\Phi^{-1}(F \cap \sigma)$. It is a $K_{F}$-Hamiltonian manifold equipped with the two form $\Omega_{F}:=\left.\Omega\right|_{M_{F}}$ and the moment map $\Phi_{F}=\left.\Phi\right|_{M_{F}}: M_{F} \rightarrow \mathfrak{k}_{F}^{*}$. We denote $\left.L\right|_{F}$ the restriction of the $\Phi$-moment line bundle $L$ on $M_{F}$. It is easy to see that $\left.L\right|_{F}$ is a $\Phi_{F^{-}}$ moment line bundle. 
We parameterize the irreducible representations of $K_{F}$ thanks to the choice of a Weyl chamber $\mathcal{C}_{F} \subset \mathfrak{t}^{*}$ that contains the Weyl chamber $\mathfrak{t}_{>0}^{*}$ of $K$. So, we may consider the irreducible representation $V_{\mu}^{K_{F}}$ of the group $K_{F}$ with highest weight $\mu$.

A direct consequence of the $[Q, R]=0$ Theorem is the following proposition.

Proposition 12.24 Let $L$ be a $\Phi$-moment bundle on $M$, and let $F$ be a face of $\Delta(M)$ that intersects the principal face $\sigma$. For any dominant weight $\mu$ that belongs to $F$, the multiplicity $\mathrm{m}_{\mu}(L)$ of the representation $V_{\mu}^{K}$ in $R R_{K}(M, L)$ is equal to the multiplicity of the representation $V_{\mu}^{K_{F}}$ in $R R_{K_{F}}\left(M_{F},\left.L\right|_{F}\right)$.

Proof. We have $\mathrm{m}_{\mu}(L)=R R\left(M_{a}, L_{a, \mu}\right)$ for any quasi-regular value of $\Phi$ close enough to $\mu$. Since $\mu \in F$, we can choose $a$ in $F \cap \sigma$, and then $\Phi^{-1}(a)=\Phi_{F}^{-1}(a) \subset M_{F}$. The proposition follows then from the fact that the datas $L_{a, \mu} \rightarrow M_{a}$ and $\left(\left.L\right|_{F}\right)_{a, \mu} \rightarrow\left(M_{F}\right)_{a}$ are equal (the last one is a reduction relatively to the action of the group $K_{F}$ on $\left.\left.L\right|_{F} \rightarrow M_{F}\right)$.

\section{Branching laws}

Let $\tilde{K}$ be a compact connected Lie group and let $K$ be a closed connected subgroup. Let $i$ denote the inclusion of $K$ into $\tilde{K}, i: \mathfrak{k} \rightarrow \tilde{\mathfrak{k}}$ the induced embedding of Lie algebras, and $\pi: \tilde{\mathfrak{k}}^{*} \rightarrow \mathfrak{k}^{*}$ the dual projection.

We consider the following action of $\tilde{K} \times K$ on $\tilde{K}:(\tilde{k}, k) \cdot g=\tilde{k} g k^{-1}$. Then $N=\mathrm{T}^{*} \tilde{K}$, the cotangent bundle of $\tilde{K}$ is a $\tilde{K} \times K$ Hamiltonian manifold. Recall from Subsection 8.5 (Equation 8.30) that the corresponding moment map relative to the $\tilde{K} \times K$-action is the map $\Phi=\Phi_{\tilde{K}} \oplus \Phi_{K}: \mathrm{T}^{*} \tilde{K} \rightarrow \tilde{\mathfrak{k}}^{*} \oplus \mathfrak{k}^{*}$ defined by

$$
\Phi_{\tilde{K}}(g, \xi)=-\xi, \quad \Phi_{K}(g, \xi)=\pi\left(g^{-1} \xi\right) .
$$

Select maximal tori $T$ in $K$ and $\tilde{T}$ in $\tilde{K}$ such that $T \subset \tilde{T}$, and Weyl chambers $\tilde{\mathfrak{t}}_{>0}^{*}$ in $\tilde{\mathfrak{t}}^{*}$ and $\mathfrak{t}_{>0}^{*}$ in $\mathfrak{t}^{*}$, where $\mathfrak{t}$ and $\tilde{\mathfrak{t}}$ denote the Lie algebras of $T$, resp. $\tilde{T}$.

Let $\Delta\left(\mathrm{T}^{*} \tilde{K}\right) \subset \tilde{\mathfrak{t}}_{\geq 0}^{*} \times \mathfrak{t}_{\geq 0}^{*}$ be the Kirwan polytope associated to $\Phi$ :

$$
\Delta\left(\mathrm{T}^{*} \tilde{K}\right)=\left\{(\alpha, \beta) \in \tilde{\mathfrak{t}}_{\geq 0}^{*} \times \mathfrak{t}_{\geq 0}^{*} \mid-\beta \in \pi(\tilde{K} \alpha)\right\} .
$$

For $(\tilde{\lambda}, \lambda) \in \tilde{\mathfrak{t}}_{\geq 0}^{*} \oplus \mathfrak{t}_{\geq 0}^{*}$, we denote by $N_{\tilde{\lambda}, \lambda}$ the reduced space of $N:=\mathrm{T}^{*} \tilde{K}$ at $(\tilde{\lambda}, \lambda)$. Consider the coadjoint orbit $\tilde{K} \tilde{\lambda}^{*}:=-\tilde{K} \tilde{\lambda}$, equipped with the $K$ action and moment map $\left.\pi\right|_{\tilde{K} \tilde{\lambda}^{*}}$. Let $\left(\tilde{K} \tilde{\lambda}^{*}\right)_{\lambda}$ the reduced manifold of $\tilde{K} \tilde{\lambda}^{*}$ at $\lambda$. The following lemma is obvious. 
Lemma 13.1 As sets, $\left(\tilde{K} \tilde{\lambda}^{*}\right)_{\lambda}$ is isomorphic to $N_{\tilde{\lambda}, \lambda}$.

Let $\tilde{\Lambda}_{\geq 0} \subset \tilde{\mathfrak{t}}_{\geq 0}^{*}, \Lambda_{\geq 0} \subset \mathfrak{t}_{\geq 0}^{*}$ be the set of dominant weights. For any $(\lambda, \tilde{\lambda}) \in \Lambda_{\geq 0} \times \tilde{\Lambda}_{\geq 0}$, we denote $V_{\lambda}, \tilde{V}_{\tilde{\lambda}}$ the corresponding irreducible representations of $K$ and $\tilde{K}$, and we define

$$
\mathrm{m}(\tilde{\lambda}, \lambda) \in \mathbb{N}
$$

as the dimension of the vector space $\left[\left.\tilde{V}_{\tilde{\lambda}}\right|_{K} \otimes V_{\lambda}\right]^{K}$. In other words $\mathrm{m}(\tilde{\lambda}, \lambda)$ is the multiplicity of the representation $V_{\lambda}$ in $\left.\left(\tilde{V}_{\tilde{\lambda}}\right)^{*}\right|_{K}$.

We consider the coadjoint orbit $\tilde{K} \tilde{\lambda}^{*}$, equipped with the prequantum line bundle $L\left(\tilde{\lambda}^{*}\right):=\left[\mathbb{C}_{\tilde{\lambda}^{*}}\right]$. The $[Q, R]=0$ theorem 12.3 says that $\mathrm{m}(\tilde{\lambda}, \lambda)=$ $R R\left(\left(\tilde{K} \tilde{\lambda}^{*}\right)_{\lambda}, L\left(\tilde{\lambda}^{*}\right)_{\lambda}\right)$, in particular $\mathrm{m}(\tilde{\lambda}, \lambda)=0$ if $\left(\tilde{K} \tilde{\lambda}^{*}\right)_{\lambda}=\emptyset$.

If we use Lemma 13.1, we see that the cone $\Delta\left(\mathrm{T}^{*} \tilde{K}\right)$ is related to the multiplicity function (13.41) through the following basic result.

Lemma 13.2 We have $\mathrm{m}(\tilde{\lambda}, \lambda)=0$ if $(\tilde{\lambda}, \lambda) \notin \Delta\left(\mathrm{T}^{*} \tilde{K}\right)$.

Let $(\tilde{X}, X)$ be an element of $\tilde{\mathfrak{t}} \times \mathfrak{t}$. In the following lemma, which follows by direct checking, we describe the manifold $N^{(\tilde{X}, X)}$ of zeroes of the vector field $(\tilde{X}, X)$ on $N=\mathrm{T}^{*} \tilde{K}$. We denote $\tilde{K}_{X} \subset \tilde{K}$ the subgroup that fixes $X$. The cotangent bundle $\mathrm{T}^{*} \tilde{K}_{X}$ is then a submanifold of $\mathrm{T}^{*} \tilde{K}$.

Lemma 13.3 - If $\tilde{X}$ is not conjugated in $\tilde{\mathfrak{k}}$ to $X$, then $N^{(\tilde{X}, X)}=\emptyset$

- If $\tilde{X}=a X$ with $a \in \tilde{K}$, then $N^{(\tilde{X}, X)}=a \cdot \mathrm{T}^{*} \tilde{K}_{X}$.

We recall from general principle that the Kirwan polyhedron has non zero interior in $\tilde{\mathfrak{t}}^{*} \times \mathfrak{t}^{*}$ if and only if the generic stabilizer of the $\tilde{K} \times K$-action on $\mathrm{T}^{*} \tilde{K}$ is finite.

To simplify the exposition, we assume from now on that no nonzero ideal of $\mathfrak{k}$ is an ideal of $\tilde{\mathfrak{k}}$. It implies the following

Lemma 13.4 The intersection of $\Delta\left(\mathrm{T}^{*} \tilde{K}\right)$ with $\tilde{\mathfrak{t}}_{>0}^{*} \times \mathfrak{t}_{>0}^{*}$ has a non empty interior, and the generic stabilizer of the $\tilde{K} \times K$-action on $\mathrm{T}^{*} \tilde{K}$ is finite.

Proof. By the preceding computation, we see that the set $N^{1}$ of elements of $N$ having a non zero stabilizer is $N^{1}=\tilde{K} \cdot \cup_{X \in \mathfrak{k}, X \neq 0} \tilde{T}^{*} \tilde{K}_{X}$.

Using conjugation by $K$, we may assume that $X$ is in $\mathfrak{t}$. Thus we obtain $N^{1}=(\tilde{K} \times K) \cdot \cup_{X \in \mathfrak{t}, X \neq 0} \mathrm{~T}^{*} \tilde{K}_{X}$. 
The set of subgroups $\tilde{K}_{X}$ when $X$ varies in $\mathfrak{t}$ is finite. Consider the fiber product $F(X)=(\tilde{K} \times K) \times_{\tilde{K}_{X} \times K_{X}} \mathrm{~T}^{*} \tilde{K}_{X}$. It surjects on $(\tilde{K} \times K) \cdot \mathrm{T}^{*} \tilde{K}_{X}$. Thus it is sufficient to prove that for $X \neq 0$ in $\mathfrak{t}$, and under our hypothesis, the dimension of $F(X)$ is strictly less than $\mathrm{T}^{*} \tilde{K}$.

Computing dimensions, if the dimensions are equal, then $\operatorname{dim}\left(K / K_{X}\right)=$ $\operatorname{dim}\left(\tilde{K} / \tilde{K}_{X}\right)$. So this implies that $K \cdot X=\tilde{K} \cdot X$. The vector space spanned by $K \cdot X$ is then an ideal of $\tilde{\mathfrak{k}}$ contained in $\mathfrak{k}$, contradicting our hypothesis.

In the next section, we will give some qualitative information concerning the behavior of the multiplicity function $\mathrm{m}(\tilde{\lambda}, \lambda)$ on the cone $\Delta\left(\mathrm{T}^{*} \tilde{K}\right)$.

\subsection{Quasi polynomial behavior}

We consider the slice

$$
Y=\Phi^{-1}\left(\tilde{\mathfrak{t}}_{>0}^{*} \times \mathfrak{t}_{>0}^{*}\right)
$$

which is a (non-empty) symplectic submanifold of $\mathrm{T}^{*} \tilde{K}$ equipped with the action of $\tilde{T} \times T$.

We denote $\Phi_{Y}: Y \rightarrow \tilde{\mathfrak{t}}^{*} \times \mathfrak{t}^{*}$ the restriction of the moment map on $Y$. Let $\Delta^{0} \subset \Delta\left(\mathrm{T}^{*} \tilde{K}\right)$ be the open cone formed by the regular values of $\Phi_{Y}: Y \rightarrow \tilde{\mathfrak{t}}^{*} \times \mathfrak{t}^{*}$. If $\mathfrak{a} \subset \Delta^{0}$ is a connected component of $\Delta^{0}$, we choose a point $(\tilde{\xi}, \xi) \in \mathfrak{a}$, and we consider:

- the compact connected symplectic orbifold $N_{\mathfrak{a}}:=\Phi_{Y}^{-1}(\tilde{\xi}, \xi) / \tilde{T} \times T$,

- the family of line orbi-bundle $L_{\mathfrak{a}}^{\tilde{\alpha}, \alpha} \rightarrow N_{\mathfrak{a}}$ parametrized by $(\tilde{\alpha}, \alpha) \in$ $\tilde{\Lambda} \times \Lambda$ :

$$
L_{\mathfrak{a}}^{\tilde{\alpha}, \alpha}:=\Phi_{Y}^{-1}(\tilde{\xi}, \xi) \times_{\tilde{T} \times T} \mathbb{C}_{-\tilde{\alpha},-\alpha} .
$$

Here $\mathbb{C}_{-\tilde{\alpha},-\alpha}$ denotes the one dimensional representation of $\tilde{T} \times T$ where the group acts through the character $\chi_{-\tilde{\alpha},-\alpha}\left(t_{1}, t_{2}\right)=t_{1}^{-\tilde{\alpha}} t_{2}^{-\alpha}$.

Definition 13.5 Let $\mathfrak{a}$ be a connected component of $\Delta\left(\mathrm{T}^{*} K\right)^{0}$. We consider the function $p_{\mathfrak{a}}: \tilde{\Lambda} \times \Lambda \rightarrow \mathbb{Z}$ defined by the relation

$$
p_{\mathfrak{a}}(\tilde{\alpha}, \alpha):=R R\left(N_{\mathfrak{a}}, L_{\mathfrak{a}}^{\tilde{\alpha}, \alpha}\right) .
$$

The function $p_{\mathfrak{a}}$ does not depend on the choice of the choice of $\tilde{\xi}, \xi$ in $\mathfrak{a}$, since the $\tilde{T} \times T$-manifolds $\Phi_{Y}^{-1}\left(\tilde{\xi}_{1}, \xi_{1}\right)$ and $\Phi_{Y}^{-1}\left(\tilde{\xi}_{2}, \xi_{2}\right)$ are diffeomorphic for two different choices. The Kawasaki-Riemann-Roch Theorem [19] implies that the function $p_{\mathfrak{a}}$ have a quasi polynomial behavior on the lattice $\tilde{\Lambda} \times \Lambda$.

We can now state the main result of this section. Note that $\Delta\left(\mathrm{T}^{*} K\right)=$ $\bigcup_{\mathfrak{a}} \overline{\mathfrak{a}}$. 
Theorem 13.6 If $(\tilde{\lambda}, \lambda) \in \overline{\mathfrak{a}}$, we have $\mathrm{m}(\tilde{\lambda}, \lambda)=p_{\mathfrak{a}}(\tilde{\lambda}, \lambda)$.

Proof. Let $b:=(\tilde{\xi}, \xi) \in \mathfrak{a}$ and $(\tilde{\lambda}, \lambda) \in \overline{\mathfrak{a}}$. By (12.38), the quantity $p_{\mathfrak{a}}(\tilde{\lambda}, \lambda)$ is equal to

$$
\left[R R_{\tilde{T} \times T}\left(Y, \mathbb{C}, \Phi_{Y}^{-1}(b), \Phi_{Y}-b\right) \otimes \mathbb{C}_{-\tilde{\lambda},-\lambda}\right]^{\tilde{T} \times T} .
$$

We write $\Phi_{Y}=\Phi_{\tilde{T}} \oplus \Phi_{T}$. An element $(g, \eta)$ belongs to $Y$ if and only if $-\eta \in \tilde{\mathfrak{t}}_{>0}^{*}$ and $\pi\left(g^{-1} \eta\right) \in \mathfrak{t}_{>0}^{*}$. The group $\tilde{T}$ acts freely on $Y$, and the smooth reduced space $Y_{\tilde{\xi}}=\Phi_{\tilde{T}}^{-1}(\tilde{\xi}) / \tilde{T}$ is symplectomorphic to the slice

$$
\left\{\eta \in \tilde{K} \tilde{\xi}^{*} \mid \pi(\eta) \in \mathfrak{t}_{>0}^{*}\right\} \subset \tilde{K} \tilde{\xi}^{*}
$$

through the map $(g, \eta) \rightarrow g^{-1} \eta$. We have

$$
\begin{aligned}
& {\left[R R_{\tilde{T} \times T}\left(Y, \mathbb{C}, \Phi_{Y}^{-1}(b), \Phi_{Y}-b\right) \otimes \mathbb{C}_{-\tilde{\lambda},-\lambda}\right]^{\tilde{T} \times T}} \\
& \quad=\left[R R_{T}\left(Y_{\tilde{\xi}},\left[\mathbb{C}_{-\tilde{\lambda}}\right], \Phi_{T}^{-1}(\xi), \Phi_{T}-\xi\right) \otimes \mathbb{C}_{-\lambda}\right]^{T} \\
& \quad=\left[R R_{K}\left(\tilde{K} \tilde{\xi}^{*} \times(K \xi)^{-},\left[\mathbb{C}_{-\tilde{\lambda}}\right] \otimes\left[\mathbb{C}_{-\lambda}\right], \Phi_{b}^{-1}(0), \Phi_{b}\right)\right]^{K}
\end{aligned}
$$

where $\Phi_{b}: \tilde{K} \tilde{\xi}^{*} \times \overline{K \xi} \rightarrow \mathfrak{k}^{*}$ is the moment map defined by $\Phi_{b}(\tilde{\eta}, \eta)=\pi(\tilde{\eta})-\eta$. Equality (1) follows from the reduction in stage property (see Proposition $12.6)$, and (2) is a particular case of (12.40).

If $b=(\tilde{\xi}, \xi) \in \mathfrak{a}$ is close enough to $(\tilde{\lambda}, \lambda)$, the line bundle $\left[\mathbb{C}_{-\tilde{\lambda}}\right] \otimes\left[\mathbb{C}_{-\lambda}\right]$ is weakly $\Phi_{b}$-positive (see Lemma 12.19). Since $\Phi_{b}^{-1}(0) \neq \emptyset$ we can conclude that

$$
\begin{aligned}
& {\left[R R_{K}\left(\tilde{K} \tilde{\xi}^{*} \times(K \xi)^{-},\left[\mathbb{C}_{-\tilde{\lambda}}\right] \otimes\left[\mathbb{C}_{-\lambda}\right], \Phi_{b}^{-1}(0), \Phi_{b}\right)\right]^{K}} \\
& \quad=\left[R R_{K}\left(\tilde{K} \tilde{\xi}^{*} \times(K \xi)^{-},\left[\mathbb{C}_{-\tilde{\lambda}}\right] \otimes\left[\mathbb{C}_{-\lambda}\right]\right)\right]^{K} \\
& \quad=\left[\left.\left(\tilde{V}_{\tilde{\lambda}}\right)^{*}\right|_{K} \otimes V_{\lambda}^{*}\right]^{K} \\
& \quad=\mathrm{m}(\tilde{\lambda}, \lambda) .
\end{aligned}
$$

Remark 13.7 In the proof above, we obtain the following identity

$$
\mathrm{m}(\tilde{\lambda}, \lambda)=\left[R R_{\tilde{T} \times T}\left(Y, \mathbb{C}, \Phi_{Y}^{-1}(b), \Phi_{Y}-b\right) \otimes \mathbb{C}_{-\tilde{\lambda},-\lambda}\right]^{\tilde{T} \times T},
$$

that holds for any $b=(\tilde{\xi}, \xi) \in \tilde{\mathfrak{t}}_{>0}^{*} \times \mathfrak{t}_{>0}^{*}$ that is close enough to $(\tilde{\lambda}, \lambda)$. The identity $\mathrm{m}(\tilde{\lambda}, \lambda)=p_{\mathfrak{a}}(\tilde{\lambda}, \lambda)$ follows by taking $(\tilde{\xi}, \xi)$ a regular value of $\Phi$. But we could have taken a quasi-regular value of $\Phi$ (see Section 13.2). 
We can also view this theorem as a consequence of $[Q, R]=0$ for the non compact manifold $N=\mathrm{T}^{*} \tilde{K}$. Philosophically, we have used here reduction in stages to reduce to the $\tilde{K} \times K$ reduced space of $N$ to the reduced space with respect to $K$ of the reduced space with respect to $\tilde{K}$ of $N$.

We could however, at the price of proving $[Q, R]=0$ directly for the non compact manifold $N$, prove directly our theorem. Let us sketch the proof. If we use the result of Section 8.5, knowing that the critical set $Z_{\Phi}$ is compact, we see that the localized index $R R_{\tilde{K} \times K}\left(N,[\mathbb{C}], Z_{\Phi}\right)$ is equal to $\left.\sum_{\tilde{\eta}} \tilde{V}_{\tilde{\eta}}^{*} \otimes \tilde{V}_{\tilde{\eta}}\right|_{K}$ in $\hat{R}(\tilde{K} \times K)$.

The quantity $\mathrm{m}(\tilde{\lambda}, \lambda)$ is then the multiplicity of $\tilde{V}_{\tilde{\lambda}} \otimes V_{\lambda}$ in the character $R R_{\tilde{K} \times K}\left(N,[\mathbb{C}], Z_{\Phi}\right)$. Note that here the trivial line bundle $[\mathbb{C}]$ is a $\Phi$-moment line bundle on $N$. By an adaptation of the shifting trick to this non-compact setting one can prove directly that $\mathrm{m}(\tilde{\lambda}, \lambda)=R R\left(N_{\tilde{\lambda}, \lambda},[\mathbb{C}]_{\tilde{\lambda}, \lambda}\right)$. This is the content of Theorem 13.6 since the term $R R\left(N_{\tilde{\lambda}, \lambda},\left[\mathbb{C}_{\tilde{\lambda}, \lambda}\right)\right.$ is equal to $p_{\mathfrak{a}}(\tilde{\lambda}, \lambda)$ for any chamber $\mathfrak{a}$ containing $(\tilde{\lambda}, \lambda)$ in its closure.

\subsection{Multiplicities on a face}

Let us give the structure of the general faces of the cone $\Delta\left(\mathrm{T}^{*} \tilde{K}\right) \subset \tilde{\mathfrak{t}}_{\geq 0}^{*} \times \mathfrak{t}_{\geq 0}^{*}$. Let $F$ be a closed face of $\Delta\left(\mathrm{T}^{*} \tilde{K}\right)$ that intersects $\tilde{\mathfrak{t}}_{>0}^{*} \times \mathfrak{t}_{>0}^{*}$. We say that $F$ is a general face. We denote $\langle F\rangle$ the vector subspace generated by $F$, and $\mathfrak{g}_{F}$ its orthogonal in $\tilde{\mathfrak{t}} \times \mathfrak{t}$. Note that $F=\langle F\rangle \cap \Delta\left(T^{*} \tilde{K}\right)$.

We denote $\tilde{W}$ the Weyl group of $\tilde{K}$ relatively to the maximal torus $\tilde{T}$.

Lemma 13.8 • There exists a vector subspace $\mathfrak{t}_{F} \subset \mathfrak{t}$ and $\tilde{w} \in \tilde{W}$ such that $\mathfrak{g}_{F}=\left\{(\tilde{w} X, X), X \in \mathfrak{t}_{F}\right\}$.

- For any weights $(\tilde{\lambda}, \lambda)$ contained in $F$, the weight $\tilde{w}^{-1} \tilde{\lambda}+\lambda$ vanishes on $\mathfrak{t}_{F}$.

- The connected component of $\left(\mathrm{T}^{*} \tilde{K}\right)^{\mathfrak{g}_{F}}$ that contains $\Phi^{-1}\left(F \cap \tilde{\mathfrak{t}}_{>0}^{*} \times \mathfrak{t}_{>0}^{*}\right)$ is $\tilde{w} \cdot \mathrm{T}^{*} \tilde{K}_{F}$. Here $\tilde{K}_{F}$ is the subgroup of $\tilde{K}$ that centralizes $\mathfrak{t}_{F}$.

Proof. We know that any element $(\tilde{X}, X) \in \mathfrak{g}_{F}$ fixes the points of $\Phi^{-1}\left(F \cap \tilde{\mathfrak{t}}_{>0}^{*} \times \mathfrak{t}_{>0}^{*}\right)$ (see Lemma 12.23), and that forces to have $\tilde{X}=\tau X$ for some $\tau \in \tilde{W}$ (see Lemma 13.3). The first point follows. The second point is obvious and the third follows directly from Lemma 13.3.

Berenstein-Sjamaar [7] and Ressayre [42, 43] have determined, in terms of Schubert calculus, what are the data $\left(\tilde{w}, \mathfrak{t}_{F}\right)$ that comes from a face $F$. It is usually difficult to list them explicitly. 
We denote by $K_{F}$ the subgroup of $K$ that centralizes $\mathfrak{t}_{F}$. The element $\tilde{w}$ is defined modulo the Weyl group of $K_{F}$. We thus can choose $\tilde{w}$ such that for any root $\alpha$ of $\tilde{K}_{F}$, we have $\alpha>0 \Leftrightarrow \tilde{w} \alpha>0$.

The subgroup of $\tilde{K} \times K$ that centralizes $\mathfrak{g}_{F}$ is of the form $\tilde{w} \tilde{K}_{F} \tilde{w}^{-1} \times K_{F}$. So for weights $(\tilde{\lambda}, \lambda)$ contained in $F$, the weight

$$
\widehat{\lambda}:=\tilde{w}^{-1} \tilde{\lambda}
$$

is dominant for $\tilde{K}_{F}$ : we denote $\tilde{V}_{\hat{\lambda}}^{F}$ the corresponding irreducible representation of $\tilde{K}_{F}$. Similarly the weight $\lambda$ is dominant for $K_{F}$ and we denote $V_{\lambda}^{F}$ the corresponding irreducible representation of $K_{F}$.

We end this section with the following result obtained by Ressayre in [44], which is a non-compact analogue of Proposition 12.24.

Proposition 13.9 For any dominant weights $(\tilde{\lambda}, \lambda)$ contained in $F$, the dimension of $\left[\left.\tilde{V}_{\tilde{\lambda}}\right|_{K} \otimes V_{\lambda}\right]^{K}$ is equal to the dimension of $\left[\left.\tilde{V}_{\hat{\lambda}}^{F}\right|_{K_{F}} \otimes V_{\lambda}^{F}\right]^{K_{F}}$.

Proof. We denote $G_{F}$ the subtorus of $\tilde{T} \times T$ with Lie algebra $\mathfrak{g}_{F}$.

We start with the identity

$$
\left[\left.\tilde{V}_{\tilde{\lambda}}\right|_{K} \otimes V_{\lambda}\right]^{K}=\left[R R_{\tilde{T} \times T}\left(Y, \mathbb{C}, \Phi_{Y}^{-1}(b), \Phi_{Y}-b\right) \otimes \mathbb{C}_{-\tilde{\lambda},-\lambda}\right]^{\tilde{T} \times T},
$$

that holds for any $b=(\tilde{\xi}, \xi) \in \tilde{\mathfrak{t}}_{>0}^{*} \times \mathfrak{t}_{>0}^{*}$ that is close enough to $(\tilde{\lambda}, \lambda)$. See Remark 13.7. Let us use it with $(\tilde{\xi}, \xi)$ a generic element contained in $F \cap\left(\tilde{\mathfrak{t}}_{>0}^{*} \times \mathfrak{t}_{>0}^{*}\right)$ and close enough to $(\tilde{\lambda}, \lambda)$ : it is a quasi-regular value of $\Phi$ such that the fiber $\Phi^{-1}(\tilde{\xi}, \xi) \subset \tilde{w} \cdot \mathrm{T}^{*} \tilde{K}_{F}$ is a smooth submanifold with a locally free action of the group $(\tilde{T} \times T) / G_{F}$,

Thanks to Theorem 12.18, we know that the right hand side of (13.42) is equal to $R R\left(N_{\tilde{\xi}, \xi}, \mathbb{L}_{\tilde{\lambda}, \lambda}\right)$ where

$$
\mathbb{Z}_{\tilde{\lambda}, \lambda}=\Phi^{-1}(\tilde{\xi}, \xi) \times{ }_{(\tilde{T} \times T) / G_{F}} \mathbb{C}_{-\tilde{\lambda},-\lambda}
$$

is an line orbibundle on the symplectic orbifold $N_{\tilde{\xi}, \xi}=\Phi^{-1}(\tilde{\xi}, \xi) /((\tilde{T} \times$ $\left.T) / G_{F}\right)$

We consider now the Hamiltonian action of $\tilde{K}_{F} \times K_{F}$ on $N_{F}:=\mathrm{T}^{*} \tilde{K}_{F}$. Let us denote $\Phi_{F}: N_{F} \rightarrow \tilde{\mathfrak{k}}_{F}^{*} \times \mathfrak{k}_{F}^{*}$ the corresponding moment map. The torus $T_{F}$ with Lie algebra $\mathfrak{t}_{F}$ is embedded diagonaly in $\tilde{T} \times T$. For a generic element $(\alpha, \beta) \in \Delta\left(N_{F}\right)$, the fiber $\Phi_{F}^{-1}(\alpha, \beta)$ is a smooth submanifold with a locally free action of $(\tilde{T} \times T) / T_{F}$. 
As in the previous setting, we know that the multiplicity $\left[\left.\tilde{V}_{\tilde{\lambda}}^{F}\right|_{K_{F}} \otimes V_{\lambda}^{F}\right]^{K_{F}}$ is equal to $R R\left(\left(N_{F}\right)_{\widehat{\xi}, \xi}, \mathbb{L}_{\widehat{\lambda}, \lambda}\right)$ when $(\widehat{\xi}, \xi) \in \Delta\left(N_{F}\right)$ is a generic element close enough to $(\widehat{\lambda}, \lambda)$. Here

$$
\mathbb{\complement}_{\widehat{\lambda}, \lambda}=\Phi_{F}^{-1}(\widehat{\xi}, \xi) \times_{(\tilde{T} \times T) / T_{F}} \mathbb{C}_{-\widehat{\lambda},-\lambda}
$$

is an line orbibundle on the symplectic orbifold $\left(N_{F}\right)_{\widehat{\xi}, \xi}=\Phi_{F}^{-1}(\widehat{\xi}, \xi) /((\tilde{T} \times$ $\left.T) / T_{F}\right)$.

Now our proposition follows from the fact that the Riemann-Roch numbers $R R\left(N_{\tilde{\xi}, \xi}, \mathbb{L}_{\tilde{\lambda}, \lambda}\right)$ and $R R\left(\left(N_{F}\right)_{\widehat{\xi}, \xi}, \mathbb{L}_{\widehat{\lambda}, \lambda}\right)$ are equal. It is due to the fact that the multiplication by $\tilde{w}^{-1}$ induces a symplectomorphism $N_{\tilde{\xi}, \xi} \simeq\left(N_{F}\right)_{\widehat{\xi}, \xi}$ and an isomorphism of line bundles $\mathbb{L}_{\tilde{\lambda}, \lambda} \simeq \mathbb{L}_{\widehat{\lambda}, \lambda}$.

\section{References}

[1] M.F. AтזYAн, Elliptic operators and compact groups, Lecture Notes in Mathematics 401, Springer-Verlag, Berlin, 1974.

[2] M.F. AтזYAh, Convexity and commuting Hamiltonians, Bull. London Math. Soc. 14 (1982), 1-15.

[3] M.F. Atiyah and G.B. Segal, The index of elliptic operators II, Ann. of Math. 87 (1968), 531-545.

[4] M.F. Atiyah and I.M. Singer, The index of elliptic operators I, Ann. of Math. 87 (1968), 484-530.

[5] M.F. Atiyah and I.M. Singer, The index of elliptic operators III, Ann. of Math. 87 (1968), 546-604.

[6] M.F. Atiyah and I.M. Singer, The index of elliptic operators IV, Ann. of Math. 93 (1971), 139-141.

[7] A. Berenstein and R. Sjamaar, Coadjoint orbits, moment polytopes, and the Hilbert-Mumford criterion, Journal of the A. M. S. 13 (2000), 433-466.

[8] N. Berline, E. Getzler and M. Vergne, Heat kernels and Dirac operators, Grundlehren 298, Springer, Berlin, 1991.

[9] N. Berline and M. Vergne, The Chern character of a transversally elliptic symbol and the equivariant index, Invent. Math. 124 (1996), 11-49.

[10] N. Berline and M. Vergne, L'indice équivariant des opérateurs transversalement elliptiques, Invent. Math. 124 (1996), 51-101.

[11] M. Braverman, Index theorem for equivariant Dirac operators on noncompact manifolds, K-Theory 27 (2002), 61-101. 
[12] M. BRAVERMAN, The index theory on non-compact manifolds with proper group action, preprint arXiv:1403.7587 (2014).

[13] M. BRION, Restriction de représentations et projections d'orbites coadjointes (d'après Belkale, Kumar et Ressayre), Séminaire Bourbaki, vol. 2011/2012, Astérisque 352 (2013), 1-33.

[14] H. Derksen and J. Weyman, On the Littlewood-Richarson polynomials, J. Algebra 255 (2002), 247-257.

[15] V. Guillemin and S. Sternberg, Convexity properties of the moment mapping, Invent. Math. 67 (1982), 491-513.

[16] V. Guillemin and S. Sternberg, Geometric quantization and multiplicities of group representations, Invent. Math. 67 (1982), 515-538.

[17] V. Guillemin and S. Sternberg, A normal form for the moment map, Differential geometric methods in mathematical physics 6 (1984), 161-175.

[18] T. Kahle and M. Michalek, Plethysm and lattice point counting, arXiv preprint arXiv 14008.5708 (2014).

[19] T. KaWASAKI, The index of elliptic operators over V-manifolds, Nagoya Math. Journal 84 (1981), 135-157.

[20] R.C. King, C. Tollu and F. Toumazet, Factorization of LittlewoodRichardson coefficients, J. of Combinatorial Theory 116 (2009), 314-333.

[21] F.C. KiRwan, Convexity properties of the moment mapping III, Invent. Math. 77 (1984), 547-552.

[22] S. Kumar and D. Prasad, Dimension of zero weight space: An algebrogeometric approach, Journal of Algebra 403 (2014), 324-344.

[23] E. Lerman, E. Meinrenken, S. Tolman and C. Woodward, Non-Abelian convexity by symplectic cuts, Topology 37 (1998), 245-259.

[24] X. MA and W. ZhANG, Geometric quantization for proper moment maps: the Vergne conjecture, Acta Mathematica 212 (2014), 11-57.

[25] P. Hochs and V. Mathai, Quantising proper actions on Spin ${ }^{c}$-manifolds, preprint arXiv:1408.0085 (2014).

[26] V. Mathai and W. Zhang, Geometric quantization for proper actions, Advances in Mathematics 225 (2010), 1224-1247.

[27] E. Meinrenken, On Riemann-Roch formulas for multiplicities, J. Amer. Math. Soc. 9 (1996), 373-389.

[28] E. Meinrenken, Symplectic surgery and the Spin ${ }^{c}$-Dirac operator, Advances in Math. 134 (1998), 240-277.

[29] E. Meinrenken and R. SjamaAR, Singular reduction and quantization, Topology 38 (1999), 699-762. 
[30] K. Mulmuley, Geometric Complexity Theory VI: the flip via saturated and positive integer programming in representation theory and algebraic geometry, preprint arXiv:0704.0229 (2007).

[31] P-E. Paradan, Formules de localisation en cohomologie équivariante, Compositio Mathematica 117 (1999), 243-293.

[32] P-E. Paradan, Localization of the Riemann-Roch character, J. Functional Analysis 187 (2001), 442-509.

[33] P-E. Paradan, Spin ${ }^{c}$ quantization and the K-multiplicities of the discrete series, Annales Scientifiques de l'E. N. S. 36 (2003), 805-845.

[34] P-E. Paradan, Formal geometric quantization, Ann. Inst. Fourier 59 (2009), 199-238.

[35] P.-E. Paradan, Formal geometric quantization II, Pacific J. Math. 253 (2011), 169-211.

[36] P-E. Paradan, Spin quantization commutes with reduction, J. of Symplectic Geometry 10 (2012), 389-422.

[37] P-E. Paradan, Stability property of multiplicities of group representations, preprint arXiv:1510.05080 (2015).

[38] P-E. PAradan and M. Vergne, Index of transversally elliptic operators, Astérique 328 (2009), 297-338.

[39] P-E. Paradan and M. Vergne, The multiplicities of the equivariant index of twisted Dirac operators, C. R. A. S., Volume 352, Issue 9, (2014), 673-677.

[40] P-E. Paradan and M. Vergne, Equivariant Dirac operators and differentiable geometric invariant theory, preprint arXiv:1411.7772 (2014).

[41] P-E. Paradan and M. Vergne, Admissible coadjoint orbits for compact Lie groups, preprint arXiv:1512.02367 (2015).

[42] N. Ressayre, Geometric invariant theory and the generalized eigenvalue problem, Invent. math. 180 (2010), 389-441.

[43] N. Ressayre, Geometric invariant theory and the generalized eigenvalue problem II, Annales Inst. Fourier 61 (2011), 1467-1491.

[44] N. Ressayre, Reductions for branching coefficients, preprint arXiv:1102.0196 (2011).

[45] Y. Song, A K-homological approach to the quantization commutes with reduction problem, preprint arXiv:1404.4840 (2014).

[46] C. Teleman, The quantization conjecture revisited, Annals of Math. 152 (2000), 1-43.

[47] Y. Tian and W. ZHANG, An analytic proof of the geometric quantization conjecture of Guillemin-Sternberg, Invent. Math. 132 (1998), 229-259. 
[48] M. Vergne, Multiplicity formula for geometric quantization, Part I, Part II, and Part III, Duke Math. Journal 82 (1996), 143-179, 181-194, 637-652.

[49] M. Vergne, Quantification géométrique et réduction symplectique, Séminaire Bourbaki, vol. 2000/2001. Astérisque 282 (2002), Exp. No 888, 249-278.

[50] E. Witten, Two dimensional gauge theories revisited, J. Geom. Phys. 9 (1992), 303-368. 